\title{
Secondary Voltage Control using Singular Value Decomposition by Discovering Community Structures in Power Networks
}

Talha Iqbal

Follow this and additional works at: https://researchrepository.wvu.edu/etd

\section{Recommended Citation}

Iqbal, Talha, "Secondary Voltage Control using Singular Value Decomposition by Discovering Community Structures in Power Networks" (2017). Graduate Theses, Dissertations, and Problem Reports. 5858. https://researchrepository.wvu.edu/etd/5858

This Thesis is protected by copyright and/or related rights. It has been brought to you by the The Research Repository @ WVU with permission from the rights-holder(s). You are free to use this Thesis in any way that is permitted by the copyright and related rights legislation that applies to your use. For other uses you must obtain permission from the rights-holder(s) directly, unless additional rights are indicated by a Creative Commons license in the record and/ or on the work itself. This Thesis has been accepted for inclusion in WVU Graduate Theses, Dissertations, and Problem Reports collection by an authorized administrator of The Research Repository @ WVU. For more information, please contact researchrepository@mail.wvu.edu. 


\title{
Secondary Voltage Control using Singular Value Decomposition by Discovering Community Structures in Power Networks
}

\author{
Talha Iqbal \\ Thesis submitted to the \\ College of Engineering and Mineral Resources \\ at West Virginia University \\ in partial fulfillment of the requirements \\ for the degree of \\ Master of Science \\ in \\ Electrical Engineering \\ Ali Feliachi, Ph.D., Chair \\ Muhammad A. Choudhry, Ph.D. \\ Natalia A. Schmid, Ph.D.
}

Lane Department of Computer Science and Electrical Engineering

Morgantown, West Virginia

2017

Keywords:

Secondary Voltage Control, Singular Value Decomposition, Decentralized Control, Network Partitioning, Community Detection 


\title{
ABSTRACT \\ Secondary Voltage Control using Singular Value Decomposition by Discovering Community Structures in Power Networks
}

\author{
Talha Iqbal
}

Voltage and Frequency control are the two fundamental control problems in power systems. Unlike frequency control, voltage control is complicated by the fact that reactive power can't travel far distances from its source of generation. Due to this distributed nature of reactive power, voltage control is usually performed in decentralized manner. Typically, voltage control problem is divided into a three-level hierarchical structure namely primary, secondary and tertiary voltage control.

The aim of this thesis is to present an optimal secondary voltage control by decomposing a large power system into small subsystems called voltage control areas (VCAs) using the fast community detection algorithm. Each VCA is self-sufficient in satisfying its reactive power demand. A load bus, called pilot point/bus, is selected in each VCA as a representative of the voltage profile of the whole area. Singular value decomposition of Fast Decoupled Load Flow (FDLF) Jacobian is used to optimally control the voltages of these pilot buses.

The presented approach is tested on two standard IEEE test power systems i.e. 9-Bus and 39-Bus systems. The computational time comparison of the fast community detection algorithm with another algorithm called original-GN algorithm is also presented. Through simulation results, it is shown that the presented optimal voltage control $(O p t-V C)$ is a better approach compared to sensitivity based voltage control (Sen-VC). 


\section{Acknowledgements}

I would like to express my gratitude to Professor Ali Feliachi, my advisor, for his guidance throughout this research work and many other courses that I have taken at WVU. Without his help and support, I could not finish my MS degree and thesis.

I would also like to thank Professor Muhammad Choudhry and Professor Natalia A. Schmid for serving on my examining committee. The knowledge that I have learned from their classes has helped me a lot in my thesis work.

I would like to extend my appreciation to all my friends who have been with me, supported me and shared a great time since I stepped into WVU.

Finally, I want to give my deepest gratitude to my parents and my wife, Shafia, for the unconditional love and support and especially to my brother, Hasan, who not only guided me but also helped me in pursuing my degree in the United States. 


\section{TABLE OF CONTENTS}

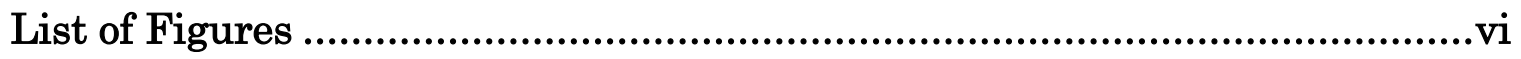

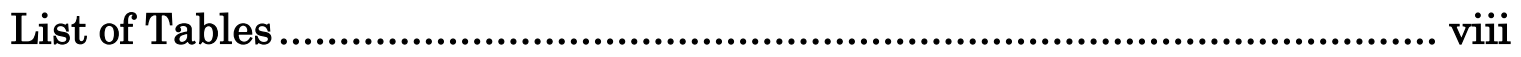

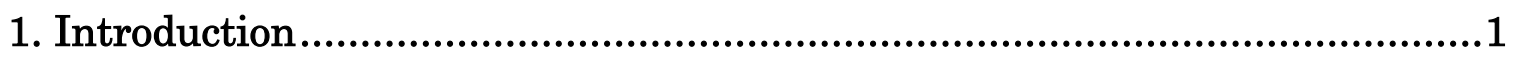

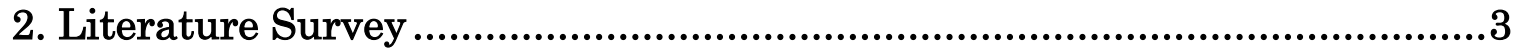

2.1 Identification of Voltage Control Areas (VCAs): .................................... 5

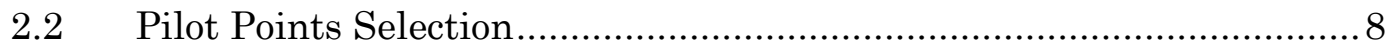

2.3 Control of Pilot Points Voltages ................................................... 8

3. Voltage Control Areas and Pilot Buses ..................................................10

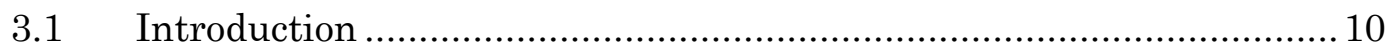

$3.2 \quad$ Optimal Network Partitioning ......................................................... 11

3.2.1 Modularity (a quantification of the quality of a partition) ....................11

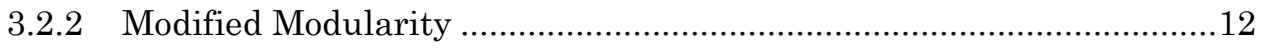

3.2.3 Optimal Network Partitioning Algorithm ..............................................15

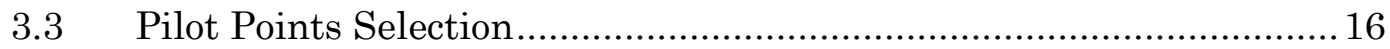

3.3.1 Pilot Bus Selection using Electrical Distances .......................................17

3.4 Case Studies and Simulation Results ............................................... 17

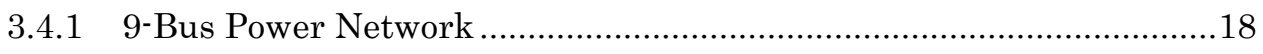

3.4.2 39-Bus Power Network ……………………………………………....21

3.4.3 Performance Comparison ...................................................................26

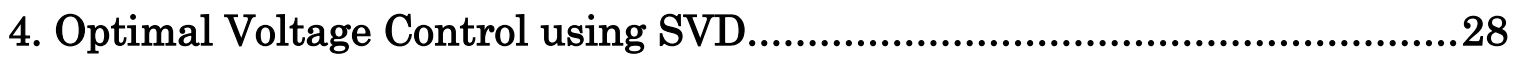

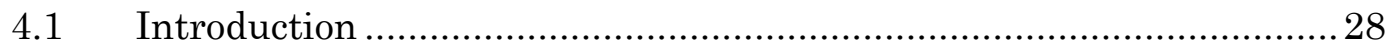

4.2 Problem Formulation.................................................................29

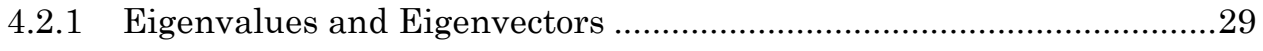

4.2.2 Singular Value Decomposition .............................................................

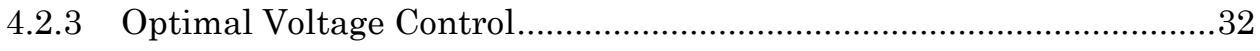

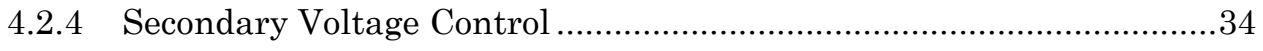

4.3 Case Studies and Simulation Results .................................................. 36 


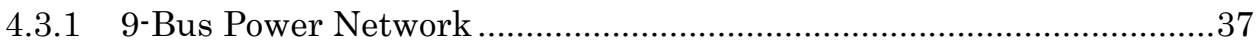

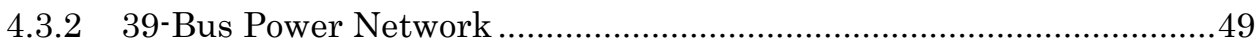

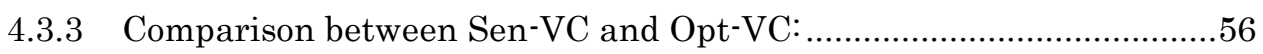

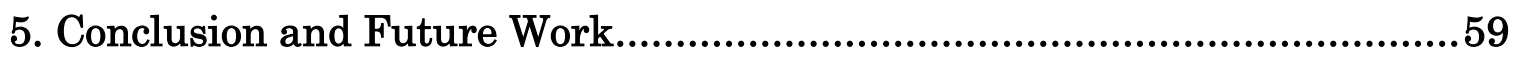

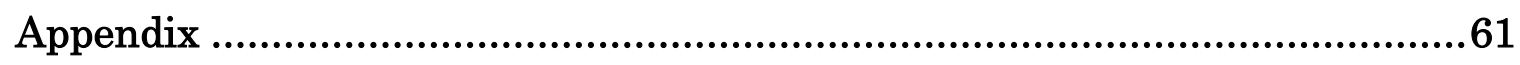

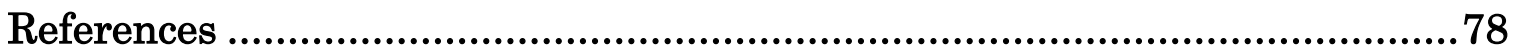




\section{List of Figures}

Figure 2.1: Time Space Delineation of a Three Layer Hierarchical Voltage Control Scheme ...........4

Figure 2.2: Flowchart of Network Partitioning using Electrical Distance ......................................5

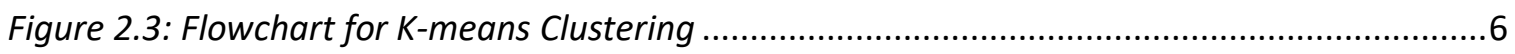

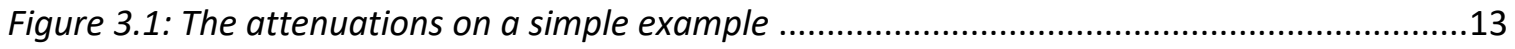

Figure 3.2: Optimal Network Partitioning Algorithm Flowchart ................................................16

Figure 3.3: Electrical Distance Matrix [D $\left.D_{i j}\right]$ (9-Bus Power System) .............................................18

Figure 3.4: Buses in each Community after Optimal Partitioning (9-Bus Power System) ..............19

Figure 3.5: Dendrogram and $M_{\text {mod }}$ variations (9-Bus Power System) ............................................20

Figure 3.6: Selected Pilot Buses in three VCAs (9-Bus Power System) .........................................21

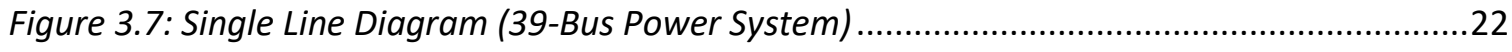

Figure 3.8: Electrical Distance Matrix [D $\left.D_{i j}\right]$ (39-Bus Power Network) .........................................23

Figure 3.9: Dendrogram and $M_{\text {mod }}$ variations (39-Bus Power System)........................................24

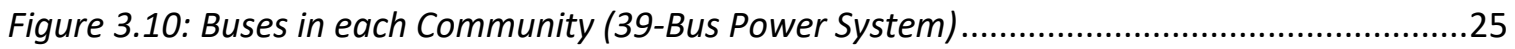

Figure 3.11: Selected Pilot Buses in three VCAs (39-Bus Power System) .....................................26

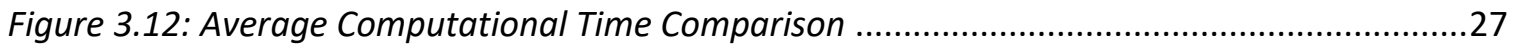

Figure 4.1: Manipulation of a vector with a Linear System ........................................................29

Figure 4.2: All vectors $x_{i}$ around origin ' $O$ ' having same magnitude ' $x$ ' .....................................30

Figure 4.3: Manipulation of input vectors $x_{i}$ with $A$ and corresponding output vectors $y_{i} \ldots . . . . . . . . .30$

Figure 4.4: Decentralized Secondary Voltage Control (SVC) Architecture ....................................34

Figure 4.5: Control Flow diagram for proposed SVC algorithm .....................................................36

Figure 4.6: Reactive power demand under normal conditions (9-Bus Power System)....................37

Figure 4.7: Obtained VCAs and Pilot Buses (9-Bus Power System).............................................38

Figure 4.8: Bus voltages before and after Perturbation in VCA1 (9-Bus Power System)...............40

Figure 4.9: Change in Bus voltages after Perturbation in VCA1 (9-Bus Power System) .................40

Figure 4.10: Bus Voltages before and after Control (9-Bus Power System) ..................................41

Figure 4.11: Change in PV-Bus Voltages after Control (9-Bus Power System) ...............................41

Figure 4.12: Voltage Profile of the System (9-Bus Power System) ..............................................42

Figure 4.13: Bus voltages before and after Perturbation in VCA2 (9-Bus Power System)..............43

Figure 4.14: Change in Bus voltages after Perturbation in VCA2 (9-Bus Power System) ...............43

Figure 4.15: Bus Voltages before and after Control (9-Bus Power System) ..................................44

Figure 4.16: Change in PV-Bus Voltages after Control (9-Bus Power System) ..............................44

Figure 4.17: Voltage Profile of the System (9-Bus Power System) .............................................45

Figure 4.18: Introducing Multiple Perturbations (9-Bus Power System) ......................................46

Figure 4.19: Bus voltages before and after Perturbations (9-Bus Power System)..........................46

Figure 4.20: Change in Bus voltages after Perturbations (9-Bus Power System) ..........................47

Figure 4.21: Bus Voltages before and after Control (9-Bus Power System) ................................47 
Figure 4.22: Change in PV-Bus Voltages after Control (9-Bus Power System) ..............................48

Figure 4.23: Voltage Profile of the System (9-Bus Power System) ................................................48

Figure 4.24: Obtained VCAs and Pilot Buses (39-Bus Power System)..........................................50

Figure 4.25: Bus voltages before and after Perturbation in VCA4 (39-Bus Power System)............52

Figure 4.26: Change in Bus voltages after Perturbation (39-Bus Power System)..........................52

Figure 4.27: Bus Voltages before and after Control (39-Bus Power System) ...............................53

Figure 4.28: Change in PV-Bus Voltages after Control (39-Bus Power System) .............................53

Figure 4.29: Bus voltages before and after Perturbations (39-Bus Power System) ......................54

Figure 4.30: Change in pilot bus voltages after Perturbations (39-Bus Power System) .................55

Figure 4.31: Change in PV bus voltages after control (39-Bus Power System)..............................55

Figure 4.32: Voltage Profile of the System (39-Bus Power System) .............................................56

Figure 4.33: Comparison of Energy Gain between Opt-VC and Sen-VC (39-Bus Power System) ...58 


\section{List of Tables}

Table 3.1: Reactive Power Generation Capacity and Demand (9-Bus Power System) ...................18

Table 3.2: Initial and Optimal Values of $M_{\text {mod }}$ and Avg. Q-Balance (9-Bus Power System) ............20

Table 3.3: Reactive Power Generation Capacity and Demand (39-Bus Power System) .................22

Table 3.4: Initial and Optimal Values of Mmod and Avg. Q-Balance (39-Bus Power System) .......25

Table 4.1: Voltages under Normal Operating Conditions (9-Bus Power System)...........................38

Table 4.2: Data for each Voltage Control Area (9-Bus Power System).........................................39

Table 4.3: Bus Voltages in Different Conditions (9-Bus Power System) ........................................42

Table 4.4: Bus Voltages in Different Conditions (9-Bus Power System) ......................................45

Table 4.5: Bus Voltages in Different Conditions (9-Bus Power System) ........................................48

Table 4.6: Voltages under Normal Operating Conditions (39-Bus Power System)........................49

Table 4.7: Data for each Voltage Control Area (39-Bus Power System).......................................51

Table 4.8: Bus Voltages in Different Conditions (39-Bus Power System) ......................................56 


\section{CHAPTER 1. INTRODUCTION}

\section{Chapter 1}

\section{Introduction}

Voltage and frequency control in power systems have always been considered as two fundamental regulation problems. Frequency regulation through active power control was considered and settled first but voltage control problem has no standard solution yet, Corsi [1]. Voltage regulation through active power control is also theoretically possible but this method is never used in practice except under extreme operating conditions where there are high system security risks, Weedy [2], Taylor [3], Saccomanno [4]. In modern power networks, voltage and frequency control with optimal operation of the network is a big challenge. Voltage regulation in power system is complicated by the fact that it supplies power to a large number of loads and has many generating units including renewable energy resources. As loading conditions are changed, the reactive power requirements of the transmission system vary, Kundur [5]. Moreover, in transmission systems, due to high $\mathrm{X} / \mathrm{R}$ ratio of transmission lines, voltage magnitude is less sensitive to active power and relatively more sensitive to reactive power. Therefore, decoupling voltage control from active power is not only justified but also the common and practical way in power system operations, Sadaat [6]. Considering these facts, fast decoupled load flow assumptions can be used for voltage control.

In transmission systems with $\mathrm{X} \gg \mathrm{R}$, voltages can be controlled by the injection or absorption of reactive power. In general, five methods of injecting reactive power are available: static shunt capacitors, static series capacitors, synchronous compensators, static 


\section{CHAPTER 1. INTRODUCTION}

VAR compensators and STATCOMs, Weedy [2]. Currently, the system voltage profile is kept within normal operating limits by putting a reactive power source at the bus, changing transformer tap ratio or controlling the generator terminal voltage, Zhu [7]. Voltage control based on sensitivity analysis has been a hot research topic for the last few decades. A voltage control technique based on defining voltage control areas using the jacobian matrix is presented in Schlueter [8]. But this method has a high degree of trial and error, Schlueter [9], Aumuller [10]. Lagonotte [11] discusses a method of controlling voltage based on the structure of the network. Voltage control areas are determined based on electrical distance between the buses. This method of finding electrical distance has been applied in Zhong [12] and Nobile [13]. Maharjan [14] identified voltage control areas based on direct relationship between generator's reactive power and load, by finding a sensitivity matrix that relates the reactive power output of a generator to a load.

In this thesis, secondary voltage control for the power system is presented in which a power system is decomposed into voltage control areas initially. A fast-communitydetection algorithm is used to identify these voltage control areas, Iqbal [15]. Then after selecting a pilot point in each area, an optimal voltage control (OVC) algorithm is proposed to obtain new voltage set-points for PV buses by maximizing the effect of change in input (i.e. $\Delta \boldsymbol{V}_{\boldsymbol{P} V}$ ) on change in output (i.e. $\Delta V_{\text {pilot }}$ ) using the FDLF jacobian matrix, so that we can have maximum reactive power reserve and minimum shift of the controls, Iqbal [16-17]. The remainder of the thesis is organized as follows: Literature review is presented in Chapter 2. Optimal network portioning and pilot points selection are explained through case studies in Chapter 3. Chapter 4 presents physical significance of singular value decomposition and its application to optimally control the voltage. Conclusion and future work is discussed in Chapter 5. 


\section{Chapter 2}

\section{Literature Survey}

With the advancements in power system technologies and integration of high level of distributed and environment friendly power sources, the power system constraints become more and more tighter and optimization in narrowing margins has become a critical concern, especially concerning voltage stability and management of static and dynamic reactive power compensation. There are different techniques employed for controlling the network voltage profile, Moursi [18]. Voltage control techniques can be divided into two main categories, Weedy [19]:

1. By injecting reactive power
a. Static shunt capacitors
b. Static series capacitors
c. Synchronous compensators
d. Static VAR compensators (SVC)
e. Static compensators (STATCOM)

2. Using tap changing transformers

Voltage control architecture can be divided into a three-level hierarchical structure, Mousavi [20]:

1. Primary voltage control (PVC)

2. Secondary voltage control (SVC) 


\section{CHAPTER 2. LITERATURE SURVEY}

\section{Tertiary voltage control (TVC)}

Primary voltage control (PVC) is an automatic local voltage control which regulates the voltage of a given bus (e.g. at the stator of the generator) at its set-point. It is referred to the local response of the generator. Automatic voltage regulator (AVR) and static voltage compensator can be used as primary voltage controllers. Secondary voltage control (SVC) is an automatic decentralized control that manages the injection of reactive power within a regional voltage zone by coordinating the actions of local regulators. It is related to the zonal voltage control. Tertiary voltage control (TVC) is the manual optimization of the reactive power flows in a power system and is implemented on the global system level, Rebours [21]. The interactions between these control levels can be managed based on different objectives, time responses, and geographical implications.

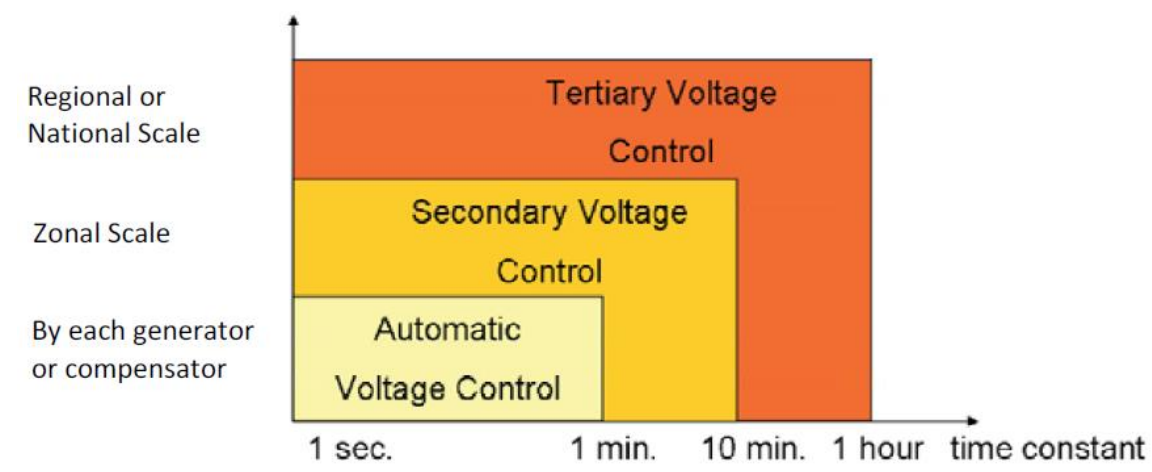

Figure 2.1: Time Space Delineation of a Three Layer Hierarchical Voltage Control Scheme Source: Mousavi [20]

A lot of research has been carried out on voltage control in power systems. Due to the local nature of reactive power and voltage control problems, many approaches exist in literature. Secondary voltage control using pilot buses is one of the most studied problems in power networks. The selection of the pilot points is a critical issue for secondary voltage control, Sancha [22]. SVC scheme involves three steps i.e.

1. Identification of Voltage Control Area (VCAs)

2. Pilot Points Selection

3. Control of Pilot Points Voltages 


\subsection{Identification of Voltage Control Areas (VCAs):}

The first step in applying a secondary voltage control scheme is to partition a large power system into multiple small subsystems called Voltage Control Areas (VCA). Many different network partitioning algorithms are used for the identification of the voltage control areas depending on the objective function under consideration, Daher [23], Gatta [24], Banna [25]. In Lagonotte [26], Shaaban [27] and Zhong [28], a network partitioning algorithm based on electrical distance between nodes is presented by using the Jacobian matrix which describes the bus voltage sensitivities to reactive power injection in the system. The electrical distance, different from geographical distance, is a measure of physical relation among different nodes of a power network. The electrical distance between bus $i$ and bus $j\left(D_{i j}\right)$ is described as

$$
D_{i j}=-\log \left(\alpha_{i j} \cdot \alpha_{j i}\right)
$$

where $a_{i j}$ is a parameter which describes the sensitivity of voltage of bus $i$ to reactive power injection at bus $j$. A general flowchart of network partitioning using electrical distances is shown in Figure 2.2.

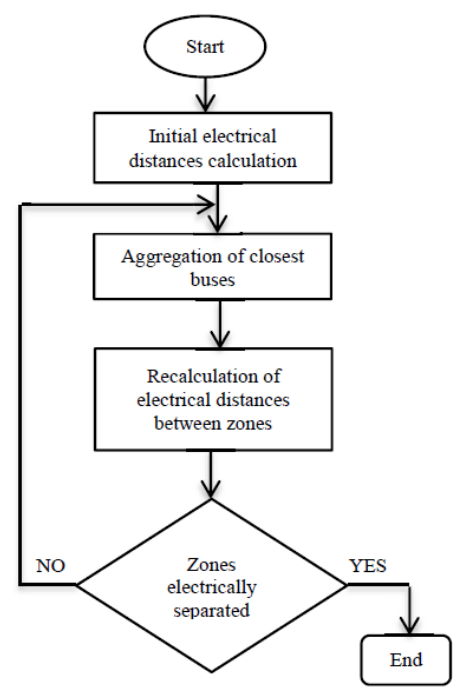

Figure 2.2: Flowchart of Network Partitioning using Electrical Distance Source: Daher [23] 


\section{CHAPTER 2. LITERATURE SURVEY}

An improvement of the electrical-distance based partitioning is the k-means clustering technique which is presented in Satsangi [29], Mehrjerdi [30], Hartigan [31] and Grigoras [32]. This method is based on the Euclidean distance, rather than electrical distance, between the buses which is defined as

$$
F=\sum_{j} \sum_{i}\left\|x_{i}^{(j)}-C_{j}\right\|^{2}
$$

where $x_{i}^{(j)}$ is the data set values of bus $i$ and $C_{j}$ is the centroid of cluster $j$. In this method, $\mathrm{k}$ centroids are arbitrarily (or based on some initial analysis) selected initially. Then buses are distributed among these $\mathrm{k}$ clusters using Euclidean distance between buses and the centroids. After all buses are distributed then new centroids are selected, if these are equal to old centroids then algorithm is ended otherwise it is repeated. A general flowchart of the $\mathrm{k}$-means clustering technique is shown in Figure 2.3.

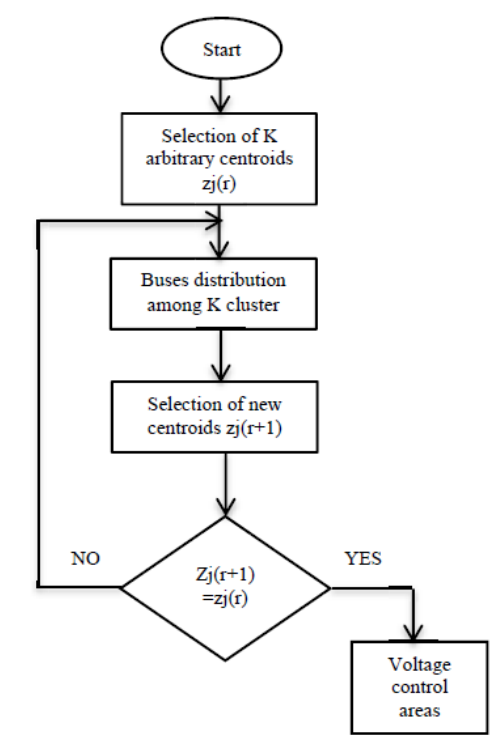

Figure 2.3: Flowchart for K-means Clustering

Source: Daher [23]

It is worth mentioning that in this technique, we need to prespecify the number of cluster we want to divide the network into, which is crucial in some cases. Moreover, it is observed that the partitioning using this technique is dependent on the initial conditions and is 


\section{CHAPTER 2. LITERATURE SURVEY}

influenced by the selection and number of initial centroids, Daher [23]. In Mezquita [33] and Pavão [34], a fuzzy C-means clustering technique was presented as an amelioration of the k-means clustering. In this method, the Euclidean distance is multiplied by a degree of belonging to clusters. Phase angle deviations, calculated by power flow solutions under normal and disturbed conditions, are used as input to fuzzy c-means algorithm.

A myriad of comparative studies can be found in the literature for SVC. In Mori [35], Satsangi [36], Mezquita [37] and Blumsack [38], some of the mostly used network partitioning algorithms for determination of the voltage control areas are studied and compared including electrical-distance-based partitioning, k-means clustering, fuzzy cmeans clustering, genetic-algorithm-based partitioning, Tabu-search-based partitioning, Self-Organizing Map, and Spectral Clustering. In Daher [23], fifteen different network partitioning algorithms are discussed, and an intensive comparative study was performed.

Recently an interesting optimal network partitioning algorithm, called community detection algorithm, was proposed for determination of the voltage control areas in Zhao [39]. This algorithm is based on Girvan-Newman algorithm (original GN algorithm) proposed in Newman [40] and implemented on different social and biological as well as computer generated networks to evaluate the efficacy of the proposed algorithm. Using this algorithm, a network can be decomposed into (optimal number of) smaller networks by detecting dense areas (called communities) using different attributes (e.g. correlation, electrical distance, $\mathrm{V}-\mathrm{Q}$ sensitivity etc.) of a network (i.e. a graph of vertices).

The drawback of this algorithm (original GN-algorithm) is the greedy computations required to find the optimal number of communities (or voltage areas in the context of this research). The computational time required increases exponentially with the size of the system. Later, Girvan and Newman also proposed a fast version of this algorithm (Fast GN-algorithm) which would be used in this research work with some modification to incorporate V-Q sensitivities into it to determine voltage control areas based on voltage and reactive power sensitivities, Iqbal [41]. A detailed introduction to this algorithm would be presented later in this thesis. 


\subsection{Pilot Points Selection}

After decomposing a large power system into smaller subsystems using any of the algorithms described earlier, a load bus (called pilot bus) is selected from each subsystem (i.e. voltage control area) as a representative of the voltage profile of that area. There are many proposed approaches to select these pilot points. A simplest proposed method is to select the buses with highest short circuit capacity as pilot buses. If these bus voltages are kept constant after any disturbance then most of the bus voltages will change very little, Lagonotte [42], Arcidiacono [43]. Keeping this objective, but also including the response of the control generators, another more elaborative approach was suggested in Conejo [44].

Amraee [45] proposed a comprehensive scheme to optimally select pilot points for secondary voltage control. To make the pilot node set robust, they included operational and topological disturbance uncertainties of the power system. A scenario reduction approach was used to reduce the number of combinations of probable states. Genetic algorithm was used to solve the resulted control problem. Finding pilot buses can be a nonlinear and combinatorial problem. By considering different objective functions, e.g. optimizing active power losses and/or voltage deviations and/or reactive power injections, a different sets of pilot buses can be obtained.

A comparison among different pilot point selection techniques has been presented in Gatta [46] and Conejo [47]. They compared the results for voltage area identification and pilot bus selection obtained from four different techniques namely Fuzzy C-Means Clustering Technique, Graph Partitioning, Eigenvalues and Eigenvector of the QV Sensitivity Matrix based technique and K-Means Clustering Technique. They concluded that pilot nodes selection problem is a nontrivial problem and one can reach to distinct but valid solutions depending on the initial premise of objective function.

\subsection{Control of Pilot Points Voltages}

After identification of VCAs and selection of pilot points for each area, the last step in SVC is to maintain the voltages of the pilot buses (which behave as representative of the voltage profiles of each area) at their reference values. Many approaches can be used to 


\section{CHAPTER 2. LITERATURE SURVEY}

bring the pilot bus voltages back to their reference values when the system is disturbed. One simplest approach is to control the pilot bus voltage by controlling the generator bus voltage one by one based on their sensitivity ranking to this pilot bus. Obviously, this objective can be achieved by controlling some of the generators individually or a combination of them depending on the objective function under consideration.

In this thesis, an optimal secondary voltage control technique is proposed with the objective to minimize the shifts of controls (i.e. voltage set-points of generators) using singular value decomposition of voltage-reactive power sensitivity matrix, so that we can have maximum reactive power reserves. An algorithm is proposed to find new set-points for PV-buses to bring pilot bus voltages back to their reference values. Singular values and singular vectors are used to select the voltages of the generators buses (PV buses) so that it would have maximum effect on the pilot bus voltages, Iqbal [48-49]. In this way, we can maximize the change in output (i.e. change in pilot bus voltage) and minimize the shifts of controls (i.e. change in generator bus voltage set-points). 


\section{Chapter 3}

\section{Voltage Control Areas and Pilot Buses}

\subsection{Introduction}

Many networks share a common attribute called community structure. It is the division of a network into subgroups within which the connections are dense, but between which they are sparser. Girvan [55] and Newman [56] proposed a network partitioning approach by detecting naturally dense areas in a network using a measure called modularity. This measure quantifies the quality of a network's partitioning. This approach has been applied to different social, biological and technological networks to discover natural communities, Newman [56], Gupta [57]. In this work, the community detection approach proposed by Newman [56] is being applied in power systems to divide a power network into multiple areas for decentralized voltage control. The advantage of this approach is that it not only detects the naturally dense areas but also gives optimal number of communities a system should be divided into.

There are many characteristics which can be used for discovering natural divisions in power systems e.g. correlation coefficient, path lengths, $V$ - $Q$ sensitivity and electrical distances between vertices, Newman [58-59]. In this paper, I used network adjacency matrix based on physical connections of the network and incorporated $V$ - $Q$ sensitivity in it to minimize inter-community voltage-reactive power sensitivity. A modified modularity 


\section{CHAPTER 3. VOLTAGE CONTROL AREAS AND PILOT BUSES}

measure was used to consider community structures in physical power networks, $V-Q$ sensitivity and reactive power balance. This measure was proposed by Zhao [60] but his algorithm is very greedy in terms of computations required because he was checking all possible combinations of nodes (to merge in each iteration) since $V-Q$ sensitivity matrix is not a sparse matrix. I improved this algorithm by considering the physical topology of a power system network because only those nodes must be combined in a community (i.e. voltage control area) which are physically connected (i.e. have a physical power line between themselves). The algorithm proposed is based on the fast-community-detection approach proposed by Newman [61].

In this chapter, an optimal network partitioning algorithm is presented to identify voltage control areas by discovering the natural community structures in the power network, minimizing the voltage - reactive power sensitivity between communities and maximizing the balance in reactive power generation capacity and demand to make sure that each community is self-sufficient in satisfying its reactive power demand to be able to keep voltage profile within nominal range. After decomposing the system into subsystems, pilot points are selected in each subsystem to represent the voltage profile of the whole subsystem.

The presented optimal network partitioning approach is formulated in Section 3.2. Section 3.2.3 describes all steps involved in the presented algorithm through a control flow diagram. Section 3.3 describes pilot point selection technique used in this research. Simulation results through case studies and performance comparison are presented in Section 3.4.

\subsection{Optimal Network Partitioning}

\subsubsection{Modularity (a quantification of the quality of a partition)}

Newman [58] studied that real-world networks show some kind of mixing patterns. He considered assortative mixing (the tendency for vertices in networks to be connected to other vertices that are like (or unlike) them in some way) in networks according to discrete node characteristics. Such mixing can be characterized by a quantity $e_{i j}$, which is defined as the fraction of edges in a network that connect a node of type $i$ to one of type $j$. For 


\section{CHAPTER 3. VOLTAGE CONTROL AREAS AND PILOT BUSES}

undirected networks (or graphs), it is symmetric i.e. $e_{i j}=e_{j i}$. Let's define a mixing matrix $\mathbf{E}$ which contains all such quantities (i.e. $\mathbf{E}=\left[e_{i j}\right]$ ). It satisfies the properties described in (3.1).

$$
\sum_{i j} e_{i j}=1, \sum_{j} e_{i j}=a_{i}, \sum_{i} e_{i j}=b_{j}
$$

where $a_{i}$ is the sum of $i^{\text {th }}$ row and $b_{j}$ is the sum of $j^{\text {th }}$ column of mixing matrix $\boldsymbol{E}$. Let's define a column vector $\boldsymbol{a}$ as row-sum-vector (containing sum of each row) and $\boldsymbol{b}$ as columnsum-vector (containing sum of each column). The quality function (modularity) proposed by Girvan and Newman [56] can be used to quantify the quality of a particular partition of a network. It measures fraction of the edges in the network that connect vertices of the same type (i.e., intra-community edges) minus the expected value of the same quantity in a network with the same community divisions but random connections between the vertices. The higher the difference between these quantities (i.e. fraction of intracommunity edges and its expected value), stronger will be the community structure in the network. For an undirected network, it can be described as in (3.2), which lies in general in the range $-1 \leq M \leq 1$.

$$
M=\operatorname{Tr}(\boldsymbol{E})-\boldsymbol{a}^{T} \boldsymbol{b}^{T}
$$

\subsubsection{Modified Modularity}

To divide the network for voltage control using reactive power injection, we need to incorporate reactive power - voltage sensitivity into modularity. For this purpose, we used electrical distance which describes $V$ - $Q$ sensitivity relation between two buses. Lower the electrical distance between two buses, higher is the $V$ - $Q$ coupling between them. To calculate the electrical distance matrix $\left(\mathbf{D}_{\mathbf{e}}\right)$ of the power network, $V-Q$ sensitivity matrix is used. Using fast decoupled load flow assumptions, the change in bus voltage magnitudes can be approximated by (3.3).

$$
\Delta V=-[B "]^{-1} \Delta Q=S^{V Q} \Delta Q
$$

where $\boldsymbol{S}^{\boldsymbol{V} \boldsymbol{Q}}$ is the sensitivity matrix describing the change in bus voltages w.r.t change in reactive power injection (near the operating point) in the system and $\boldsymbol{B}^{\prime \prime}$ is obtained from 


\section{CHAPTER 3. VOLTAGE CONTROL AREAS AND PILOT BUSES}

the susceptance matrix (B) and is defined as in (3.3a), see Appendix for details. For an $n$ bus power system, $\boldsymbol{\Delta} \boldsymbol{V}$ and $\boldsymbol{\Delta Q}$ are column vectors of order $n x l$ each and $\boldsymbol{S}^{\boldsymbol{V} \boldsymbol{Q}}$ is an $n x n$ matrix.

$$
\begin{aligned}
& B_{k i}^{\prime \prime}=-B_{k i} \quad \forall k, k \neq i \\
& B_{k k}^{\prime \prime}=-B_{k k}-\sum_{i=1}^{n} B_{k i}
\end{aligned}
$$

\subsubsection{Electrical Distance:}

The $V-Q$ sensitivity matrix ( $\boldsymbol{S}^{V \boldsymbol{Q}}$ ) can be used to quantify the voltage proximities of the buses. The magnitude of the coupling in terms of voltage between two nodes of an electrical system can be reflected and quantified by the maximum attenuation of voltage variations between these two nodes. These attenuations are easy to obtain from the $V-Q$ sensitivity matrix $\boldsymbol{S}^{V \boldsymbol{Q}}\left(=\left[\frac{\partial V}{\partial Q}\right]\right)$. To do so, one just needs to divide the elements of each column by the corresponding diagonal term, Lagonotte [63]. A matrix of attenuations between all the nodes of system, whose terms are written as $\left[\alpha_{i j}\right]$, is then calculated as in (3.5).

$$
\Delta V_{i}=\alpha_{i j} \Delta V_{j}
$$

where

$$
\alpha_{i j}=\frac{\left[\frac{\partial V_{i}}{\partial Q_{j}}\right]}{\left[\frac{\partial V_{j}}{\partial Q_{j}}\right]}=\frac{S_{i j}^{V Q}}{S_{j j}^{V Q}}
$$

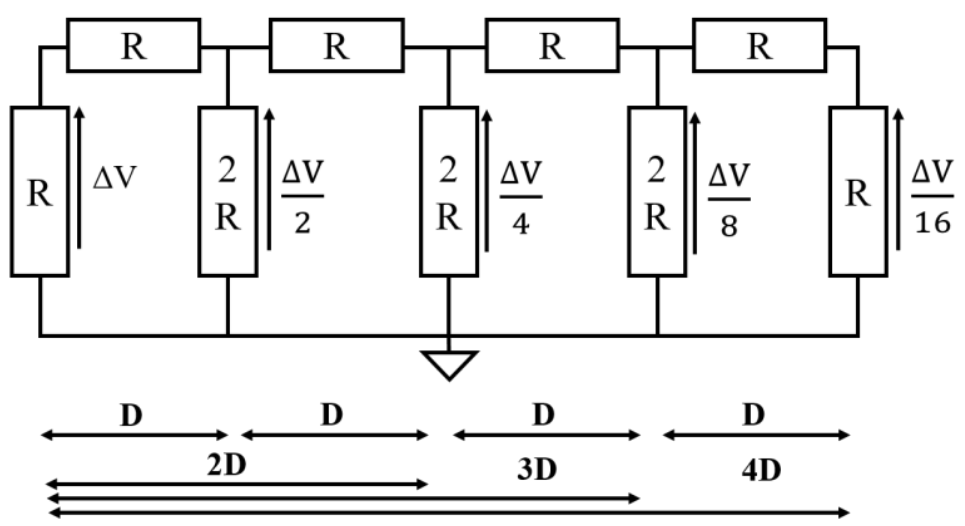

Fig. 3.1. The attenuations on a simple example

For easy manipulation of data and efficient application of different of algorithms, a mathematical structure of distances between all the nodes is defined by a distance matrix 


\section{CHAPTER 3. VOLTAGE CONTROL AREAS AND PILOT BUSES}

(De). Attenuation in voltage variations is shown in Fig. 3.1 for a simple system. It is necessary to affect the product of attenuations to change over from a couple of nodes to another. To switch over from a product to a sum, it is possible to take the logarithm of attenuation as definition of the distance between two nodes as given by (3.6).

$$
D_{e}^{i j}=\left|\log \alpha_{i j}\right|
$$

However, to obtain symmetrical distances, the formulation in (3.7) is taken as definition of the electrical distance between two nodes $i$ and $j$ :

$$
D_{e}^{i j}=D_{e}^{j i}=\left|\log \left(\alpha_{i j} \cdot \alpha_{j i}\right)\right|
$$

Smaller the electrical distance $\left(D_{e}^{i j}\right)$ between two nodes $i$ and $j$, higher is the $V-Q$ coupling between them indicating strong $V-Q$ sensitivity. Now we use this electrical distance matrix $\left(\mathbf{D}_{\mathbf{e}}\right)$ as the edge matrix $\left(\boldsymbol{E}^{V Q}\right)$ of the virtual $V-Q$ sensitivity graph of the power network whose edges would indicate the electrical distance (and hence $V$ - $Q$ sensitivity) between the buses in the power system. So $\boldsymbol{E}^{\boldsymbol{V} \boldsymbol{Q}}$ is also a symmetric matrix like $\boldsymbol{D}_{\boldsymbol{e}}$. To make it compatible with modularity $(M)$, it is normalized to satisfy the first property as described in $(3.1)$.

$$
\boldsymbol{E}^{\boldsymbol{V Q}}=\left[e_{i j}^{V Q}\right]=\boldsymbol{D}_{\boldsymbol{e}}=\left[D_{e}^{i j}\right]
$$

We define the average $V$ - $Q$ sensitivity $\left(\gamma_{C k}\right)$ of $k^{\text {th }}$ community $\left(C_{k}\right)$ as in (3.9) by taking the average of weighted edges $\left(e_{i j}^{V Q}\right)$ over all edges in a community.

$$
\gamma_{C k}=\underset{\forall i, j \in C_{k}}{\operatorname{avg}}\left(e_{i j}^{V Q}\right)
$$

A smaller value of $\gamma_{C k}$ means that voltage profile in community $k$ is more sensitive to reactive power injection within that community (indicating smaller average electrical distance and hence higher $V$ - $Q$ sensitivity between buses in that communities). The reactive power imbalance $\left(\lambda_{C k}\right)$ of $k^{\text {th }}$ community is described as in (3.10).

$$
\lambda_{C k}=\left\{\begin{aligned}
0, & Q_{C k}^{S}>Q_{C k}^{D} \text { or } Q_{C k}^{D}=0 \\
1-\left|\frac{Q_{C k}^{S}}{Q_{C k}^{D}}\right|, & \text { otherwise }
\end{aligned}\right.
$$




\section{CHAPTER 3. VOLTAGE CONTROL AREAS AND PILOT BUSES}

where $Q_{C k}^{S}$ is the maximum reactive power a community $k$ can supply (maximum $Q$ generation capacity of the community) and $Q_{C k}^{D}$ is the total reactive power demand in $k^{\text {th }}$ community. A smaller value of $\lambda_{C k}$ means that $k^{\text {th }}$ community can satisfy most of its reactive power demand (i.e. minimum reactive power imbalance).

To incorporate $V-Q$ sensitivity into modularity $(M)$ and make each community selfsufficient in satisfying its reactive power demand (so that each community can maintain its voltage profile within nominal limits), a modified modularity $\left(M_{m o d}\right)$ is used as described in $(3.11)$.

$$
M_{\text {mod }}=M-\underset{\forall k}{\operatorname{avg}}\left(\gamma_{C k}+\lambda_{C k}\right)
$$

A high value of $M_{m o d}$ indicates that intra-community edges are dense compared to intercommunity edges while each community can fulfill most of its $Q$-demand and its voltage profile is less sensitive to $Q$-injection in other communities due to high electrical distances among communities (i.e. inter-community electrical distance).

\subsubsection{Optimal Network Partitioning Algorithm}

Let $\mathcal{E}$ (edge-set) be the set of all inter-community edges. The presented optimal network partitioning algorithm can be described by following steps:

1 Consider each node (i.e. a bus in power systems) as a separate community and compute initial modified modularity $\left(M_{\bmod }^{0}\right)$.

2 Select an edge (e) i.e. a branch in power systems, from edge-set $(\varepsilon)$, consider the communities at its ends as one community and compute new modified modularity $\left(M_{\text {mod }}^{n e w}\right)$ using (3.2) - (3.11). Then calculate $\Delta M_{\text {mod }}^{e}=M_{\text {mod }}^{n e w}-$ $M_{\text {mod }}^{0}$.

3 Repeat step-2 for all edges in the edge-set $(\mathcal{E})$ taking only one edge at a time and build a vector $\left(\boldsymbol{\Delta} \boldsymbol{M}_{\boldsymbol{m o d}}\right)$ as described in (3.12).

$$
\Delta \boldsymbol{M}_{\text {mod }}=\left[\Delta M_{\text {mod }}^{e}\right], \quad \forall e \in \mathcal{E}
$$

4 Identify the edge $\left(e_{m}\right)$ which is giving maximum change in modified modularity $\overline{\Delta M}_{\text {mod }}=\max \left(\Delta \boldsymbol{M}_{\text {mod }}\right)$. 
5 IF: $\overline{\Delta M}_{\text {mod }}>0$, merge the two communities at the ends of $e_{m}$ in one community and update initial modified modularity $\left(M_{m o d}^{0}\right)$ as in (3.13). Exclude $e_{m}$ from the edge-set $(\mathcal{E})$ and return to step 2.

$$
M_{\text {mod }}^{0}=M_{\text {mod }}^{0}+\overline{\Delta M}_{\text {mod }}
$$

ELSE: Terminate the algorithm. The number of communities at this stage are optimal number of communities (because $M_{\text {mod }}$ is already maximized and can't be improved further). Identify the buses in communities and return $M_{\text {mod }}^{o p t}=$ $M_{\text {mod }}^{0}$.

The control flow diagram of the presented optimal network partitioning algorithm is shown in Fig. 3.2.

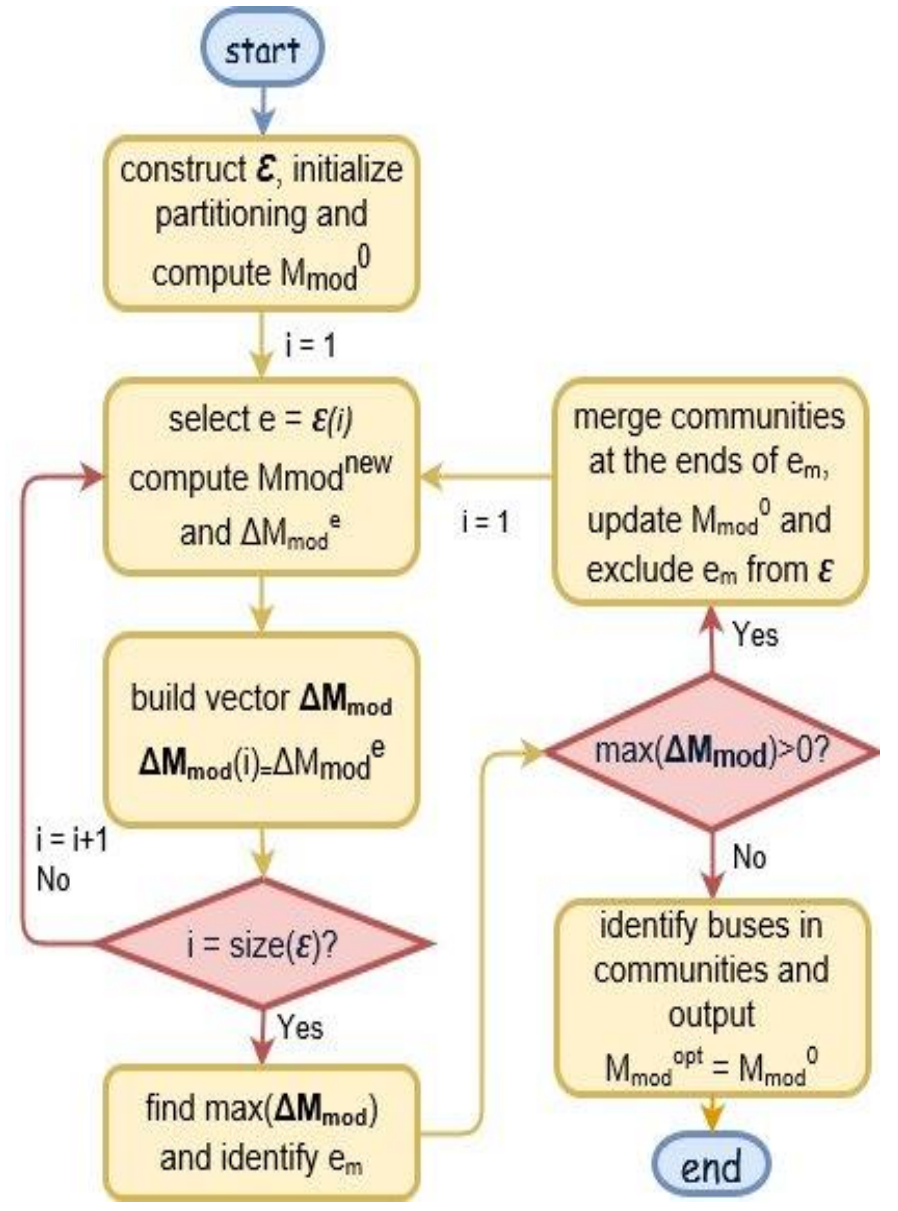

Fig. 3.2. Optimal Network Partitioning Algorithm Flowchart

\subsection{Pilot Points Selection}

The selection of the pilot points is one of most important part in making secondary voltage control effective. A pilot point/bus is a load bus which represents the voltage profile 
of the whole voltage control area. If these pilot point voltages are kept constant before and after disturbance(s), it is considered that all other bus voltages in that area would vary very little. Since a pilot node is a representative of the whole voltage control area, it is selected with great care and consideration. There are many approaches which have been developed over last few decades. The simplest approach is to select the node with highest short circuit capacity in the area as pilot node. In this section, we would use electrical distances to identify pilot points for each control area.

\subsubsection{Pilot Bus Selection using Electrical Distances}

Since electrical distances represent the physical relationship between voltage variations of the buses, it can be used to select the pilot bus in a control area. A bus having minimum electrical distance in a control area would be most sensitive to the reactive power disturbances on the buses in that area and hence can be chosen to represent the voltage profile of the whole area. If its voltage is kept constant before and after disturbance(s) using secondary voltage control, then the voltage variations on remaining buses would be small.

After calculating the electrical distances of all the buses using (3.7), a bus $i$ would be selected as a pilot bus in a community (or VCA), $\mathrm{C}_{\mathrm{k}}$, if it satisfies the condition in (3.14).

$$
\min _{i}\left(\sum_{\boldsymbol{j} \in \boldsymbol{C}_{\boldsymbol{k}}} \boldsymbol{D}_{\boldsymbol{e}}^{\boldsymbol{i j}} ; \forall \boldsymbol{i} \in \boldsymbol{C}_{\boldsymbol{k}}\right)
$$

This approach for pilot points selection using electrical distances gives good observability, Nivine [64].

\subsection{Case Studies and Simulation Results}

The presented optimal network partitioning algorithm and pilot point selection approach were tested on standard IEEE test power systems, namely 9-bus and 39-bus systems using a dual core $2.13 \mathrm{GHz}$ machine having 6GB of random access memory (RAM). All simulations results were obtained using MATLAB-R2017a and MATPOWER 6.0 [65-66]. 


\subsubsection{9-Bus Power Network}

This system has reactive power generation on three buses (bus 1-3). The reactive power generation capacity and demand on each bus is given in Table 3.1.

TABLE 3.1. Reactive Power Generation Capacity and Demand (9-Bus Power

\begin{tabular}{|c|c|c|}
\hline \multicolumn{3}{|c|}{ System) } \\
\hline Bus \# & $\begin{array}{c}Q^{S} \\
(M V A R)\end{array}$ & $\begin{array}{c}Q^{\mathrm{D}} \\
\text { (MVAR) }\end{array}$ \\
\hline 1 & 100 & 0 \\
\hline 2 & 100 & 20 \\
\hline 3 & 100 & 30 \\
\hline 4 & 0 & 20 \\
\hline 5 & 0 & 10 \\
\hline 6 & 0 & 20 \\
\hline 7 & 0 & 10 \\
\hline 8 & 0 & 20 \\
\hline 9 & 0 & 30 \\
\hline Total & 300 & 160 \\
\hline
\end{tabular}

The electrical distances matrix $\left(\boldsymbol{D}_{\boldsymbol{e}}=\left[D_{e}^{i j}\right]\right)$ for 9-bus power system under consideration can be calculated using (3.3) - (3.7). The matrix is shown in Fig. 3.3.

$\left[\begin{array}{lllllllll}0.000 & 0.157 & 0.187 & 0.013 & 0.045 & 0.173 & 0.281 & 0.143 & 0.041 \\ 0.157 & 0.000 & 0.101 & 0.144 & 0.284 & 0.087 & 0.038 & 0.014 & 0.082 \\ 0.187 & 0.101 & 0.000 & 0.174 & 0.089 & 0.013 & 0.050 & 0.087 & 1.000 \\ 0.013 & 0.144 & 0.174 & 0.000 & 0.032 & 0.161 & 0.268 & 0.131 & 0.028 \\ 0.045 & 0.284 & 0.089 & 0.032 & 0.000 & 0.076 & 0.174 & 0.270 & 0.073 \\ 0.173 & 0.087 & 0.013 & 0.161 & 0.076 & 0.000 & 0.037 & 0.074 & 0.987 \\ 0.281 & 0.038 & 0.050 & 0.268 & 0.174 & 0.037 & 0.000 & 0.024 & 0.123 \\ 0.143 & 0.014 & 0.087 & 0.131 & 0.270 & 0.074 & 0.024 & 0.000 & 0.068 \\ 0.041 & 0.082 & 1.000 & 0.028 & 0.073 & 0.987 & 0.123 & 0.068 & 0.000\end{array}\right]$

Fig. 3.3. Electrical Distance Matrix [D $\left.D_{i j}\right]$ (9-Bus Power System) 


\section{CHAPTER 3. VOLTAGE CONTROL AREAS AND PILOT BUSES}

\subsubsection{Voltage Control Areas/ Communities}

After applying the optimal network partitioning algorithm, the system was divided into three voltage control areas (communities) each containing three buses (because it is a highly random network). Fig. 3.4 shows single line diagram of the system along with the buses in each community after partitioning.

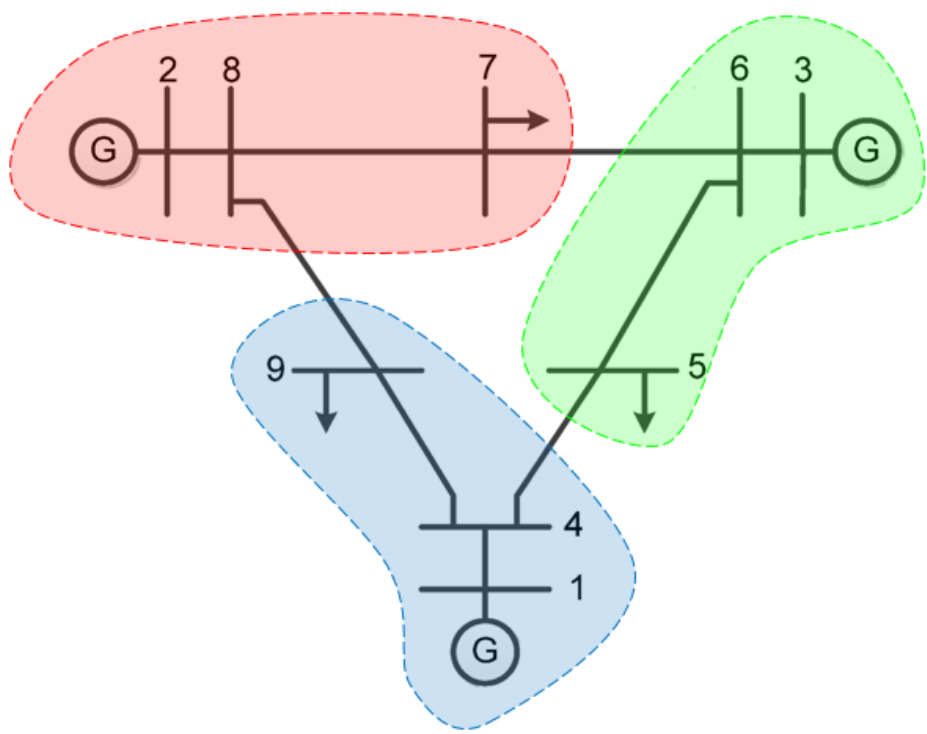

Fig. 3.4. Buses in each Community after Optimal Partitioning (9-Bus Power System)

The system dendrogram showing buses merging sequence and changes in modified modularity are shown in Fig. 3.5. 

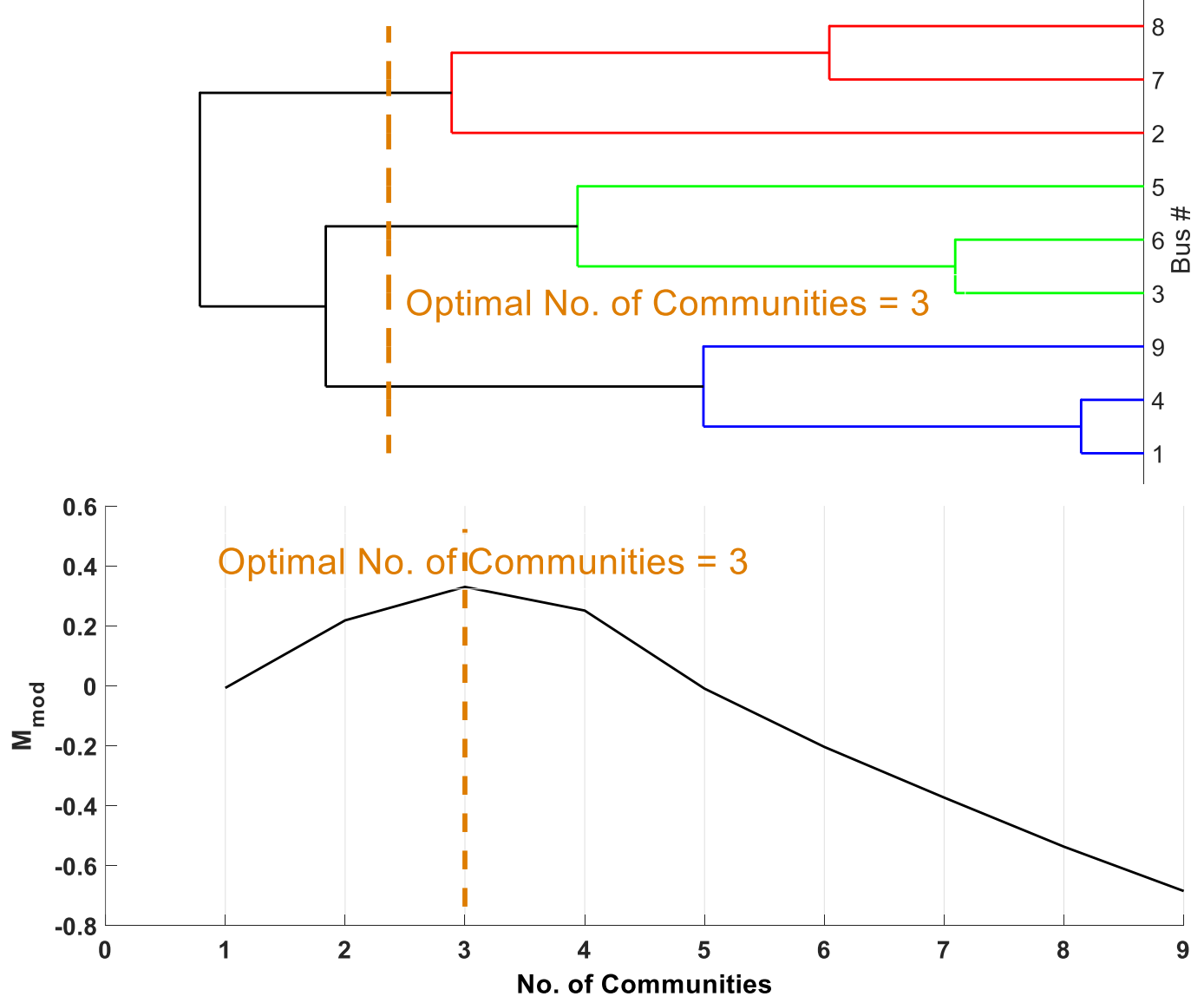

Fig. 3.5. Dendrogram and $M_{\text {mod }}$ variations (9-Bus Power System)

Initial and optimal values of $M_{\text {mod }}$ are given in Table 3.2. Average reactive power balance over all communities is $100 \%$, showing that each community can independently satisfy its reactive power demand and hence can maintain its voltage profile within normal operating limits.

TABLE 3.2. Initial and Optimal Values of $M_{\text {mod }}$ and Avg. Q-Balance (9-Bus Power System)

\begin{tabular}{|c|c|}
\hline Optimal No. of Communities & 3 \\
\hline Initial $\mathbf{M}_{\mathrm{mod}}$ & -0.6852 \\
\hline Optimal $\mathbf{M}_{\mathrm{mod}}$ & 0.3307 \\
\hline Average Q-Balance & $100 \%$ \\
\hline
\end{tabular}




\section{CHAPTER 3. VOLTAGE CONTROL AREAS AND PILOT BUSES}

\subsubsection{Pilot Points Identification}

To identify the pilot points using electrical distances $\left(\left[\mathrm{D}^{\mathrm{ij}}\right]\right)$, we use condition (3.14). This condition selects the load bus as a pilot point in a VCA which has minimum electrical distance from all other buses in that VCA. In other words, it would select that bus as the pilot bus which is located at the electrical center of the VCA. The pilot buses for the three VCAs discovered n previous section are shown in Fig. 3.6.

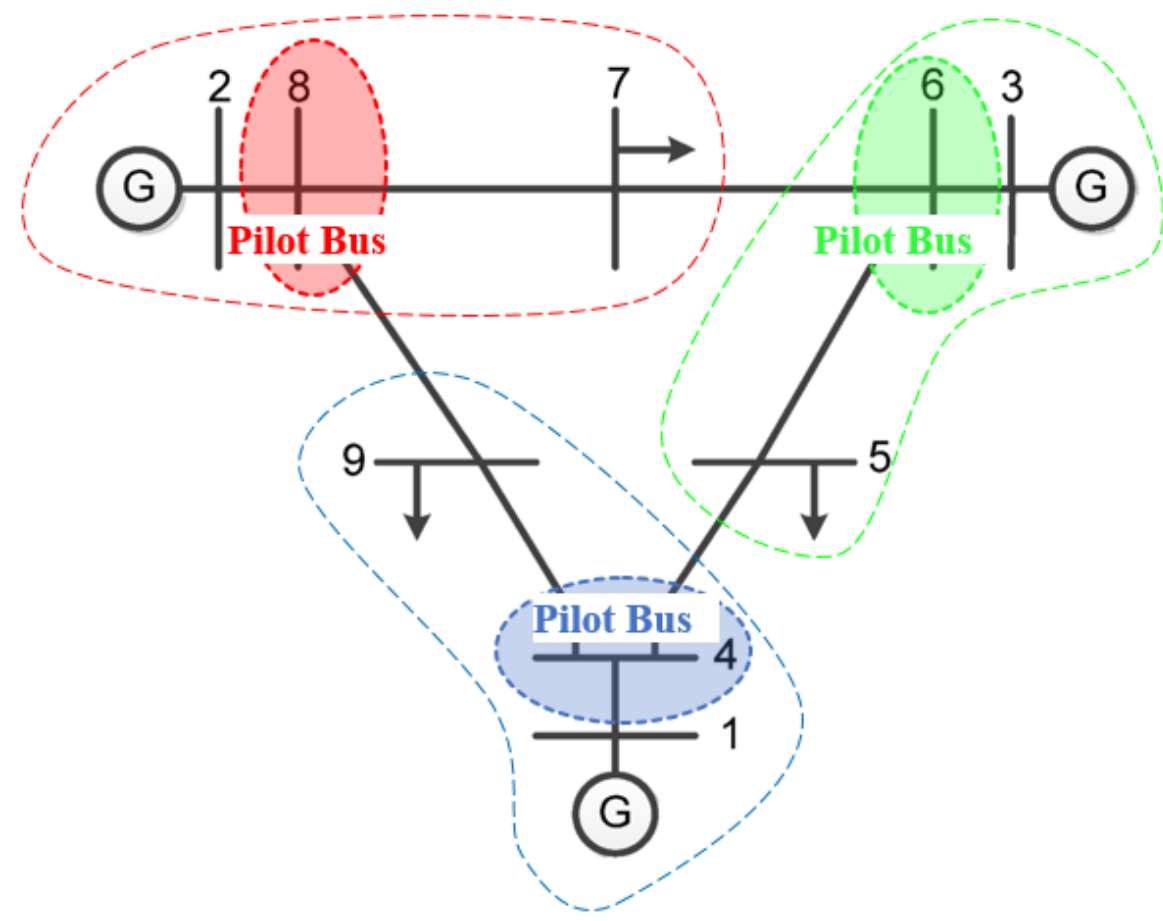

Fig. 3.6. Selected Pilot Buses in three VCAs (9-Bus Power System)

\subsubsection{9-Bus Power Network}

This system has reactive power generation on ten buses (bus 30-39). The single line diagram of the system is shown in Fig. 3.7. The reactive power generation capacity and demand on each bus is given in Table 3.3 . 


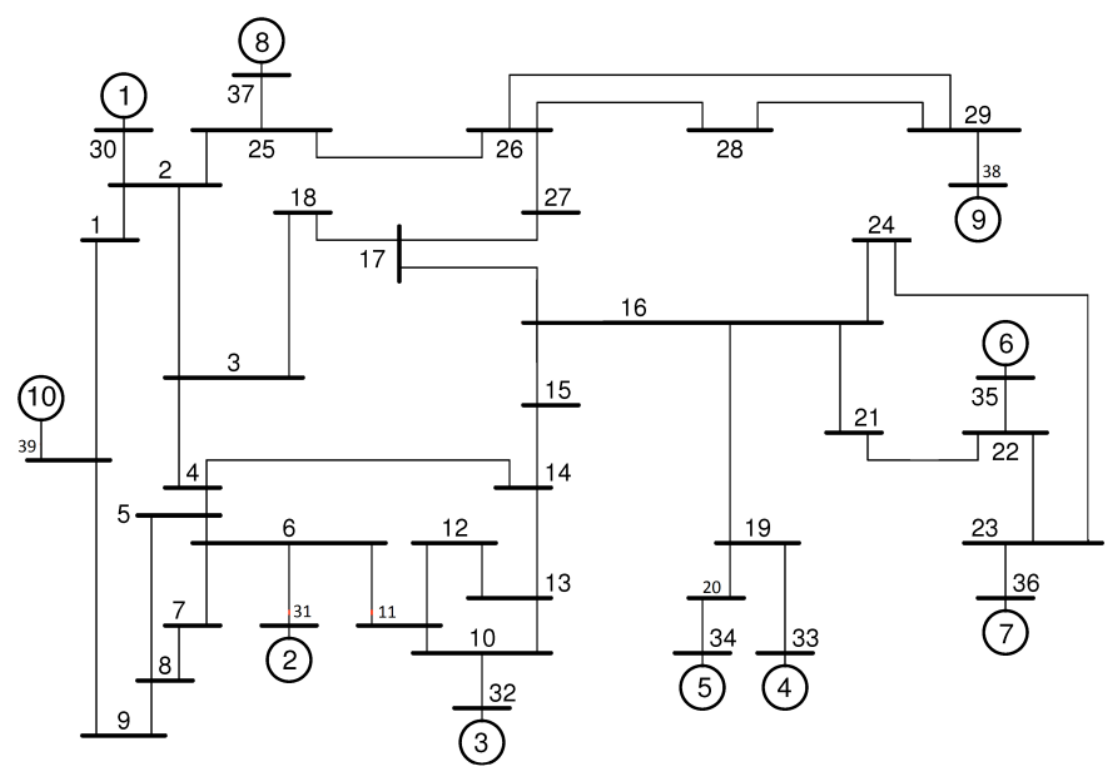

Fig. 3.7. Single Line Diagram (39-Bus Power System)

TABLE 3.3. Reactive Power Generation Capacity and Demand on each Bus (39-Bus Power System)

\begin{tabular}{|c|c|c|c|c|c|c|c|c|}
\hline Bus & $\begin{array}{l}\text { Q }_{\text {support }} \\
\text { (MVAR) }\end{array}$ & $\begin{array}{l}Q_{\text {demand }} \\
\text { (MVAR) }\end{array}$ & Bus & $\begin{array}{l}\text { Q }_{\text {support }} \\
\text { (MVAR) }\end{array}$ & $\begin{array}{l}Q_{\text {demand }} \\
\text { (MVAR) }\end{array}$ & Bus & $\begin{array}{l}\text { Q }_{\text {support }} \\
\text { (MVAR) }\end{array}$ & $\begin{array}{l}Q_{\text {demand }} \\
\text { (MVAR) }\end{array}$ \\
\hline 1 & 0 & 44.2 & 14 & 0 & 0 & 27 & 0 & 75.5 \\
\hline 2 & 0 & 0 & 15 & 0 & 153 & 28 & 0 & 27.6 \\
\hline 3 & 0 & 2.4 & 16 & 0 & 32.3 & 29 & 0 & 26.9 \\
\hline 4 & 0 & 184 & 17 & 0 & 0 & 30 & 400 & 0 \\
\hline 5 & 0 & 0 & 18 & 0 & 30 & 31 & 300 & 4.6 \\
\hline 6 & 0 & 0 & 19 & 0 & 0 & 32 & 300 & 0 \\
\hline 7 & 0 & 84 & 20 & 0 & 103 & 33 & 250 & 0 \\
\hline 8 & 0 & 176.6 & 21 & 0 & 115 & 34 & 167 & 0 \\
\hline 9 & 0 & -66.6 & 22 & 0 & 0 & 35 & 300 & 0 \\
\hline 10 & 0 & 0 & 23 & 0 & 84.6 & 36 & 240 & 0 \\
\hline 11 & 0 & 0 & 24 & 0 & -92.2 & 37 & 250 & 0 \\
\hline 12 & 0 & 88 & 25 & 0 & 47.2 & 38 & 300 & 0 \\
\hline 13 & 0 & 0 & 26 & 0 & 17 & 39 & 300 & 250 \\
\hline \multicolumn{7}{|c|}{ Total } & 2807 & 1387.1 \\
\hline
\end{tabular}

The electrical distances matrix $\left(\boldsymbol{D}_{\boldsymbol{e}}=\left[D_{e}^{i j}\right]\right)$ for 39-bus power system is shown in Fig. 3.8. 


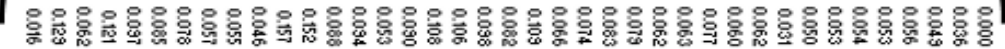

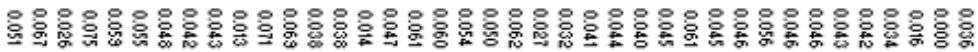

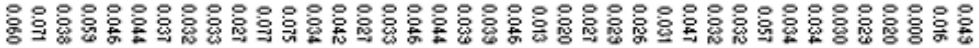

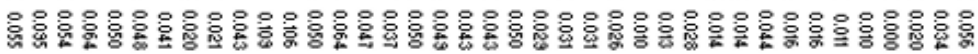

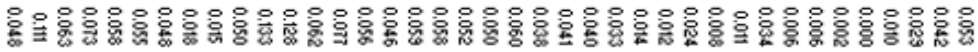

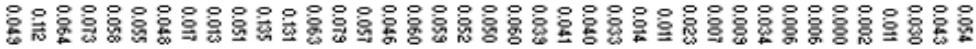

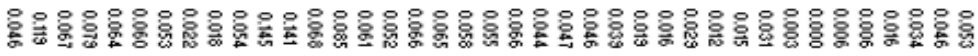

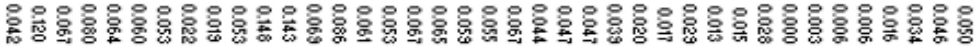

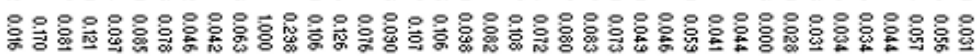

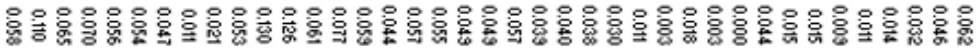

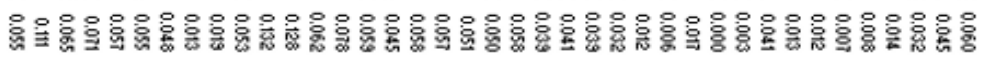

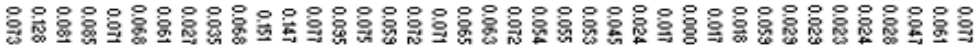

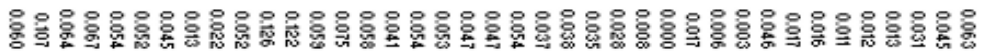

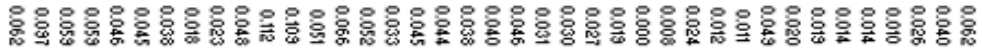

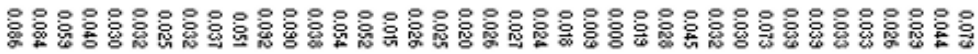

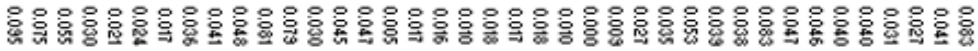

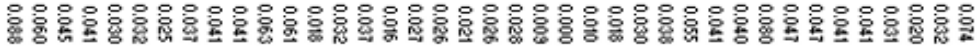

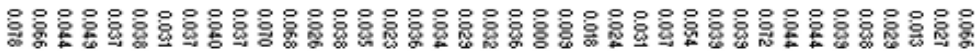

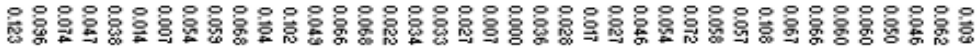

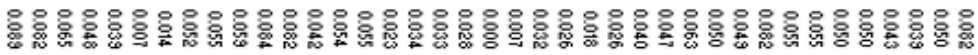

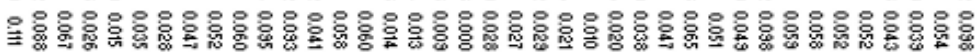

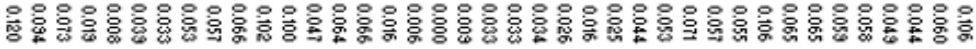

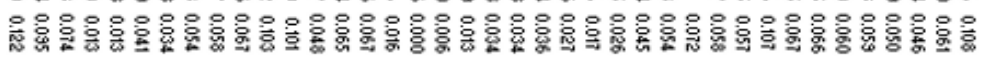

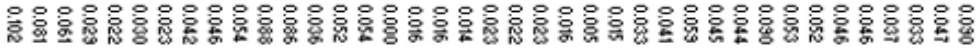

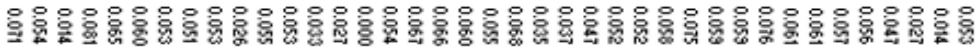

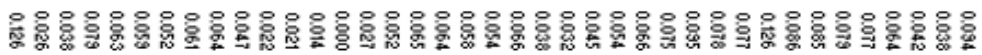

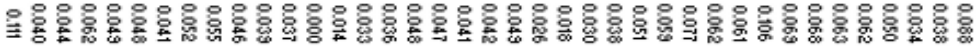

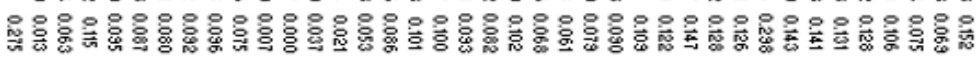

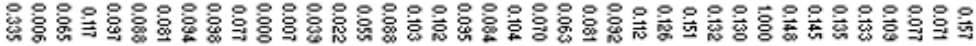

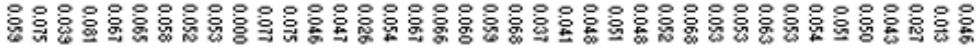

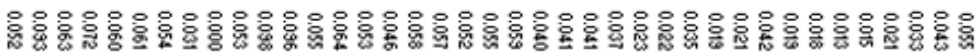

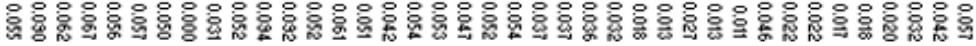

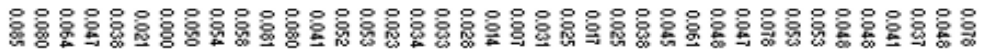

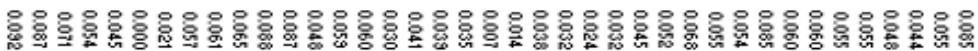

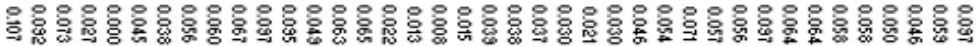

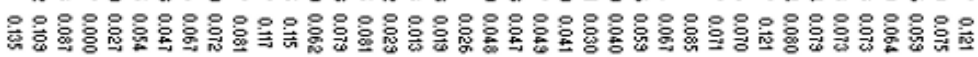

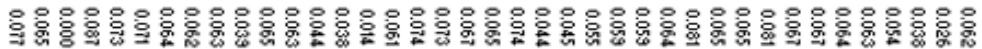

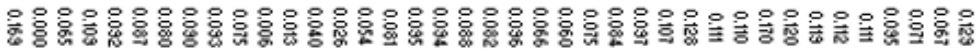

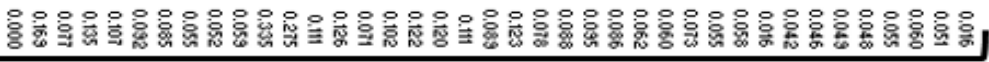

Fig. 3.8. Electrical Distance Matrix [ $D_{i j}$ ] (39-Bus Power Network) 


\section{CHAPTER 3. VOLTAGE CONTROL AREAS AND PILOT BUSES}

\subsubsection{Voltage Control Areas/ Communities}

After applying the optimal network partitioning algorithm, the system was divide into six communities. The system dendrogram showing buses merging sequence and changes in modified modularity are shown in Fig. 3.9.
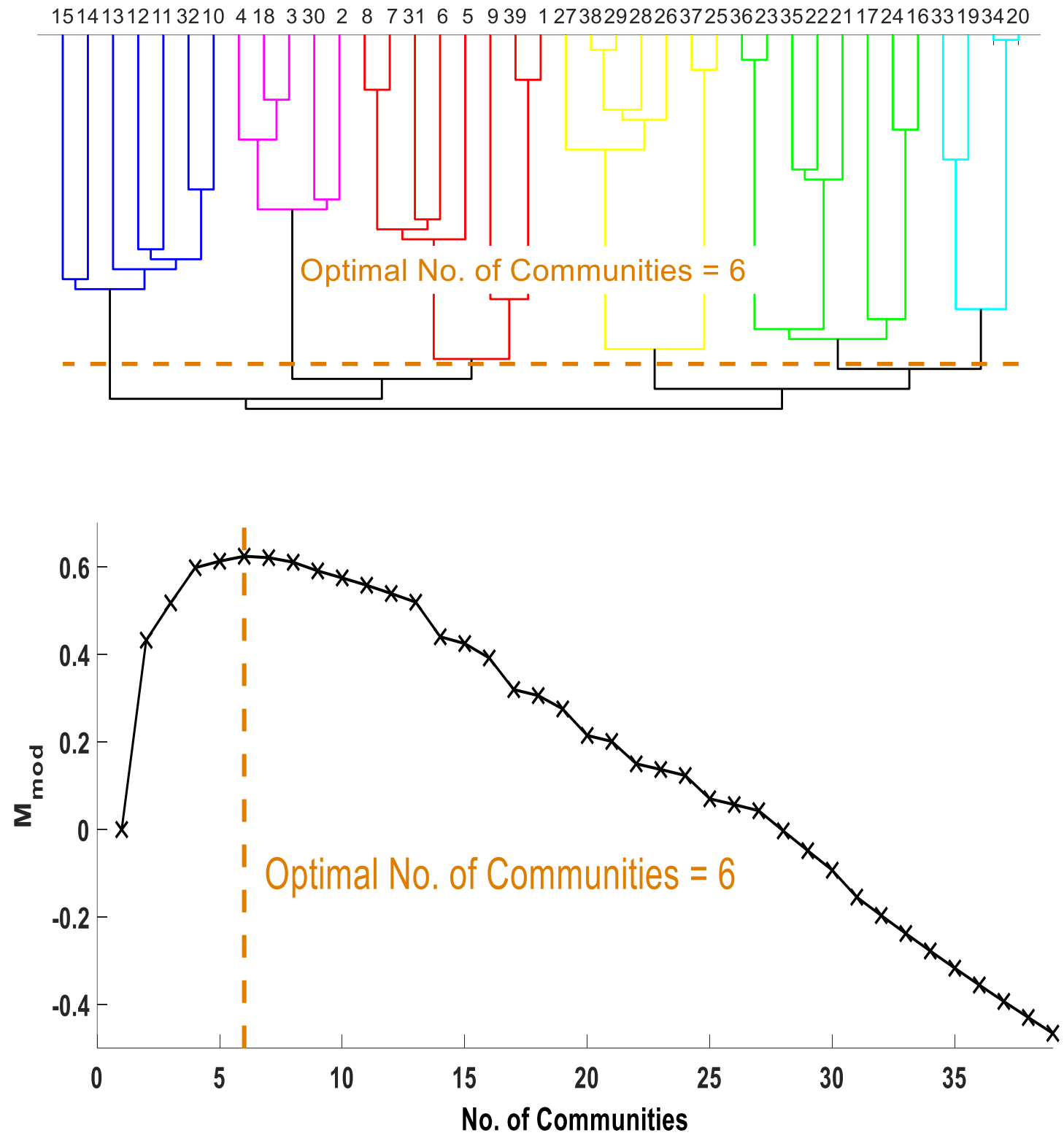

Fig. 3.9. Dendrogram and $M_{\text {mod }}$ variations (39-Bus Power System) 


\section{CHAPTER 3. VOLTAGE CONTROL AREAS AND PILOT BUSES}

Fig. 3.10 shows single line diagram of the system along with the buses in each community after partitioning. Initial and optimal values for $\mathbf{M}_{\bmod }$ are given in Table 3.4. Average reactive power balance in all communities is $100 \%$.

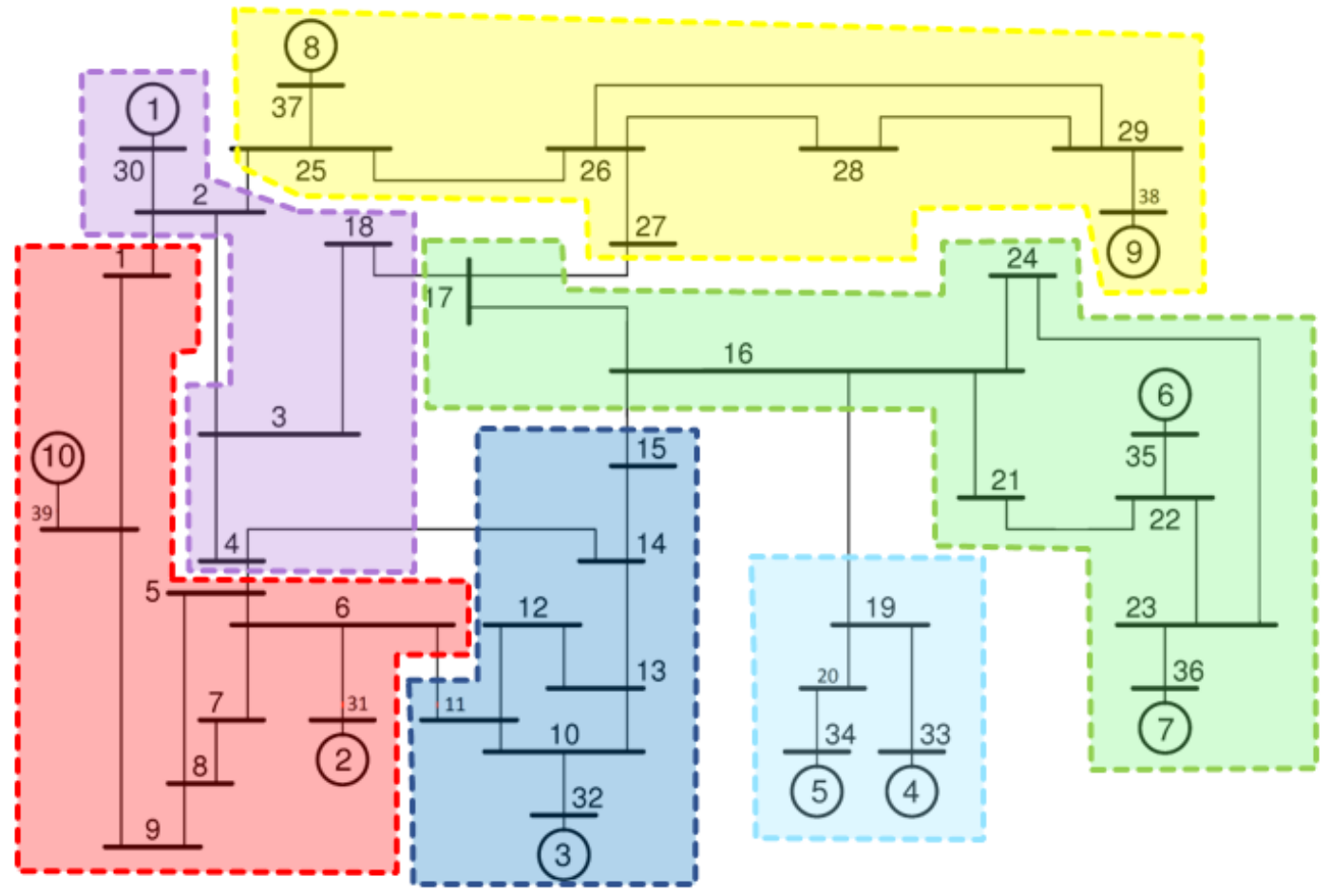

Fig. 3.10. Buses in each Community (39-Bus Power System)

TABLE 3.4. Initial and Optimal Values of $M_{\text {mod }}$ and Avg. Q-Balance (39-Bus Power System)

\begin{tabular}{|c|c|}
\hline Optimal No. of Communities & 6 \\
\hline Initial Mmod & -0.4661 \\
\hline Optimal Mmod & 0.6241 \\
\hline Average Q-Balance & $100 \%$ \\
\hline
\end{tabular}




\subsubsection{Pilot Points Identification}

The pilot points using electrical distances (i.e. electrical centers) of six voltage control areas shown in Fig. 3.10 for 39-bus power system are shown in Fig. 3.11.

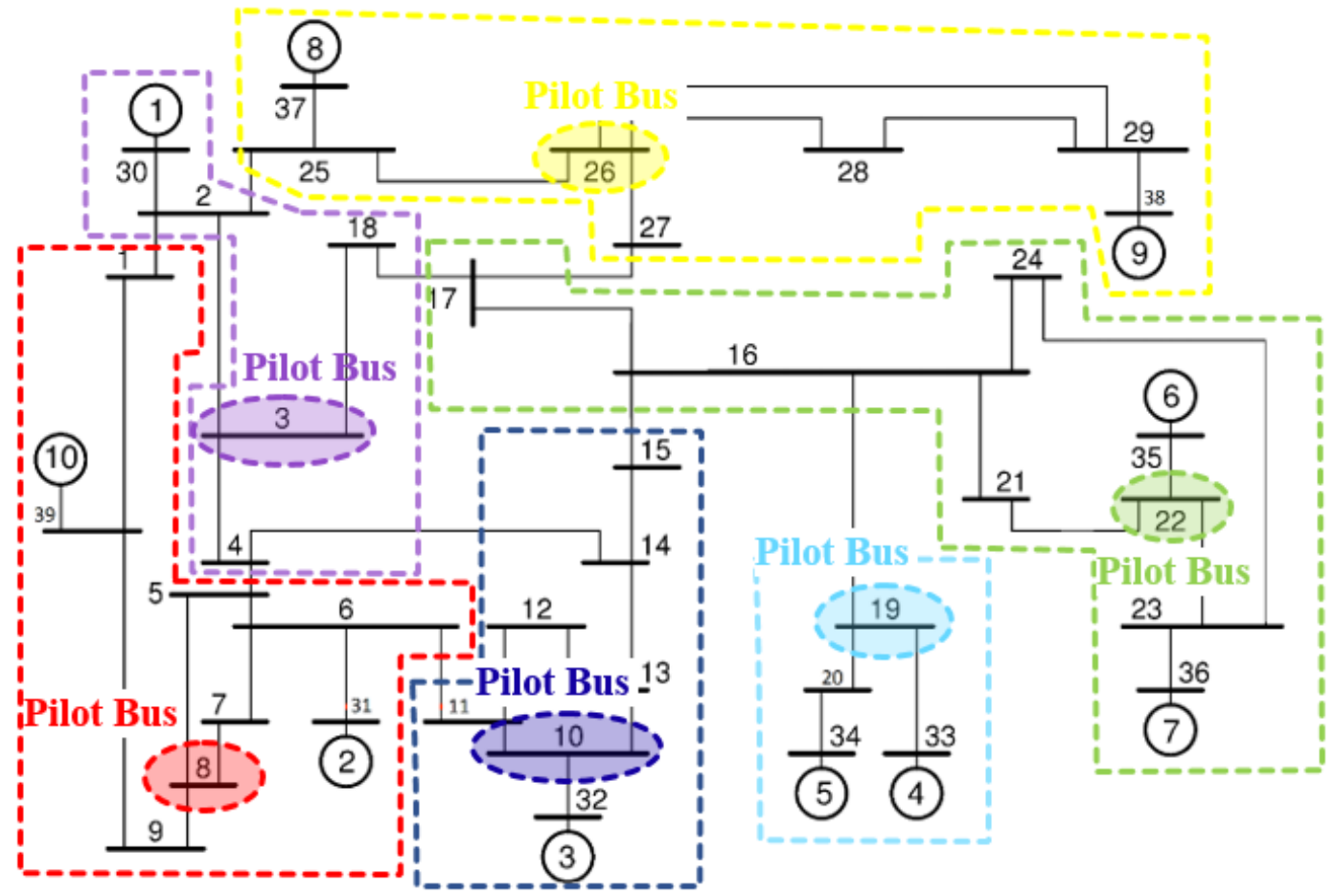

Fig. 3.11. Selected Pilot Buses in three VCAs (39-Bus Power System)

\subsubsection{Performance Comparison}

The performance of the presented community detection algorithm (based on FastGN-Algorithm), Iqbal [67], was compared with the community detection algorithm presented Zhao [60] which is based on original GN-algorithm (proposed by Newman [56]). Promising results were obtained in terms of computation time needed. The average computation time in three cases (9-bus, 39-bus and 118-bus) for both algorithms is shown in Fig. 3.12. The community detection algorithm presented in this research gives similar results (because we are not checking all combinations in each iteration) but the advantage is the reduced computational time required to discover communities (i.e. $4 \%, 73 \%$ and $94 \%$ 
time-reduction for 9-bus, 39-bus and 118-bus power systems respectively as shown in Fig. 3.12). It is evident from the results that our algorithm is better in terms of computational time especially for large power systems.
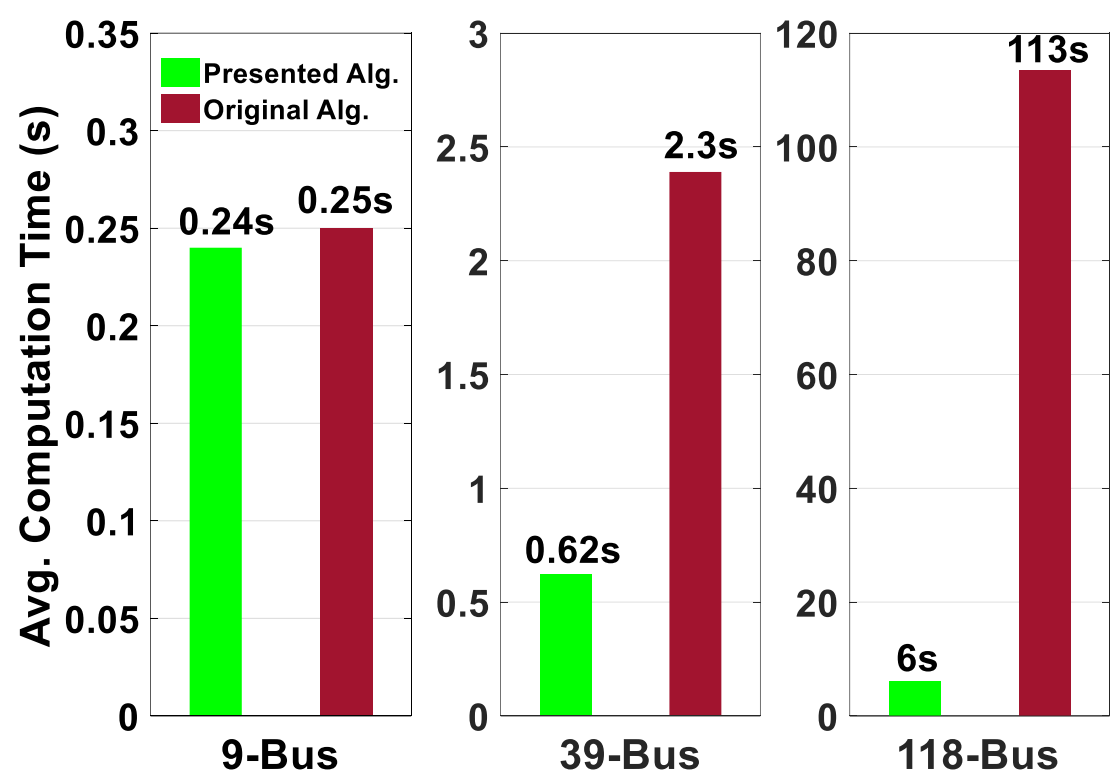

Fig. 3.12. Average Computational Time Comparison 


\section{Chapter 4}

\section{Optimal Voltage Control using SVD}

\subsection{Introduction}

After decomposing a power system into voltage control areas (VCAs) and selecting pilot buses in each area as a representative of the voltage profile of the whole area, the next step is to control the voltage magnitudes of the pilot buses. The idea behind secondary voltage control using pilot points is that when we divide a power system into small areas (i.e. VCAs) based on $V$ - $Q$ sensitivity, then all buses in one VCA have similar behavior in terms of voltage variations when this VCA is subjected to an external disturbance (i.e. reactive power injection). Since a pilot bus is selected such that it has highest sensitivity to reactive power injection in its corresponding VCA compared to other buses in that VCA, if the voltage of this pilot bus is kept constant before and after disturbance, then the voltages of all other buses would vary very little. So, we can say that the pilot bus is a representative of the voltage profile of the whole VCA and the voltage profile of the corresponding VCA can be controlled by controlling only the voltage of one bus (hence the name pilot bus).

There are many different approaches which can be used to control the voltages of the pilot buses depending on different objectives under consideration. In this chapter, an optimal voltage control algorithm is proposed to obtain new voltage set-points for PV buses by maximizing the effect of change in input (i.e. $\Delta \mathrm{V}_{\mathrm{PV}}$ ) on change in output (i.e. $\Delta \mathrm{V}_{\mathrm{PQ}}$ ) using the FDLF jacobian matrix, so that we can have maximum reactive reserve and minimum shift of the controls. The remainder of the chapter is organized as follows: The 


\section{CHAPTER 4. OPTIMAL VOLTAGE CONTROL USING SVD}

proposed optimal voltage control approach is formulated in Section 4.2. Section 4.2.4 describes all steps involved in the proposed voltage control algorithm through a control flow diagram. Section 4.3 discusses implementation and simulation results of the proposed voltage control approach through case studies. Performance comparison with sensitivity based voltage control approach is presented in Section 4.3.3.

\subsection{Problem Formulation}

\subsubsection{Eigenvalues and Eigenvectors}

Linear systems can be represented mathematically by linear matrix equations. Consider a linear system represented by a $2 \times 2$ matrix $\mathbf{A}$. It takes $\mathbf{x}$ as input vector and transforms it linearly into $\mathbf{y}$ as output vector as shown in Fig. 4.1, where $\mathbf{y}=\mathbf{A x}$. When $\mathbf{A}$ operates on $\mathbf{x}$, it changes its magnitude as well as direction.
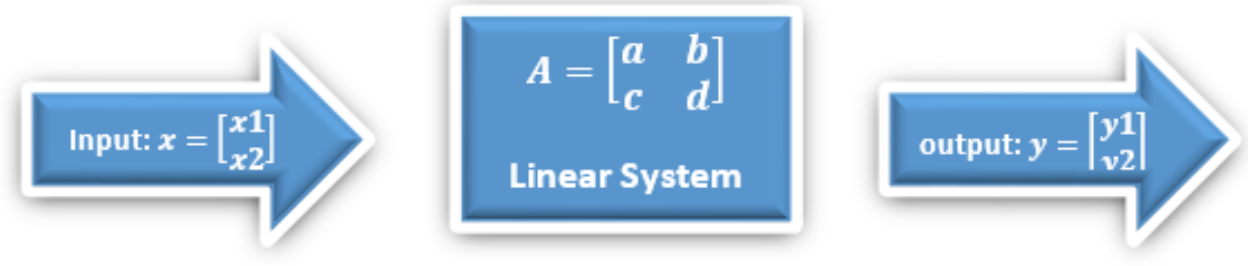

Fig. 4.1: Manipulation of a vector with a linear system

However, there are some special vectors which, when applied on the system, do not change their direction but only magnitude is affected i.e. they are stretched or squeezed only. These special vectors are called eigenvectors $\left(\boldsymbol{v}_{\lambda_{i}}\right)$ of the matrix $\mathbf{A}$ and the amount of stretch or squeeze is called its eigenvalue $\left(\lambda_{\mathrm{i}}\right)$. In other words, when we apply $\boldsymbol{v}_{\lambda_{i}}$ (an eigenvector of $\mathbf{A}$ ) to $\mathbf{A}$, we get another vector whose direction is the same as $v_{\lambda_{i}}$ but its magnitude is scaled by some factor $\left(\lambda_{\mathrm{i}}\right)$ which is called eigenvalue i.e. eigenvectors are those vectors which do not knock off their span when operated by the linear system A, Lay [68], Strang [69].

$$
\text { i.e. } \quad A v_{\lambda_{i}}=\lambda_{i} v_{\lambda_{i}}
$$

Since an eigenvector of a matrix (e.g. A) only changes its magnitude when multiplied by the matrix, it indicates a direction in which $\mathbf{A}$ would have maximum effect on the input, 


\section{CHAPTER 4. OPTIMAL VOLTAGE CONTROL USING SVD}

Tilekar [70]. In other words, any change in input which lies in the span of the eigenvector $\boldsymbol{v}_{\lambda_{m}}$ (the eigenvector corresponding to the highest eigenvalue, $\lambda_{\mathrm{m}}$, of $\mathbf{A}$ ), would bring maximum change in the output i.e.

$$
\begin{gathered}
\text { If } \\
\text { Then } \quad \Delta y=a \Delta x=a v_{\lambda_{m}} A=a \lambda_{m} A
\end{gathered}
$$

where $\alpha$ is a scalar. Now if we consider all the vectors $\left(\boldsymbol{x}_{i}\right)$ having same magnitudes (say ' $x$ ') around an origin $(O)$, then they can be represented by a circle of radius ' $x$ ' with ' $O$ ' as its center as shown in Fig. 4.2.

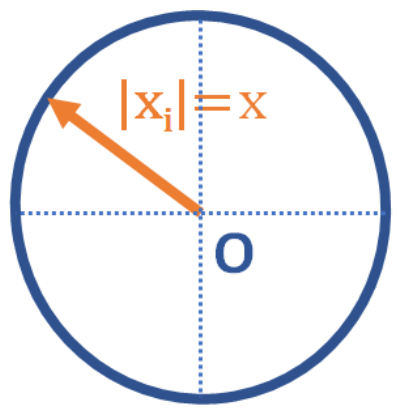

Fig. 4.2: All vectors $\boldsymbol{x}_{i}$ around origin 'O' having same magnitude ' $x$ '

When all these vectors are manipulated by the matrix $\mathbf{A}$, then all the corresponding output vectors $\left(\boldsymbol{y}_{i}\right)$ form a shape as shown in Fig. 4.3.

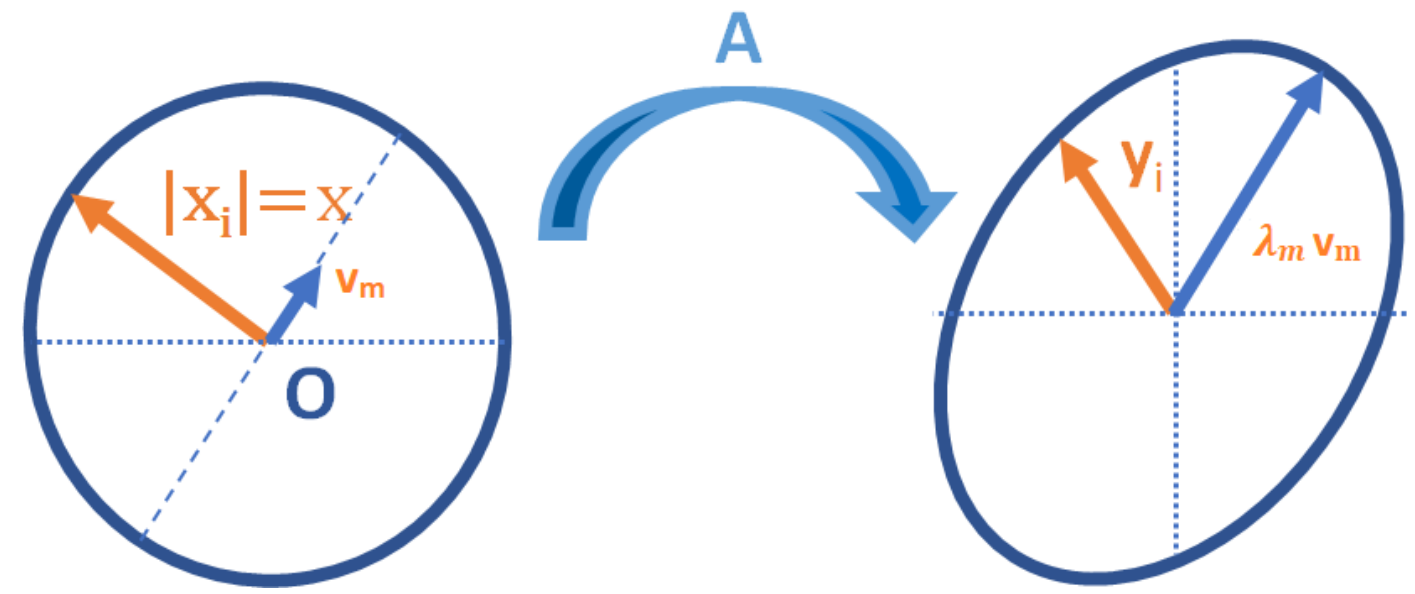

Fig. 4.3: Manipulation of input vectors $\boldsymbol{x}_{i}$ with $\boldsymbol{A}$ and corresponding output vectors $\boldsymbol{y}_{i}$ 
From Fig. 4.3, it is clear that magnitude of the output vector is maximized when input $\boldsymbol{x}_{\mathrm{i}}$ lies in the span of $\mathbf{v} \mathbf{m}$ where $\mathbf{v m}$ is the eigenvector corresponding to highest eigenvalue $\left(\lambda_{\boldsymbol{m}}\right)$ of $\mathbf{A}$.

\subsubsection{Singular Value Decomposition}

The same concept can also be applied to singular vectors of the matrix A Lay [71], Strang [72]. The singular value decomposition (SVD) of a matrix $\mathbf{A}$ is defined as in (1)

$$
A=U \sum V^{T}
$$

where

$$
\begin{gathered}
\boldsymbol{U}=\left[\begin{array}{lll}
\boldsymbol{u}_{1} & \boldsymbol{u}_{2} & \ldots .
\end{array}\right] \\
\boldsymbol{V}^{T}=\left[\begin{array}{c}
\boldsymbol{v}_{1}^{T} \\
\boldsymbol{v}_{2}^{T} \\
\vdots
\end{array}\right] \\
\sum=\left[\begin{array}{ccc}
\sigma_{1} & \mathbf{0} & \mathbf{0} \\
\mathbf{0} & \sigma_{2} & \mathbf{0} \\
\mathbf{0} & \mathbf{0} & \ddots
\end{array}\right]
\end{gathered}
$$

The diagonal elements of the matrix $\sum$ are the singular values of the matrix $\mathbf{A}$. The columns $\left(\boldsymbol{u}_{i}\right)$ of the matrix $\mathbf{U}$ are called the left singular vectors of $\mathbf{A}$ (eigenvectors of $\mathbf{A} \mathbf{A}^{\mathrm{T}}$ ) and rows $\left(\boldsymbol{v}_{i}^{T}\right)$ of the matrix $\mathbf{V}^{\mathrm{T}}$ are called the right singular vectors of $\mathbf{A}$ (eigenvectors of $\mathbf{A}^{\mathrm{T}} \mathbf{A}$ ). The eigenvalues of $\mathbf{A A}^{\mathrm{T}}$ are the singular values of $\mathbf{A}$.

$$
\begin{aligned}
\text { If } & \boldsymbol{x}=a \boldsymbol{v}_{\boldsymbol{i}} \\
\text { Then } \quad \boldsymbol{y}=\boldsymbol{A} \boldsymbol{x} & =a \sigma_{\boldsymbol{i}} \boldsymbol{u}_{\boldsymbol{i}}
\end{aligned}
$$

The output vector $\mathbf{y}$ is maximized when input $\boldsymbol{x}_{\mathrm{i}}$ lies in the span of $\boldsymbol{v}_{\mathbf{1}}$ where $\boldsymbol{v}_{\mathbf{1}}$ is the right singular vector corresponding to highest singular value $\left(\sigma_{\mathbf{1}}\right)$ of $\mathbf{A}$. 


\subsubsection{Optimal Voltage Control}

Using fast decoupled load flow assumptions, the changes in network bus voltage magnitudes can be approximated by (4.2)

$$
\Delta V=-[B "]^{-1} \Delta Q=S^{V Q} \Delta Q
$$

$\mathbf{S}^{\mathrm{VQ}}$ is $Q-V$ sensitivity matrix of the network. By decomposing the vector $\boldsymbol{\Delta V}$ into voltagecontrolled/PV buses and load/PQ buses, we can re-write (4.2) as

$$
\left[\begin{array}{l}
\Delta V_{P V} \\
\Delta V_{P Q}
\end{array}\right]=\left[\begin{array}{ll}
s_{Q_{P V}}^{V_{P V}} & S_{Q_{P Q}}^{V_{P V}} \\
s_{Q_{P Q}}^{V_{P Q}} & S_{Q_{P Q}}^{V_{P Q}}
\end{array}\right]\left[\begin{array}{l}
\Delta Q_{P V} \\
\Delta Q_{P Q}
\end{array}\right]
$$

where

$$
\begin{aligned}
& S_{Q_{P V}}^{V_{P V}}=\text { sensitivity of } \Delta V_{\mathrm{PV}} \text { w.r.t } \Delta \mathrm{Q}_{\mathrm{PV}} \\
& S_{Q_{P Q}}^{V_{P V}}=\text { sensitivity of } \Delta V_{\mathrm{PV}} \text { w.r.t } \Delta \mathrm{Q}_{\mathrm{PQ}} \\
& S_{Q_{P V}}^{V_{P Q}}=\text { sensitivity of } \Delta \mathrm{V}_{\mathrm{PQ}} \text { w.r.t } \Delta \mathrm{Q}_{\mathrm{PV}} \\
& S_{Q_{P Q}}^{V_{P Q}}=\text { sensitivity of } \Delta \mathrm{V}_{\mathrm{PQ}} \text { w.r.t } \Delta \mathrm{Q}_{\mathrm{PQ}}
\end{aligned}
$$

Using (4.3), we can write two sets of equations as

$$
\begin{aligned}
& \Delta V_{P V}=S_{Q_{P V}}^{V_{P V}} \Delta Q_{P V}+S_{Q_{P Q}}^{V_{P V}} \Delta Q_{P Q} \\
& \Delta V_{P Q}=S_{Q_{P V}}^{V_{P Q}} \Delta Q_{P V}+S_{Q_{P Q}}^{V_{P Q}} \Delta Q_{P Q}
\end{aligned}
$$

By separating $\Delta \boldsymbol{Q}_{\boldsymbol{P V}}$ from (4.4), we get

$$
\Delta Q_{P V}=\left[S_{Q_{P V}}^{V_{P V}}\right]^{-1} \Delta V_{P V}-\left[S_{Q_{P V}}^{V_{P V}}\right]^{-1} S_{Q_{P Q}}^{V_{P V}} \Delta Q_{P Q}
$$

Putting (4.6) in (4.5), we obtain

$$
\begin{gathered}
\Delta V_{P Q}=S_{Q_{P V}}^{V_{P Q}}\left[S_{Q_{P V}}^{V_{P V}}\right]^{-1} \Delta V_{P V}+\left(S_{Q_{P Q}}^{V_{P Q}}-S_{Q_{P V}}^{V_{P Q}}\left[S_{Q_{P V}}^{V_{P V}}\right]^{-1} S_{Q_{P Q}}^{V_{P V}}\right) \Delta Q_{P Q} \\
\Delta V_{P Q}=S_{V_{P V}}^{V_{P Q}} \Delta V_{P V}+D
\end{gathered}
$$


where

$$
\begin{aligned}
& S_{V_{P V}}^{V_{P Q}}=S_{Q_{P V}}^{V_{P Q}}\left[S_{Q_{P V}}^{V_{P V}}\right]^{-1} \\
& D=\left(S_{Q_{P Q}}^{V_{P Q}}-S_{Q_{P V}}^{V_{P Q}}\left[S_{Q_{P V}}^{V_{P V}}\right]^{-1} S_{Q_{P Q}}^{V_{P V}}\right) \Delta Q_{P Q}
\end{aligned}
$$

Here $\mathbf{D}$ is considered as a disturbance because we have no control over reactive power demand. So, we can approximate (4.8) by neglecting $\mathbf{D}$ as

$$
\Delta V_{P Q} \simeq S_{V_{P V}}^{V_{P Q}} \Delta V_{P V}(\text { neglecting } D)
$$

Equation (4.9) gives relation between voltages of PQ buses and PV buses. So, we can use (4.9) to control PQ-bus voltages by adjusting PV-bus voltages appropriately.

The objective of the controller is to find optimal input i.e. $\Delta \mathbf{V}_{\mathrm{PV}}$ that would have maximum effect on the output i.e. $\Delta \mathbf{V}_{\mathrm{PQ}}$ (using weighted $\mathrm{L}_{2}$ norm of the output) so that we can have maximum reactive power reserve and minimum shift of the controls. The objective function $\mathrm{J}$ can be described by (4.10)

$$
\begin{aligned}
& \max _{\Delta \boldsymbol{V}_{P V}} J=\Delta \boldsymbol{V}_{P Q}^{T} \boldsymbol{M} \boldsymbol{\Delta} \boldsymbol{V}_{P Q} \\
& \text { s.t. } 0.9<\left[\begin{array}{c}
\Delta V_{P V} \\
\Delta V_{P Q}
\end{array}\right]+\left[\begin{array}{l}
V_{P V}^{\text {old }} \\
V_{P Q}^{\text {old }}
\end{array}\right]<1.1
\end{aligned}
$$

Where $\mathbf{M}$ is weight matrix to select the load bus we want to control (i.e. pilot bus). Using (4.9), J can be approximated as

$$
J=\Delta \boldsymbol{V}_{P Q}^{T} \boldsymbol{M} \boldsymbol{\Delta} \boldsymbol{V}_{P Q}=\left(\boldsymbol{S}_{\boldsymbol{V}_{P V}}^{V_{P Q}} \boldsymbol{\Delta} \boldsymbol{V}_{\boldsymbol{P V}}\right)^{T} \boldsymbol{M}\left(\boldsymbol{S}_{\boldsymbol{V}_{P V} \boldsymbol{V}}^{V_{P Q}} \boldsymbol{\Delta} \boldsymbol{V}_{\boldsymbol{P V}}\right)=\boldsymbol{\Delta} \mathbf{V}_{P V}^{T} \boldsymbol{N} \boldsymbol{\Delta} \mathbf{V}_{P V}
$$

Where $\boldsymbol{N}=\left[s_{Q_{P V}}^{V_{P V}}\right]^{-T}\left[s_{Q_{P V}}^{V_{P Q}}\right]^{T} M\left[s_{Q_{P V}}^{V_{P Q}}\right]\left[s_{Q_{P V} V_{P V}}^{-1}\right]^{-1}$. Objective function J would be maximized if we select $\Delta \mathbf{V}_{\mathrm{PV}}$ in the span of the left singular vector of $\mathbf{N}$ which corresponds to its highest singular value (i.e. $\sigma_{1}$ ). Using singular value decomposition of $\mathbf{N}\left(\right.$ i.e. $\mathbf{N}=\mathbf{U} \sum \mathbf{V}^{\mathrm{T}}$ ), (4.11) can be modified as in (4.12)

$$
J=\Delta \mathbf{V}_{P V}^{T} \mathbf{U} \sum \boldsymbol{V}^{T} \Delta \mathbf{V}_{P V}
$$


If $\Delta \mathbf{V}_{\mathrm{PV}}=\alpha \mathbf{v}_{1}$, where $\mathrm{v}_{1}$ is the right singular vector corresponding to the highest singular value of $\mathbf{N}$, then performance index $\mathbf{J}$ would be maximized and (4.12) would be modified as in (4.13), Talha [73-74]

$$
J_{\max }=\alpha^{2} \sigma_{1}
$$

Scalar constant $\alpha$ is the design parameter in this control problem which would give required change in voltage of the pilot bus being controlled. In this approach, we are controlling one pilot bus in every voltage control area (VCA). By selecting $\mathbf{M}$ appropriately (depending on index of the pilot bus in VCA), we can obtain using (4.10) and (4.13)

$$
\Delta \boldsymbol{V}_{\boldsymbol{P Q}}^{\boldsymbol{T}} \boldsymbol{M} \boldsymbol{\Delta} \boldsymbol{V}_{\boldsymbol{P Q}}=\Delta V_{\text {pilot }}^{2}=J_{\max }=\alpha^{2} \sigma_{1}
$$

Design parameter $\alpha$ can be calculated using (4.15)

$$
\alpha=\frac{\Delta V_{\text {pilot }}}{\sqrt{\sigma_{1}}}
$$

\subsubsection{Secondary Voltage Control}

After decomposing the whole power system into voltage control areas (VCAs), the controller in each VCA would be responsible to keep the corresponding pilot bus voltage constant in decentralized manner by re-adjusting the reference voltages for the exciters (i.e. primary voltage controller - PVC) at generator/PV buses. A typical architecture of secondary voltage control (SVC) is shown in Fig. 4.4.

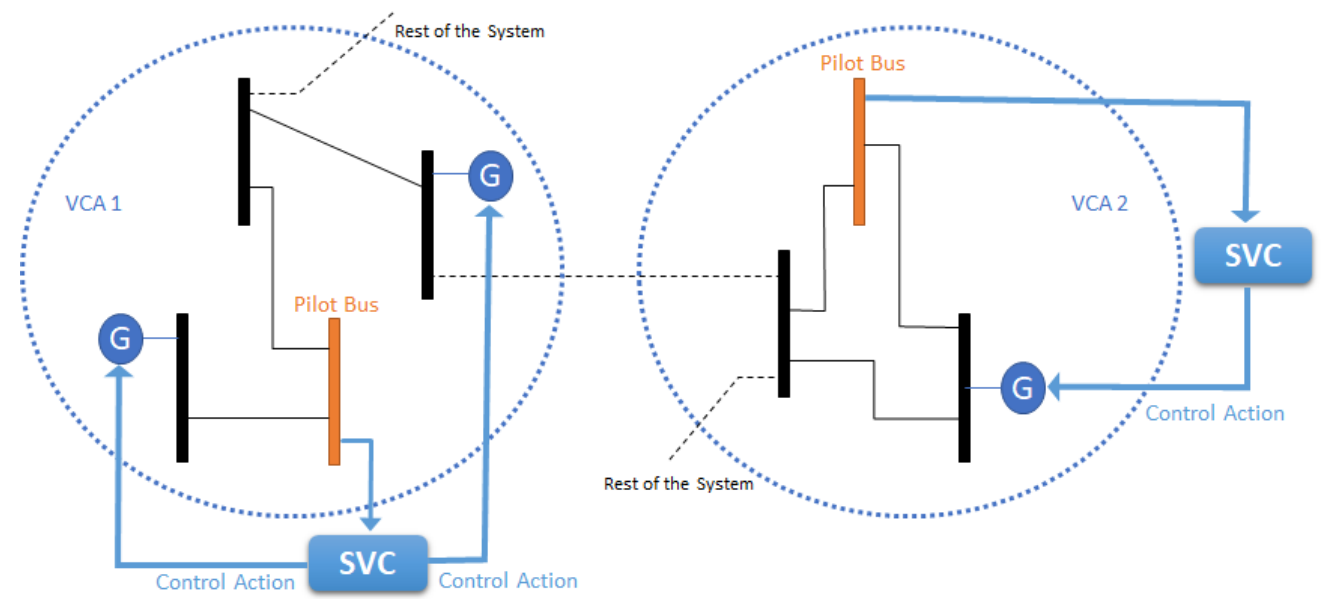

Fig. 4.4: Decentralized Secondary Voltage Control (SVC) Architecture 


\section{CHAPTER 4. OPTIMAL VOLTAGE CONTROL USING SVD}

As shown in Fig. 4.4, each SVC is responsible to control its corresponding pilot bus voltage by adjusting the reference voltages of generator buses in its own VCA. The objective of an SVC is to find the optimal values (i.e. $\Delta \mathbf{V}_{\mathrm{PV}}=\alpha \mathbf{v}_{1}$ ) for reference voltages of all generator buses in its VCA using (4.15) such that pilot bus voltage comes back to its predefined value after disturbance(s).

\subsubsection{SVC Algorithm}

Let's consider a VCA. The predefined value for the pilot bus in this VCA is $V_{\text {pilot }}^{\text {old }}$. Then the proposed optimal secondary voltage control algorithm for this VCA can be described by following steps:

6 Initialize $\Delta \boldsymbol{V}_{\boldsymbol{P V}}=\mathbf{0}$ (zero vector) and calculate $\Delta V_{\text {pilot }}=V_{\text {pilot }}^{\text {new }}-V_{\text {pilot }}^{\text {old }}$.

7 IF: $\left|\Delta V_{\text {pilot }}\right|<\varepsilon$, where $\varepsilon$ is a small number representing allowable difference between $V_{\text {pilot }}^{\text {old }}$ and $V_{\text {pilot }}^{\text {new }}$, then return to step 1.

8 ELSE: compute $\alpha$ using (4.15) which would bring back pilot bus voltage within allowable limits.

9 Calculate $\boldsymbol{\Delta} \boldsymbol{V}_{\boldsymbol{P V}}$ using (4.16) which would give required change in PV-bus voltages.

$$
\Delta \boldsymbol{V}_{\boldsymbol{P V}}=\alpha \mathbf{v}_{1}
$$

10 Update voltages of the voltage-controlled buses $\left(\boldsymbol{V}_{\boldsymbol{P V}, \text { new }}\right)$ using (4.17) and return to step 1.

$$
\mathrm{V}_{P V, \text { new }}=\mathrm{V}_{P V, \text { old }}+\Delta \mathrm{V}_{P V}
$$

The control flow diagram of the proposed voltage control algorithm for each VCA is shown in Fig. 4.5. 


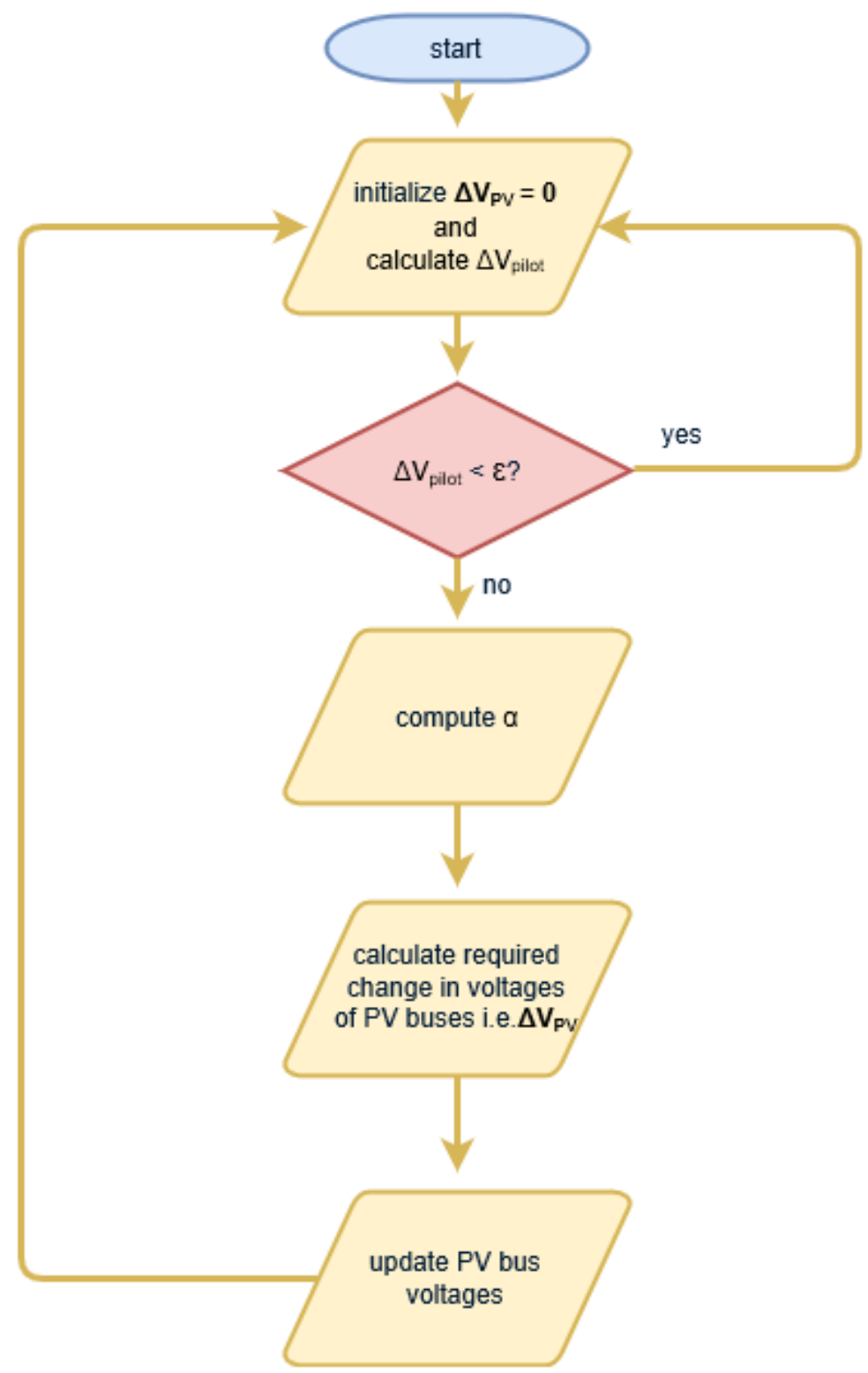

Fig. 4.5: Control Flow diagram for proposed SVC algorithm

\subsection{Case Studies and Simulation Results}

In this section, the proposed optimal voltage control algorithm would be applied on two power systems that we studied in chapter 3 i.e. 9-bus and 39-bus power system for which we obtained voltage control areas and pilot points, Zimmerman [75]. In every case, we randomly added some disturbances (inductive or capacitive) into the original system to 
make pilot bus voltage(s) go out of limits and then applied this control algorithm to bring them back within allowable limits. All simulations results were obtained using MATLAB and MATPOWER 6.0, Zimmerman [76].

\subsubsection{9-Bus Power Network}

This system has three (bus 1-3) voltage controlled buses (including slack bus) and six (bus 4-9) load buses. The active power demand is $315 \mathrm{MW}$ while reactive power demand is 115 MVAR. The single line diagram of the system is shown in Fig. 4.6 along with the reactive power demands under normal conditions. All voltages are within limits ( 0.9 to 1.1 pu) under normal operating conditions as shown in Table 4.1.

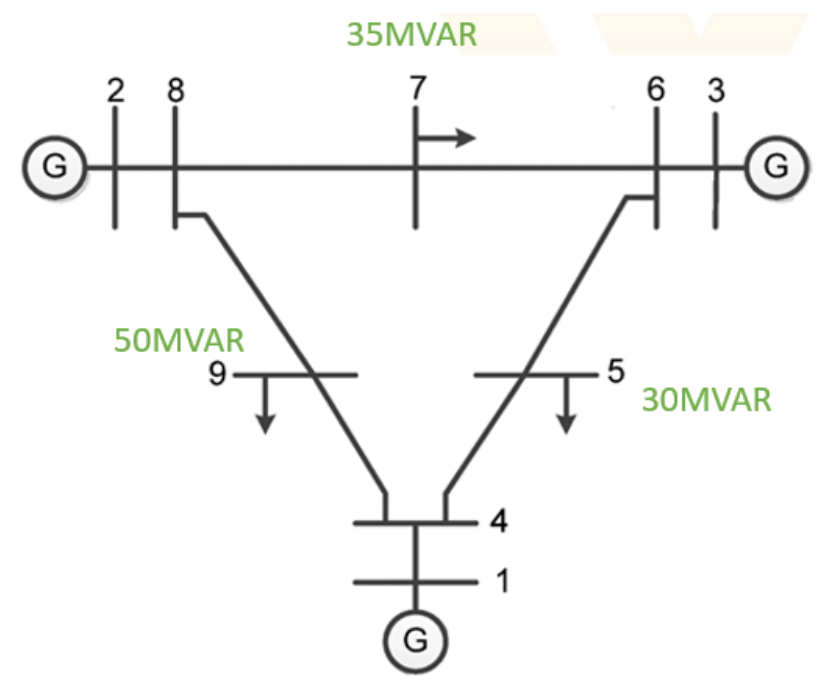

Fig. 4.6. Reactive power demand under normal conditions (9-Bus Power System)

The voltage control areas and pilot points for this system obtained in chapter 3 are shown in Fig. 4.7. The system was decomposed into three voltage control areas each having three buses which indicates that it's a highly random and symmetrical network and it's also clear from physical topology of the network. 


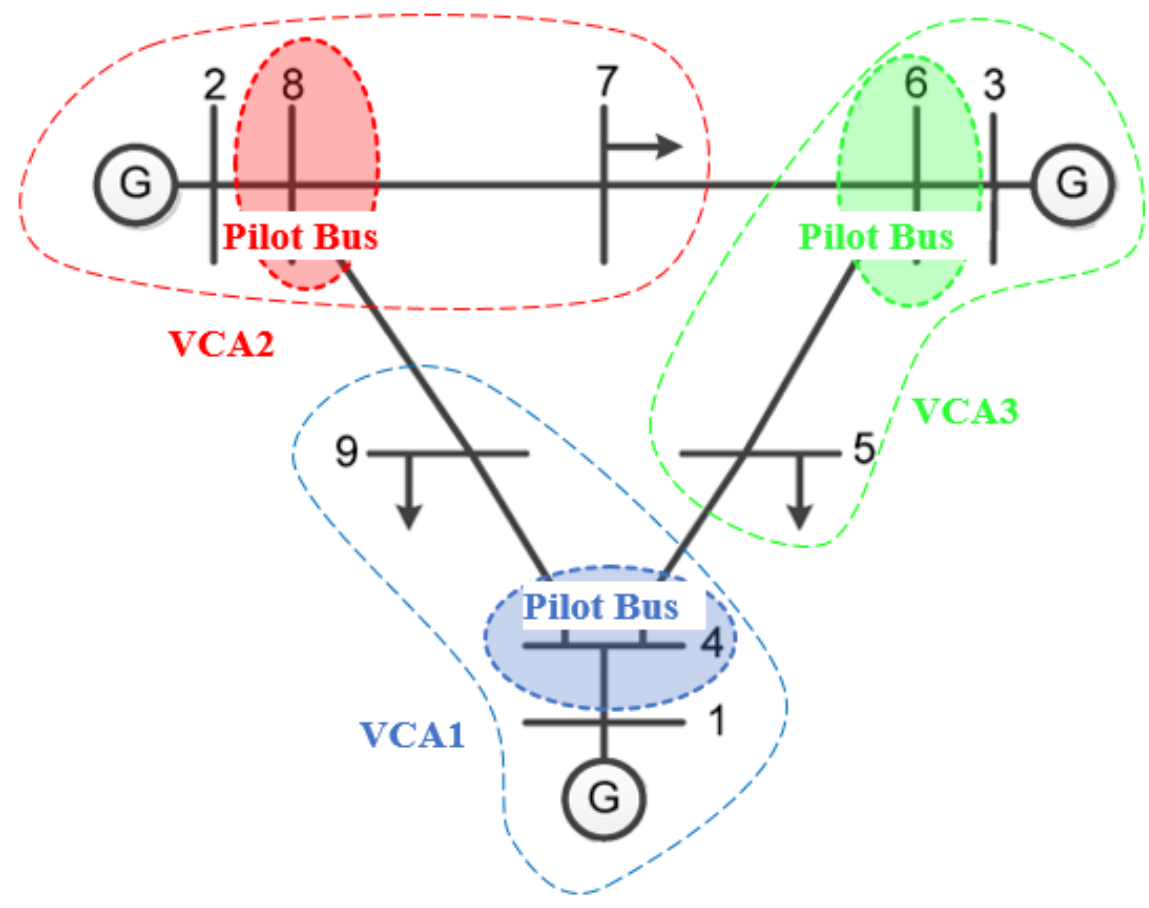

Fig. 4.7. Obtained VCAs and Pilot Buses (9-Bus Power System)

Table 4.1 Voltages under Normal Operating Conditions (9-Bus Power System)

\begin{tabular}{|c|c|c|c|}
\hline Bus \# & Voltage (pu) & Bus \# & Voltage (pu) \\
\hline $\mathbf{1}$ & 1 & 6 & 1 \\
\hline $\mathbf{2}$ & 1 & $\mathbf{7}$ & 0.985 \\
$\mathbf{3}$ & 1 & $\mathbf{8}$ & $\mathbf{0 . 9 9 6}$ \\
\hline 4 & 0.987 & $\mathbf{9}$ & 0.957 \\
\hline $\mathbf{5}$ & 0.975 & & \\
\hline
\end{tabular}

\subsubsection{Subjecting to Perturbations}

We can consider each VCA as a separate subsystem and SVC in each area would be responsible to control the voltage of its own pilot bus. The data for each VCA is given in Table 4.2. 
CHAPTER 4. OPTIMAL VOLTAGE CONTROL USING SVD

Table 4.2 Data for each Voltage Control Area (9-Bus Power System)

\begin{tabular}{|c|c|c|c|}
\hline Area & VCA1 & VCA2 & VCA3 \\
\hline Buses in area & $1,4,9$ & $2,7,8$ & $3,5,6$ \\
\hline $\begin{array}{c}\text { Relation } \\
\text { between } \Delta \mathbf{V}_{\mathbf{P Q}} \\
\text { and } \Delta \mathbf{V}_{\mathbf{P V}}\end{array}$ & {$\left[\begin{array}{l}\Delta V_{4} \\
\Delta V_{9}\end{array}\right]=\left[\begin{array}{l}0.56 \\
0.38\end{array}\right] \Delta V_{1}$} & {$\left[\begin{array}{l}\Delta V_{7} \\
\Delta V_{8}\end{array}\right]=\left[\begin{array}{l}0.35 \\
0.59\end{array}\right] \Delta V_{2}$} & {$\left[\begin{array}{c}\Delta V_{5} \\
\Delta V_{6}\end{array}\right]=\left[\begin{array}{l}0.20 \\
0.57\end{array}\right] \Delta V_{3}$} \\
\hline $\begin{array}{c}\text { Pilot bus } \\
\mathbf{V}_{\text {ref }}\end{array}$ & $0.987 \mathrm{pu}$ & $0.996 \mathrm{pu}$ & 6 \\
\hline $\begin{array}{c}\text { Relation } \\
\text { between } \\
\Delta \mathbf{V}_{\text {pilot and }}\end{array}$ & $\Delta V_{4}=0.56 \Delta V_{1}$ & $\Delta V_{8}=0.56 \Delta V_{2}$ & $\Delta V_{6}=0.56 \Delta V_{3}$ \\
$\Delta \mathbf{V}_{\mathbf{P V}}$ & & & \\
\hline $\boldsymbol{\varepsilon}$ & 0.01 & 0.01 & 0.01 \\
\hline \multicolumn{2}{|c}{} \\
\hline
\end{tabular}

\section{Scenario 1 - Single Perturbation:}

First, we added a single perturbation in a VCA to observe decentralized operation of secondary voltage control (SVC) of the VCA.

\section{30MVAR on bus 9 (VCA1)}

All bus voltages before and after adding 30MVAR disturbance (inductive) on bus 9 are shown in Fig. 4.8. 


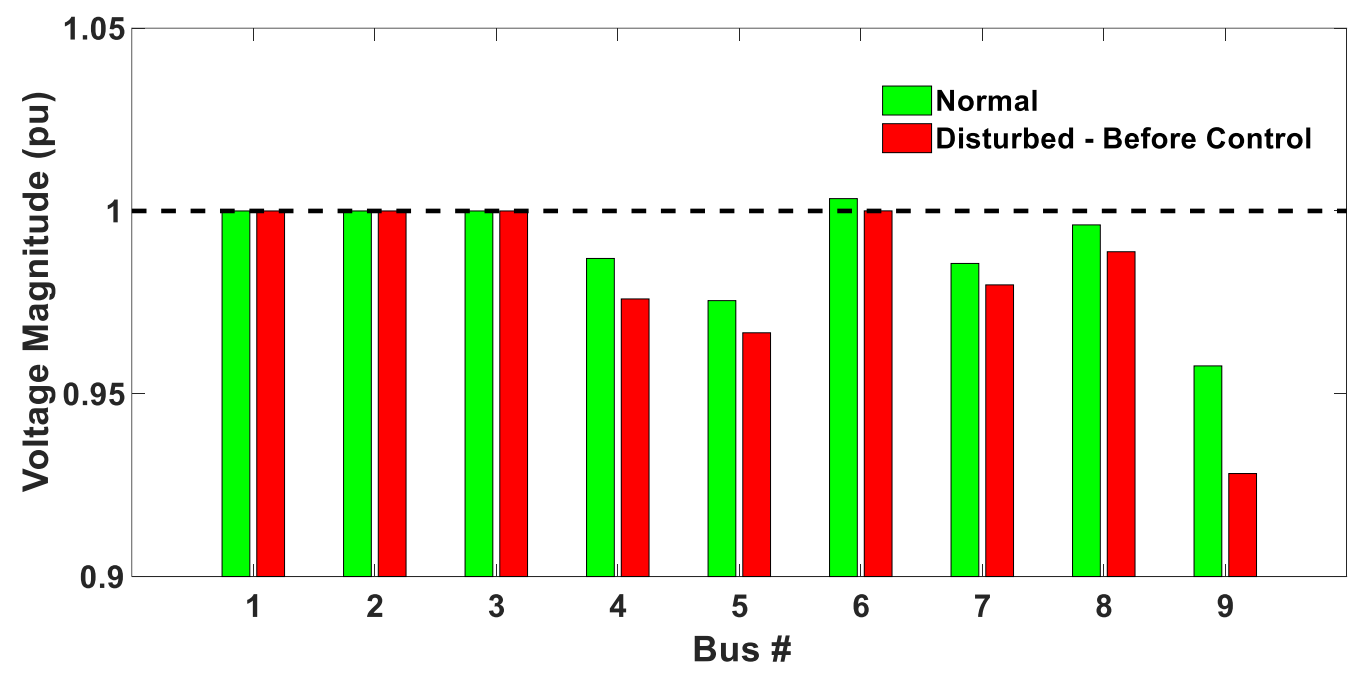

Fig. 4.8. Bus voltages before and after Perturbation in VCAl on bus 9

(9-Bus Power System)

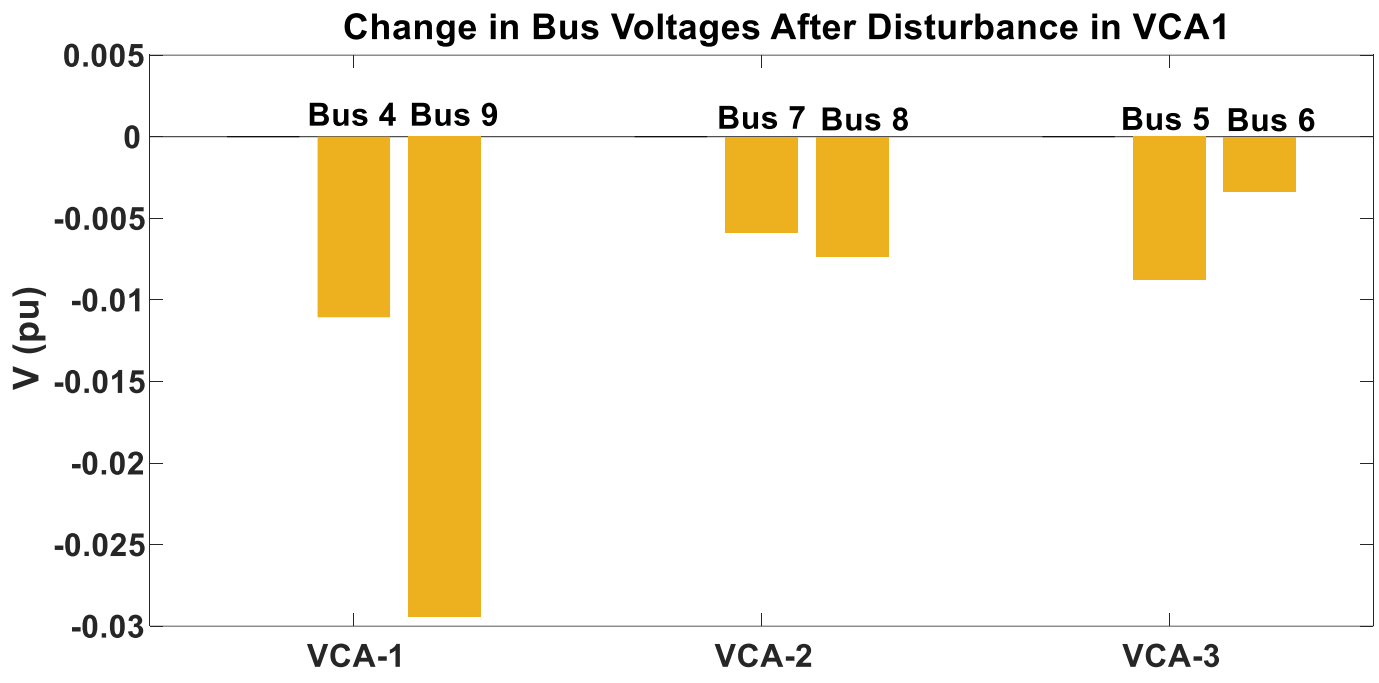

Fig. 4.9. Change in Bus voltages after Perturbation in VCAl on bus 9 (9-Bus Power)

Now if we look at bus voltage changes in each VCA shown in Fig. 4.9, we can observe that area- 1 buses are mostly affected since the disturbance was added in VCA1. It indicates that inter-community $V$ - $Q$ sensitivity is minimized compared to intra-community $V$ - $Q$ sensitivity as claimed in previous chapter where we decomposed the system into VCAs. After applying optimal voltage control, the pilot bus voltages were brought back at reference values (as given in Table 4.2) as shown Fig. 4.10. 
CHAPTER 4. OPTIMAL VOLTAGE CONTROL USING SVD

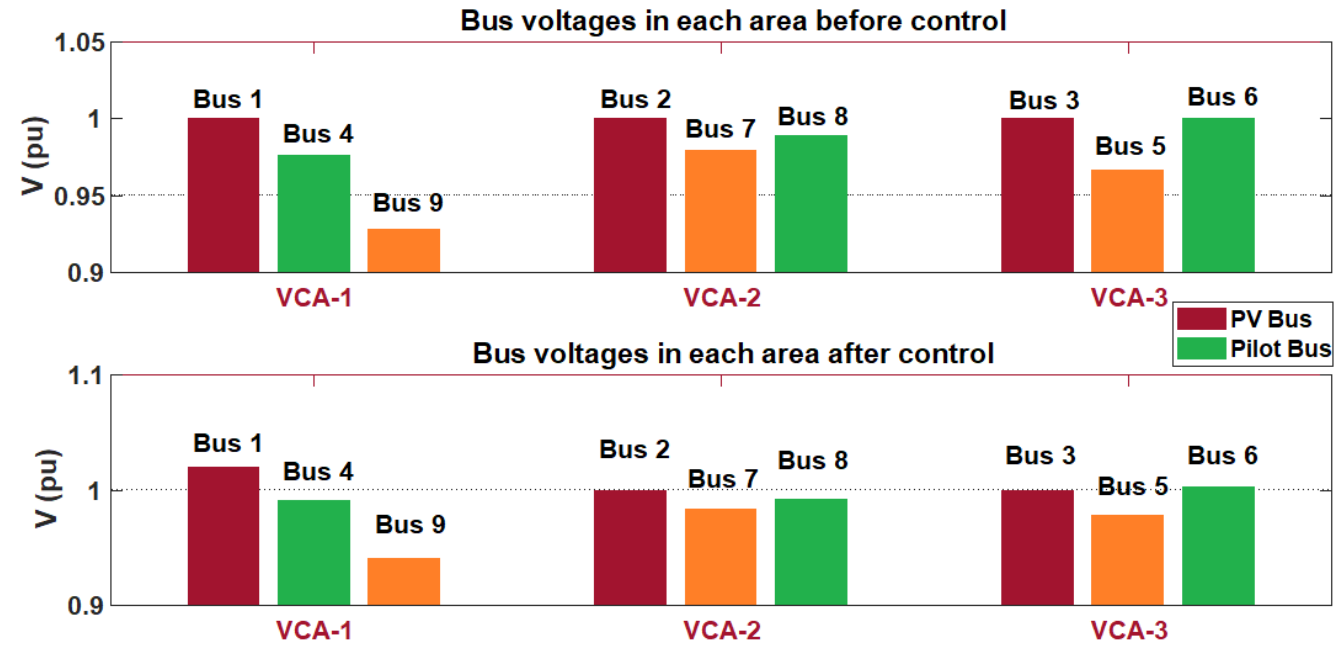

Fig. 4.10. Bus Voltages before and after Control (9-Bus Power System)

By observing the change in generator bus voltages before and after control as shown in Fig. 4.11, it is cleared that only area-1 generator (i.e. Bus-1) is responding to this disturbance which indicates decentralized operation of the system voltage control.

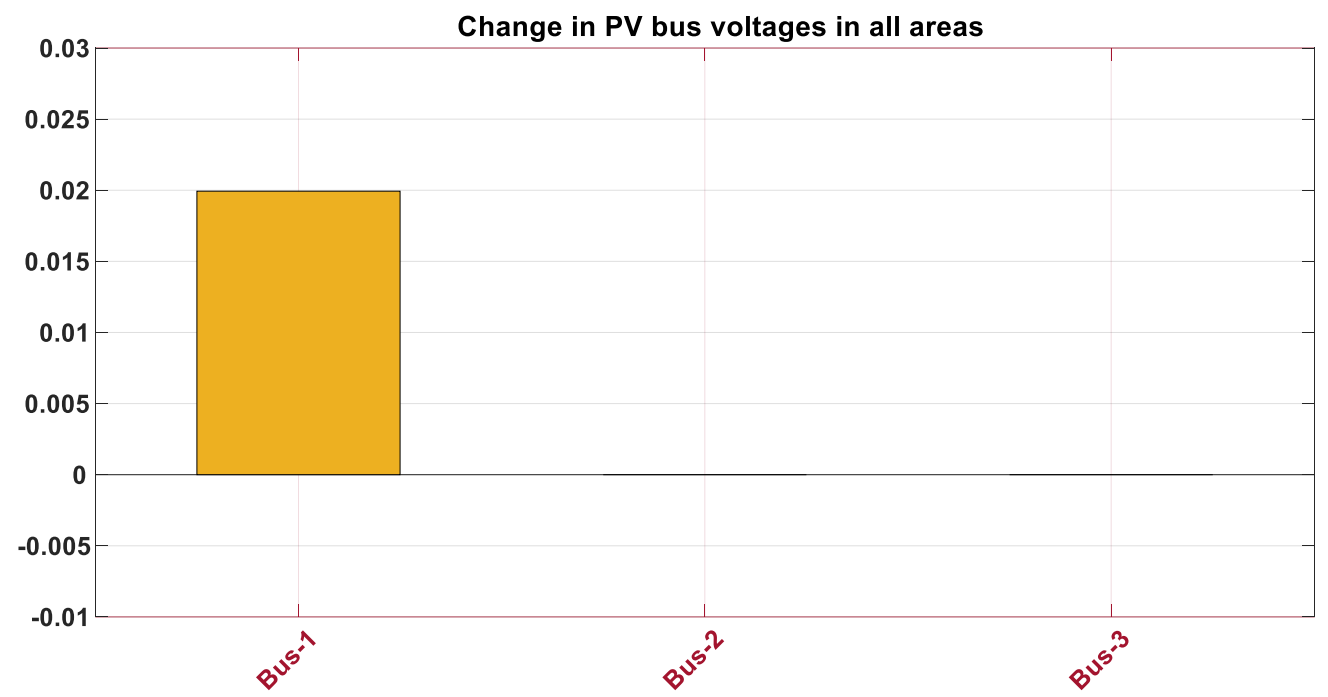

Fig. 4.11. Change in PV-Bus Voltages after Control (9-Bus Power System)

The voltage profile of the whole system before and after control is shown in Fig. 4.12 with the values given in Table 4.3. 
CHAPTER 4. OPTIMAL VOLTAGE CONTROL USING SVD

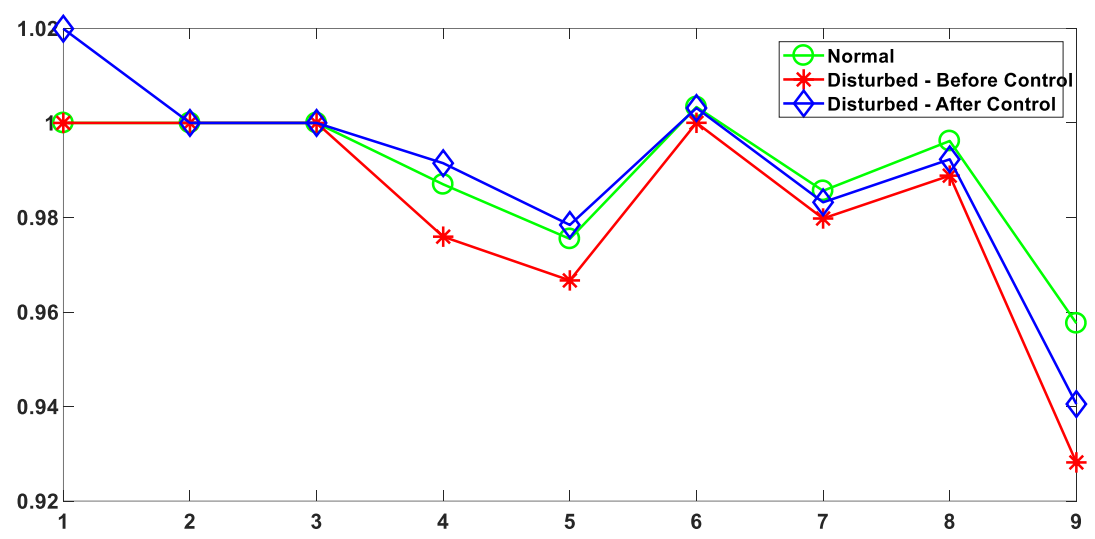

Fig. 4.12. Voltage Profile of the System (9-Bus Power System)

Table 4.3: Bus Voltages in Different Conditions (9-Bus Power System)

\begin{tabular}{|c|c|c|c|}
\hline \multirow{2}{*}{ Bus \# } & \multicolumn{3}{|c|}{ Voltage (pu) } \\
\cline { 2 - 4 } & Normal & $\begin{array}{c}\text { Disturbed } \\
\text { Before Control }\end{array}$ & $\begin{array}{c}\text { Disturbed } \\
\text { After Control }\end{array}$ \\
\hline $\mathbf{1}$ & 1 & 1 & 1.02 \\
\hline $\mathbf{2}$ & 1 & 1 & 1 \\
\hline $\mathbf{3}$ & 1 & 1 & 1 \\
\hline 4 & $\mathbf{0 . 9 8 7}$ & $\mathbf{0 . 9 7 6}$ & $\mathbf{0 . 9 9 1}$ \\
\hline $\mathbf{5}$ & 0.975 & 0.967 & 0.978 \\
\hline 6 & 1 & 1 & 1.003 \\
\hline $\mathbf{7}$ & 0.985 & 0.980 & 0.983 \\
\hline $\mathbf{8}$ & $\mathbf{0 . 9 9 6}$ & $\mathbf{0 . 9 8 9}$ & $\mathbf{0 . 9 9 2}$ \\
\hline $\mathbf{9}$ & 0.957 & 0.928 & 0.941 \\
\hline
\end{tabular}

\section{30MVAR on bus 8 (VCA2)}

All bus voltages before and after adding -30MVAR disturbance (capacitive) on bus 8 are shown in Fig. 4.13. 
CHAPTER 4. OPTIMAL VOLTAGE CONTROL USING SVD

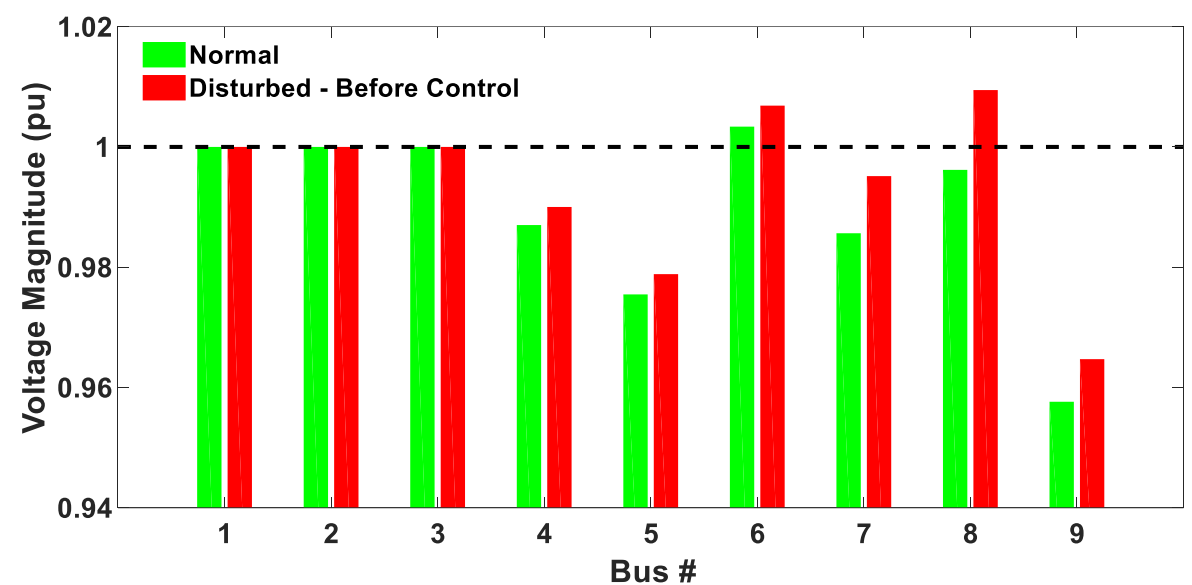

Fig. 4.13. Bus voltages before and after Perturbation in VCA2 on bus 8

(9-Bus Power System)

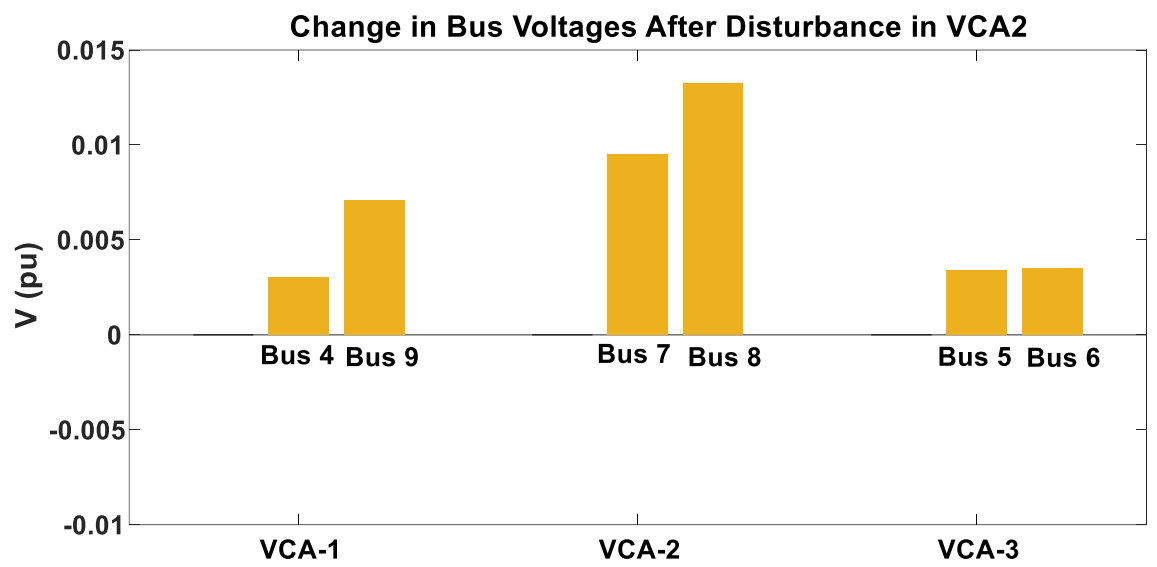

Fig. 4.14. Change in Bus voltages after Perturbation in VCA2 on bus 8 (9-Bus Power System)

Again, if we look at bus voltage changes in each VCA shown in Fig. 4.14, we can observe that area-2 buses are mostly affected since the disturbance was added in VCA2. It again proves that inter-community $V$ - $Q$ sensitivity is minimized compared to intra-community $V-Q$ sensitivity.

After applying voltage control, the pilot bus voltages were brought back at reference values as shown Fig. 4.15. 
CHAPTER 4. OPTIMAL VOLTAGE CONTROL USING SVD
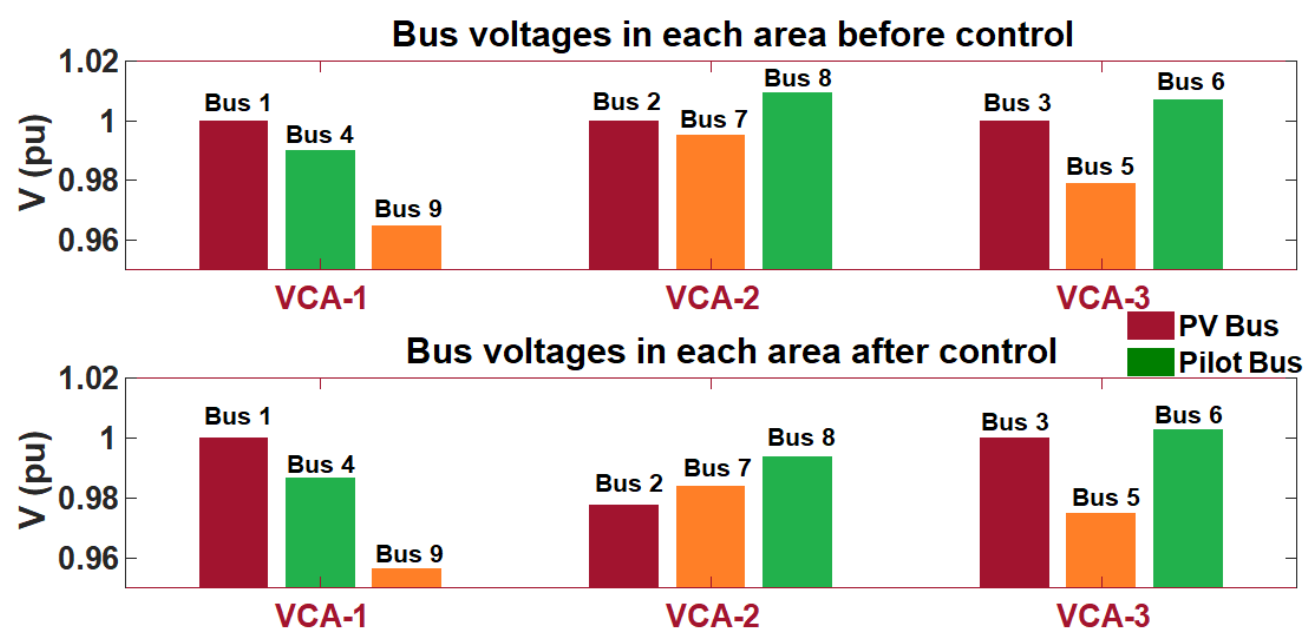

Fig. 4.15. Bus Voltages before and after Control (9-Bus Power System)

By observing the change in generator bus voltages before and after control as shown in Fig. 4.16, it is again cleared that only area-2 generator (i.e. Bus-2) is responding to this disturbance which indicates decentralized operation of voltage controllers.

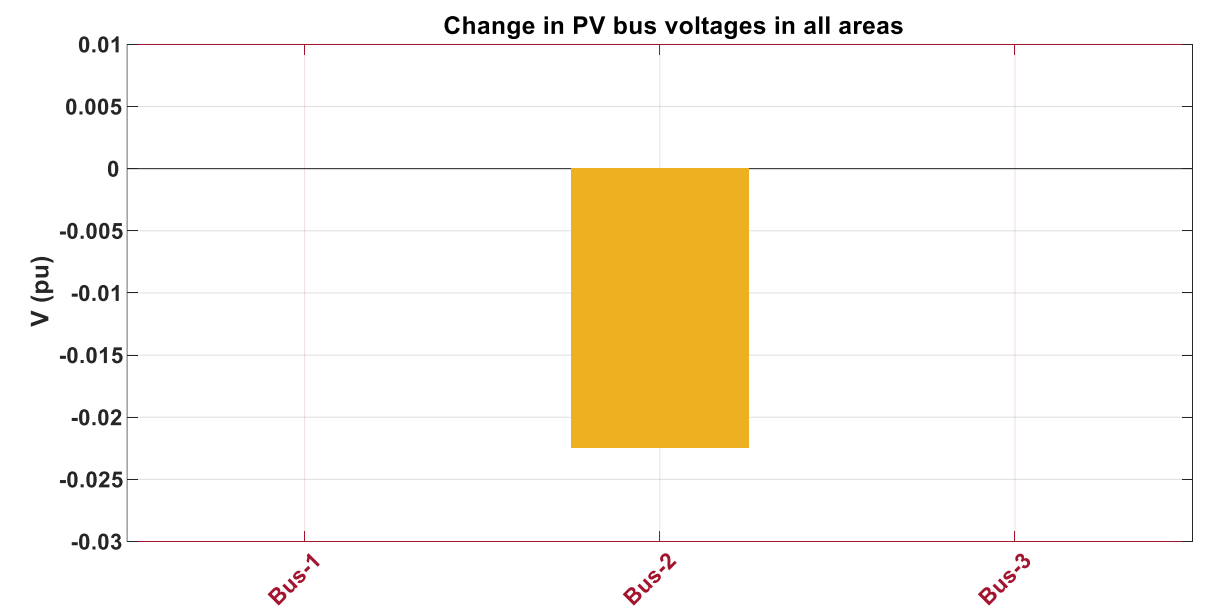

Fig. 4.16. Change in PV-Bus Voltages after Control (9-Bus Power System)

The voltage profile of the whole system before and after control is shown in Fig. 4.17 with the values given in Table 4.4. 


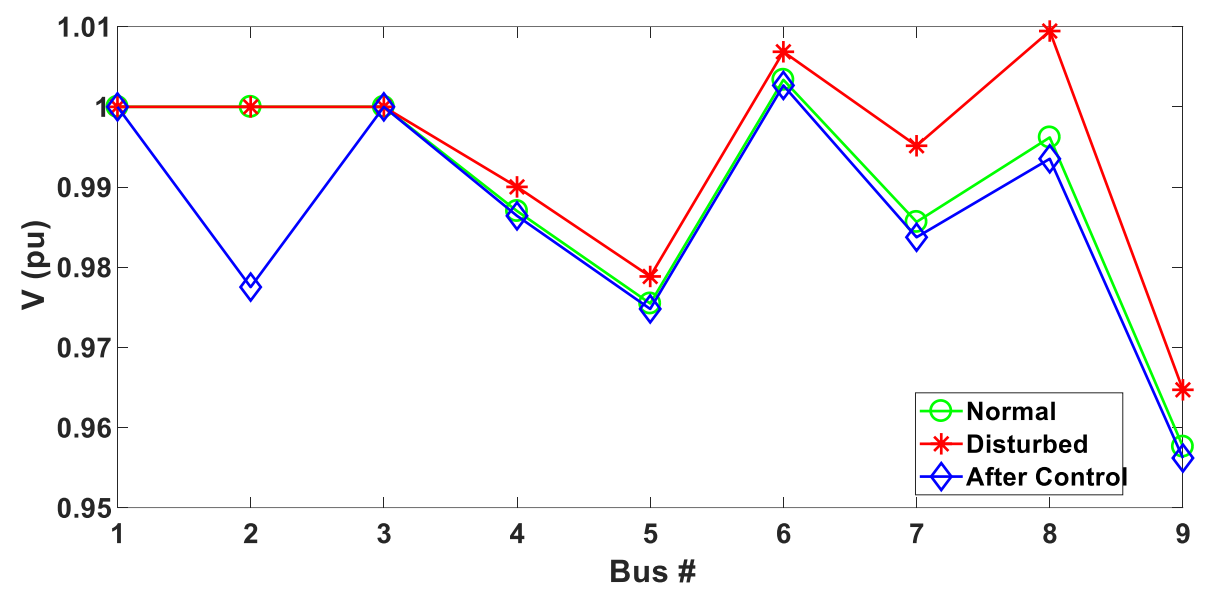

Fig. 4.17. Voltage Profile of the System (9-Bus Power System)

Table 4.4 Bus Voltages in Different Conditions (9-Bus Power System)

\begin{tabular}{|c|c|c|c|}
\hline \multirow{2}{*}{ Bus \# } & \multicolumn{3}{|c|}{ Voltage (pu) } \\
\cline { 2 - 4 } & Normal & $\begin{array}{c}\text { Disturbed } \\
\text { Before Control }\end{array}$ & $\begin{array}{c}\text { Disturbed } \\
\text { After Control }\end{array}$ \\
\hline $\mathbf{1}$ & 1 & 1 & 1 \\
\hline $\mathbf{2}$ & 1 & 1 & 0.978 \\
\hline $\mathbf{3}$ & 1 & 1 & 1 \\
\hline 4 & $\mathbf{0 . 9 8 7}$ & $\mathbf{0 . 9 9 0}$ & $\mathbf{0 . 9 8 6}$ \\
\hline $\mathbf{5}$ & 0.975 & 0.979 & 0.975 \\
\hline 6 & 1 & 1 & 1 \\
\hline $\mathbf{7}$ & 0.985 & 0.995 & 0.984 \\
\hline $\mathbf{8}$ & $\mathbf{0 . 9 9 6}$ & $\mathbf{1 . 0 1}$ & $\mathbf{0 . 9 9 3}$ \\
\hline $\mathbf{9}$ & 0.957 & 0.965 & 0.956 \\
\hline
\end{tabular}

\section{Scenario 2 - Multiple Perturbations:}

Now we would add introduce multiple perturbations into the system and observe how voltages are controlled by SVC in each area. We added inductive disturbance of $+30 \mathrm{MVAR}$ on bus-5 and bus-9 each and capacitive disturbance of -50MVAR on bus-7 as shown in Fig. 4.18. 


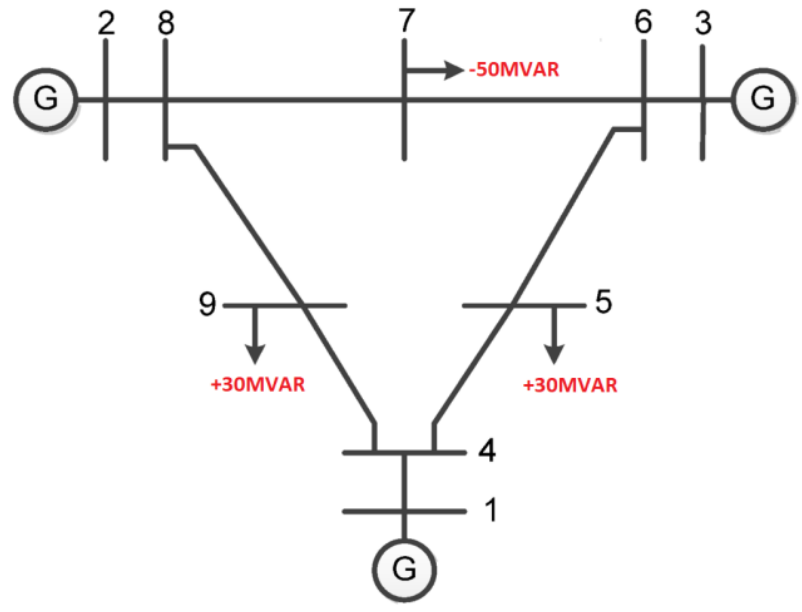

Fig. 4.18. Introducing Multiple Perturbations (9-Bus Power System)

All voltages before and after adding disturbances are shown in Fig. 4.19. Bus voltage changes in each VCA after disturbances are shown in Fig. 4.20.

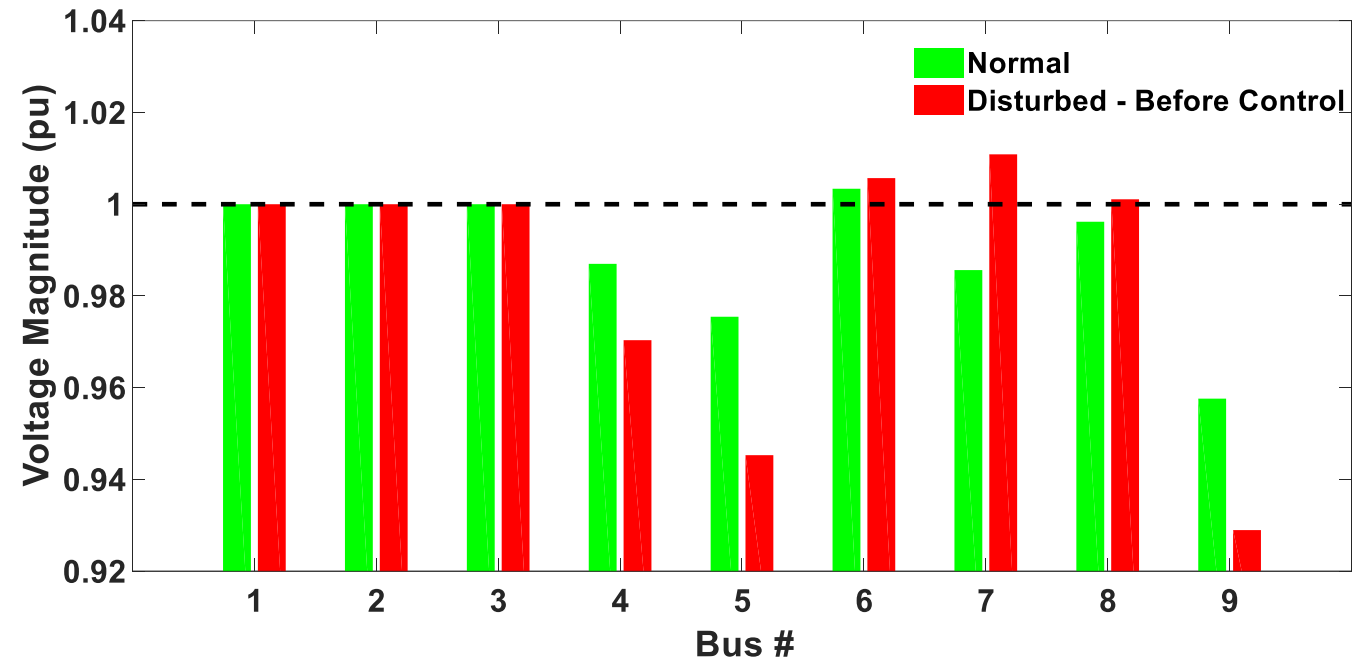

Fig. 4.19. Bus voltages before and after Perturbations (9-Bus Power System) 


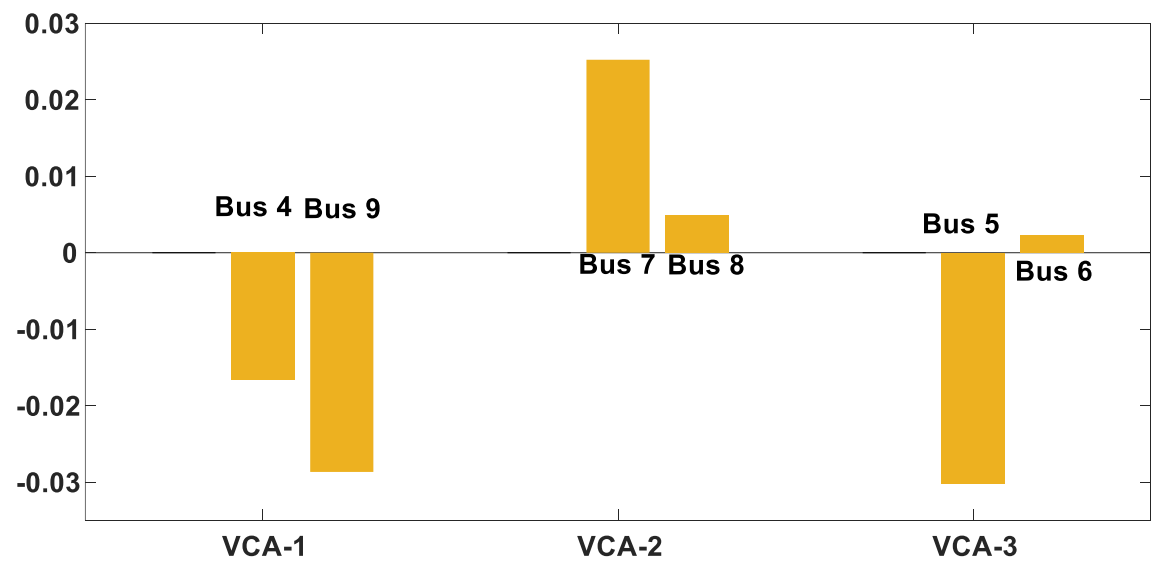

Fig. 4.20. Change in Bus voltages after Perturbations (9-Bus Power System)

After applying optimal voltage control, the pilot bus voltages were brought back at reference values as shown Fig. 4.21.
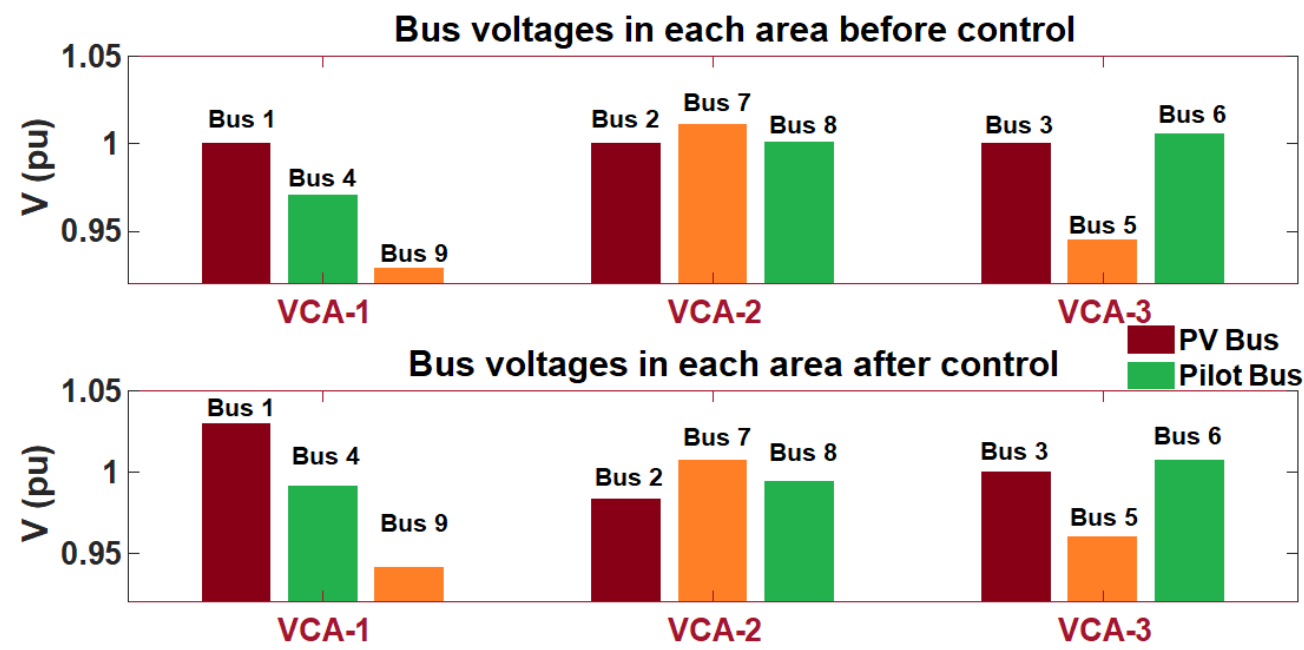

Fig. 4.21. Bus Voltages before and after Control (9-Bus Power System)

The change in generator bus voltages before and after control are shown in Fig. 4.22. The voltage profile of the whole system before and after control is shown in Fig. 4.23 with the values given in Table 4.5. 


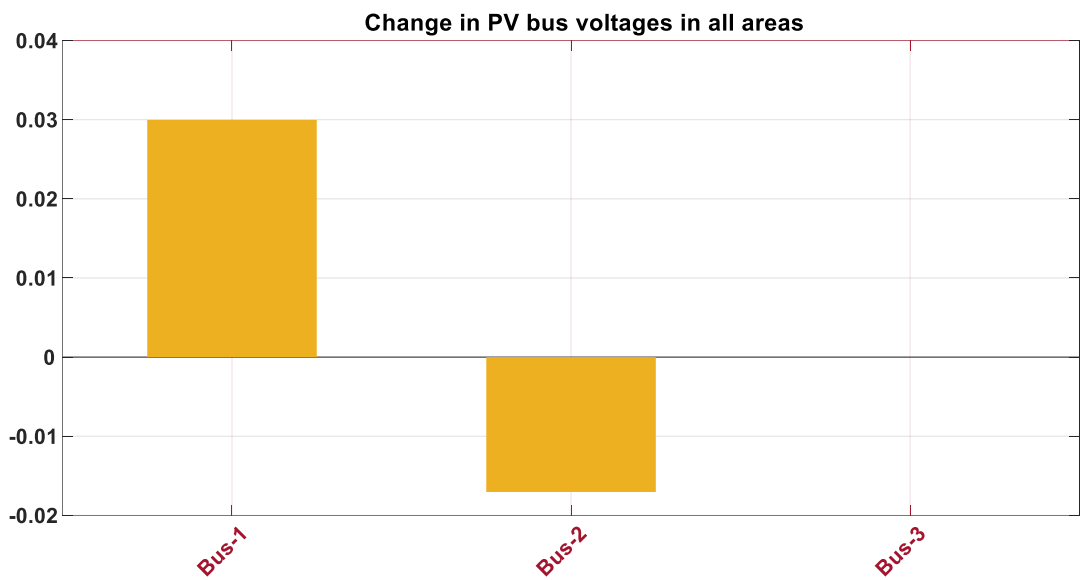

Fig. 4.22. Change in PV-Bus Voltages after Control (9-Bus Power System)

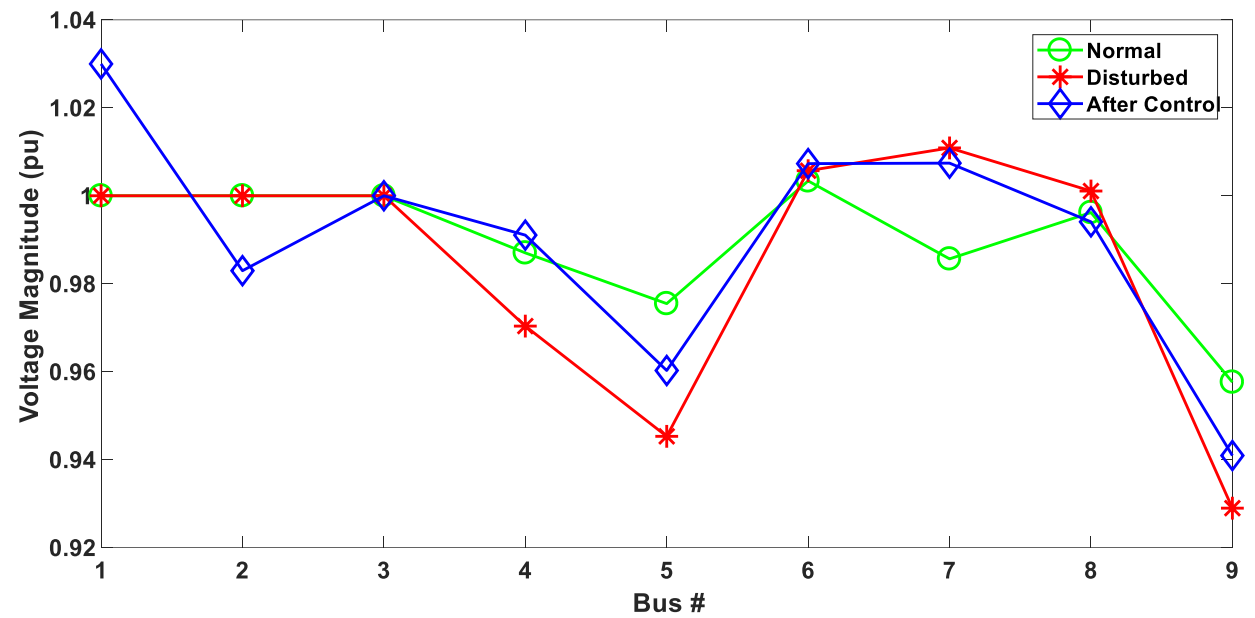

Fig. 4.23. Voltage Profile of the System (9-Bus Power System)

Table 4.5 Bus Voltages in Different Conditions (9-Bus Power System)

\begin{tabular}{|c|c|c|c|}
\hline \multirow{2}{*}{ Bus \# } & \multicolumn{3}{|c|}{ Voltage (pu) } \\
\cline { 2 - 4 } & Normal & $\begin{array}{c}\text { Disturbed } \\
\text { Before Control }\end{array}$ & $\begin{array}{c}\text { Disturbed } \\
\text { After Control }\end{array}$ \\
\hline $\mathbf{1}$ & 1 & 1 & 1.03 \\
\hline $\mathbf{2}$ & 1 & 1 & 0.983 \\
\hline $\mathbf{3}$ & 1 & 1 & 1 \\
\hline 4 & $\mathbf{0 . 9 8 7}$ & $\mathbf{0 . 9 7 0}$ & $\mathbf{0 . 9 9 1}$ \\
\hline $\mathbf{5}$ & 0.975 & 0.945 & 0.960 \\
\hline 6 & 1 & 1.006 & 1.007 \\
\hline $\mathbf{7}$ & 0.985 & 1.011 & 1.007 \\
\hline $\mathbf{8}$ & $\mathbf{0 . 9 9 6}$ & $\mathbf{1 . 0 0 1}$ & $\mathbf{0 . 9 9 4}$ \\
\hline $\mathbf{9}$ & 0.957 & 0.929 & 0.941 \\
\hline
\end{tabular}




\section{CHAPTER 4. OPTIMAL VOLTAGE CONTROL USING SVD}

In this system, each VCA had only one generator bus and we did not need to use SVD to find the optimal contribution of each generator to control pilot bus voltages. So, we studied another power system (39-bus system) to observe the use of SVD for optimal voltage control which gives optimal contribution of each PV-bus in an area (having more than one generators) so that the output change (i.e. change in pilot bus voltage) is maximized.

\subsubsection{9-Bus Power Network}

This system has ten (bus 30-39) voltage controlled buses (including slack bus). The active power demand is $6254.2 \mathrm{MW}$ while reactive power demand is 1274.9 MVAR. The voltages under normal operating conditions are given in Table 4.6.

Table 4.6 Voltages under Normal Operating Conditions (39-Bus Power System)

\begin{tabular}{|c|c|c|c|c|c|}
\hline Bus & $\mathbf{V}(\mathbf{p u})$ & Bus & $\mathbf{V}(\mathbf{p u})$ & Bus & $\mathbf{V}(\mathbf{p u})$ \\
\hline $\mathbf{1}$ & 1.039 & $\mathbf{1 4}$ & 1.012 & $\mathbf{2 7}$ & 1.038 \\
\hline $\mathbf{2}$ & 1.048 & $\mathbf{1 5}$ & 1.016 & $\mathbf{2 8}$ & 1.050 \\
\hline $\mathbf{3}$ & 1.031 & $\mathbf{1 6}$ & 1.033 & $\mathbf{2 9}$ & 1.050 \\
\hline $\mathbf{4}$ & 1.004 & $\mathbf{1 7}$ & 1.034 & $\mathbf{3 0}$ & 1.050 \\
\hline $\mathbf{5}$ & 1.006 & $\mathbf{1 8}$ & 1.032 & $\mathbf{3 1}$ & 0.982 \\
\hline $\mathbf{6}$ & 1.008 & 19 & 1.050 & $\mathbf{3 2}$ & 0.984 \\
\hline $\mathbf{7}$ & 0.998 & $\mathbf{2 0}$ & 0.991 & $\mathbf{3 3}$ & 0.997 \\
\hline $\mathbf{8}$ & 0.998 & $\mathbf{2 1}$ & 1.032 & $\mathbf{3 4}$ & 1.012 \\
\hline $\mathbf{9}$ & 1.038 & 22 & 1.050 & $\mathbf{3 5}$ & 1.049 \\
\hline $\mathbf{1 0}$ & 1.018 & $\mathbf{2 3}$ & 1.045 & $\mathbf{3 6}$ & 1.064 \\
\hline $\mathbf{1 1}$ & 1.013 & $\mathbf{2 4}$ & 1.038 & $\mathbf{3 7}$ & 1.028 \\
\hline $\mathbf{1 2}$ & 1.001 & $\mathbf{2 5}$ & 1.058 & $\mathbf{3 8}$ & 1.027 \\
\hline $\mathbf{1 3}$ & 1.015 & 26 & 1.053 & $\mathbf{3 9}$ & 1.030 \\
\hline
\end{tabular}

The voltage control areas and pilot points for this system obtained in previous chapter are shown in Fig. 4.24. The system was decomposed into six voltage control areas. 


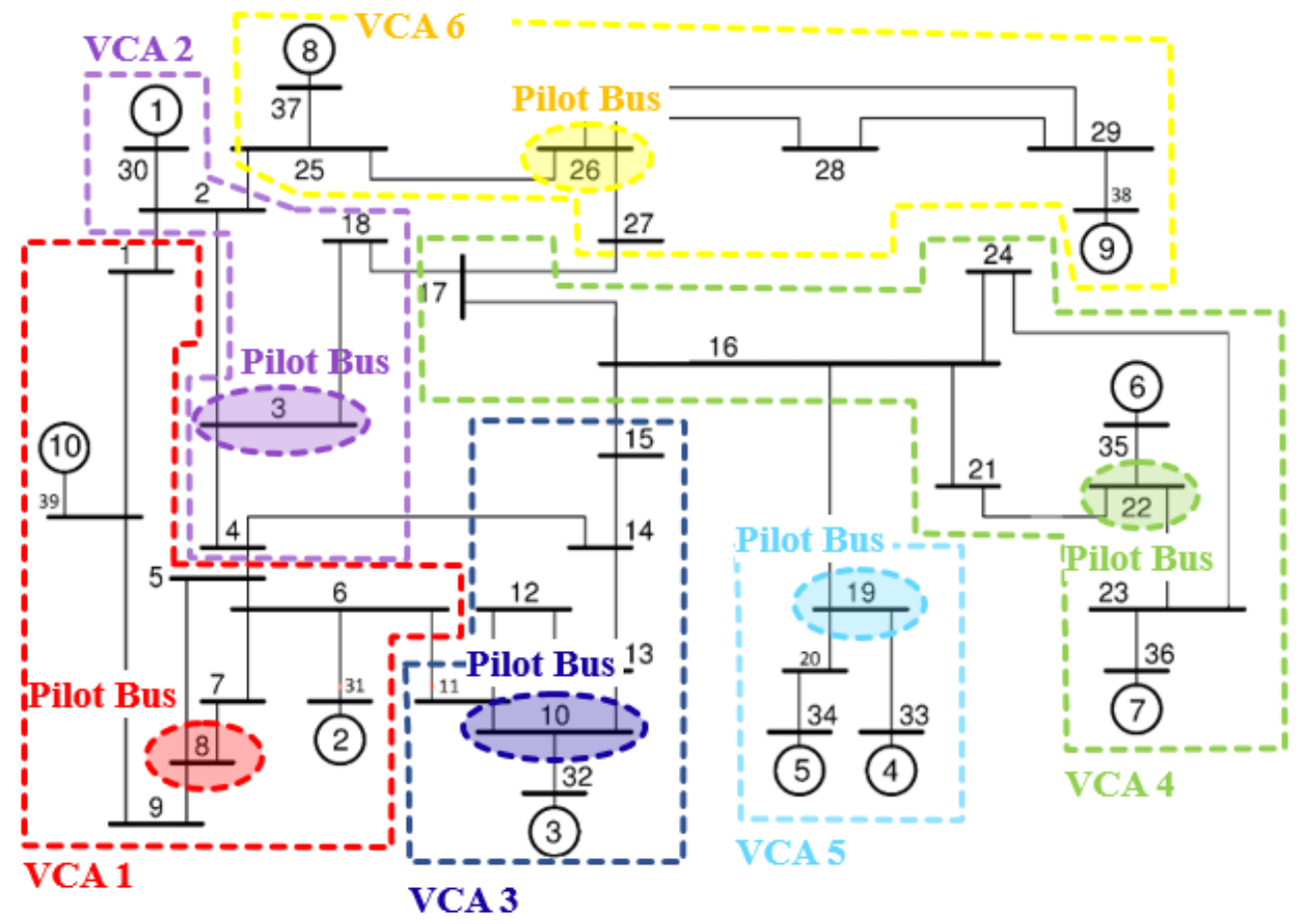

Fig. 4.24. Obtained VCAs and Pilot Buses (39-Bus Power System)

\subsubsection{Subjecting to Perturbation}

The data for each VCA is given in Table 4.7.

\section{Scenario 1 - Single Perturbation:}

Initially, we added single disturbances in different VCAs and observe the operation of SVC. We added a disturbance of -200MVAR in VCA4 on bus 23. The bus voltages before and after disturbance are shown in Fig. 4.25. 
CHAPTER 4. OPTIMAL VOLTAGE CONTROL USING SVD

Table 4.7 Data for each Voltage Control Area (39-Bus Power System)

\begin{tabular}{|c|c|c|c|c|c|c|}
\hline on & 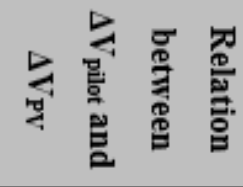 & 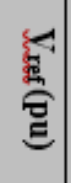 & 管 & 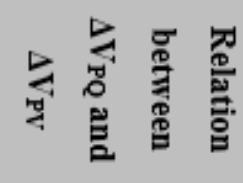 & 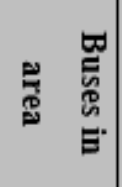 & 营 \\
\hline 웅 & 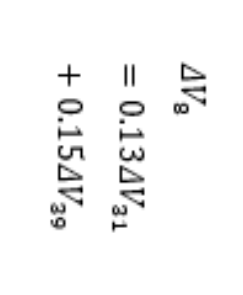 & $\begin{array}{l}0 \\
: \\
\vdots \\
\infty\end{array}$ & $\infty$ & 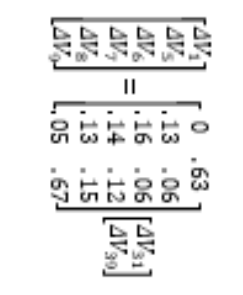 & 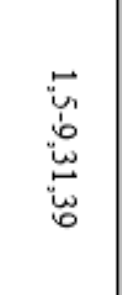 & $\widehat{\Omega}$ \\
\hline$\stackrel{\circ}{\circ}$ & 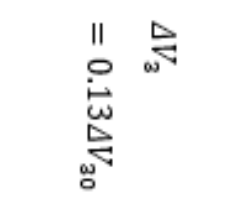 & $\overrightarrow{\mathrm{o}}$ & $\omega$ & 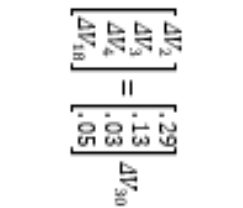 & $\begin{array}{l}\tilde{n} \\
\underline{w} \\
w \\
w \\
0\end{array}$ & 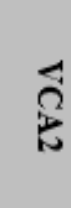 \\
\hline : & 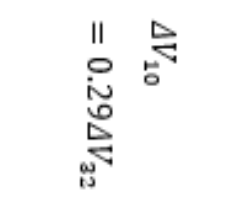 & $\vec{\circ}$ & 하 & 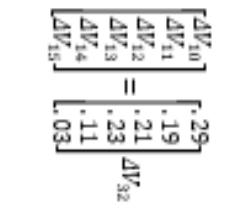 & 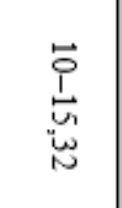 & 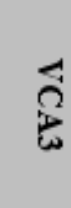 \\
\hline : & 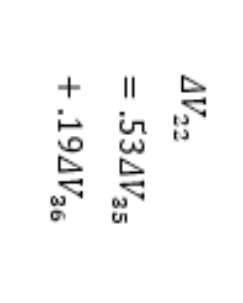 & 宫 & $N$ & 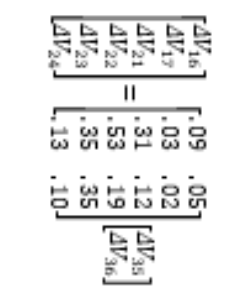 & 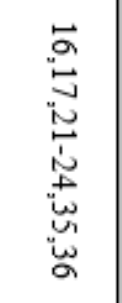 & $\overrightarrow{\tilde{R}}$ \\
\hline 응 & 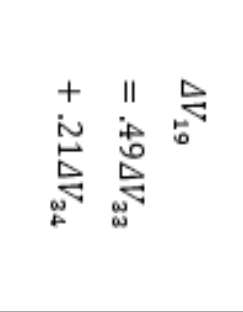 & $\overrightarrow{\dot{b}_{1}}$ & $\overrightarrow{0}$ & 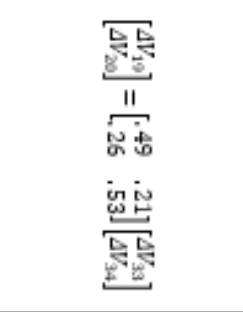 & 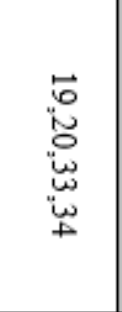 & 葛 \\
\hline 응 & 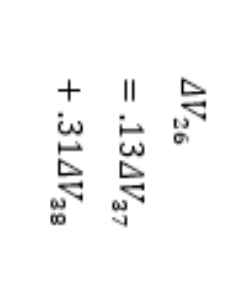 & $\overrightarrow{\dot{c}_{n}}$ & $\widetilde{\alpha}$ & 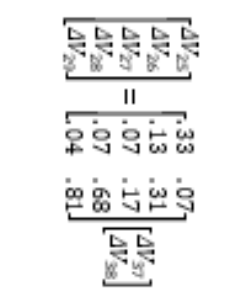 & 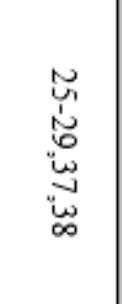 & 约 \\
\hline
\end{tabular}




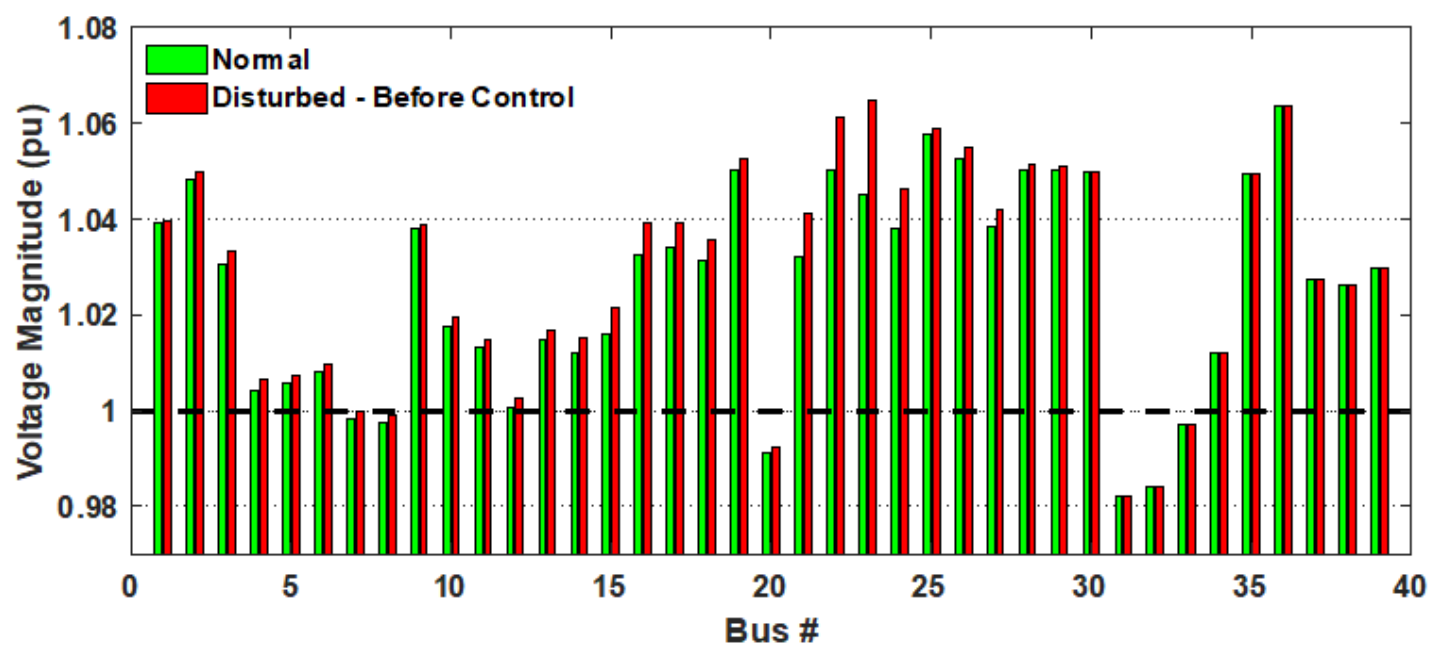

Fig. 4.25. Bus voltages before and after Perturbation in VCA4 on bus 23

$$
\text { (39-Bus Power System) }
$$

The change in bus voltages in all VCA after disturbance on bus 23 is shown in Fig. 4.26. It can be seen from the figure that the buses in in VCA4 are most sensitive to Q-injection in VCA4 compared to buses in other areas.

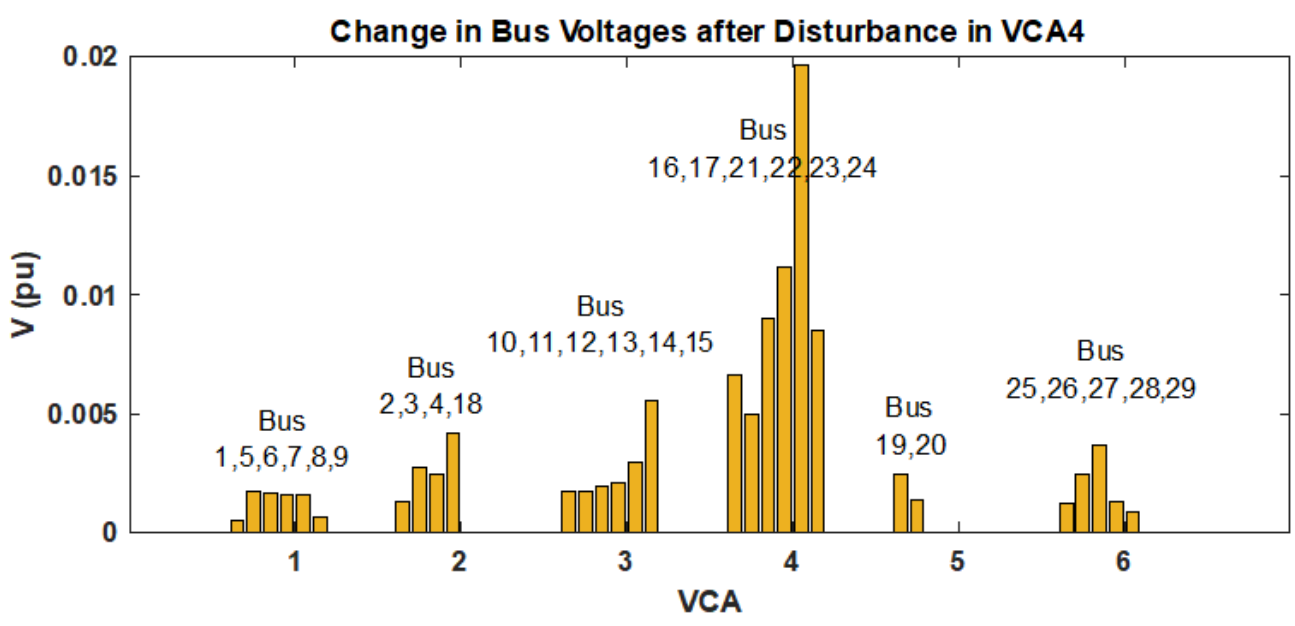

Fig. 4.26. Change in Bus voltages after Perturbation (39-Bus Power System)

The bus voltages before and after controlling pilot buses are shown in Fig. 4.27. While change in PV-bus voltages after control is shown in Fig. 4.28 and we can see again that only generators in VCA4 (i.e. Bus 35,36) are responding to this disturbance which is indicating decentralized operation of generators and it's also showing that intra-community $V-Q$ sensitivity is stronger than inter-community sensitivity. 
CHAPTER 4. OPTIMAL VOLTAGE CONTROL USING SVD
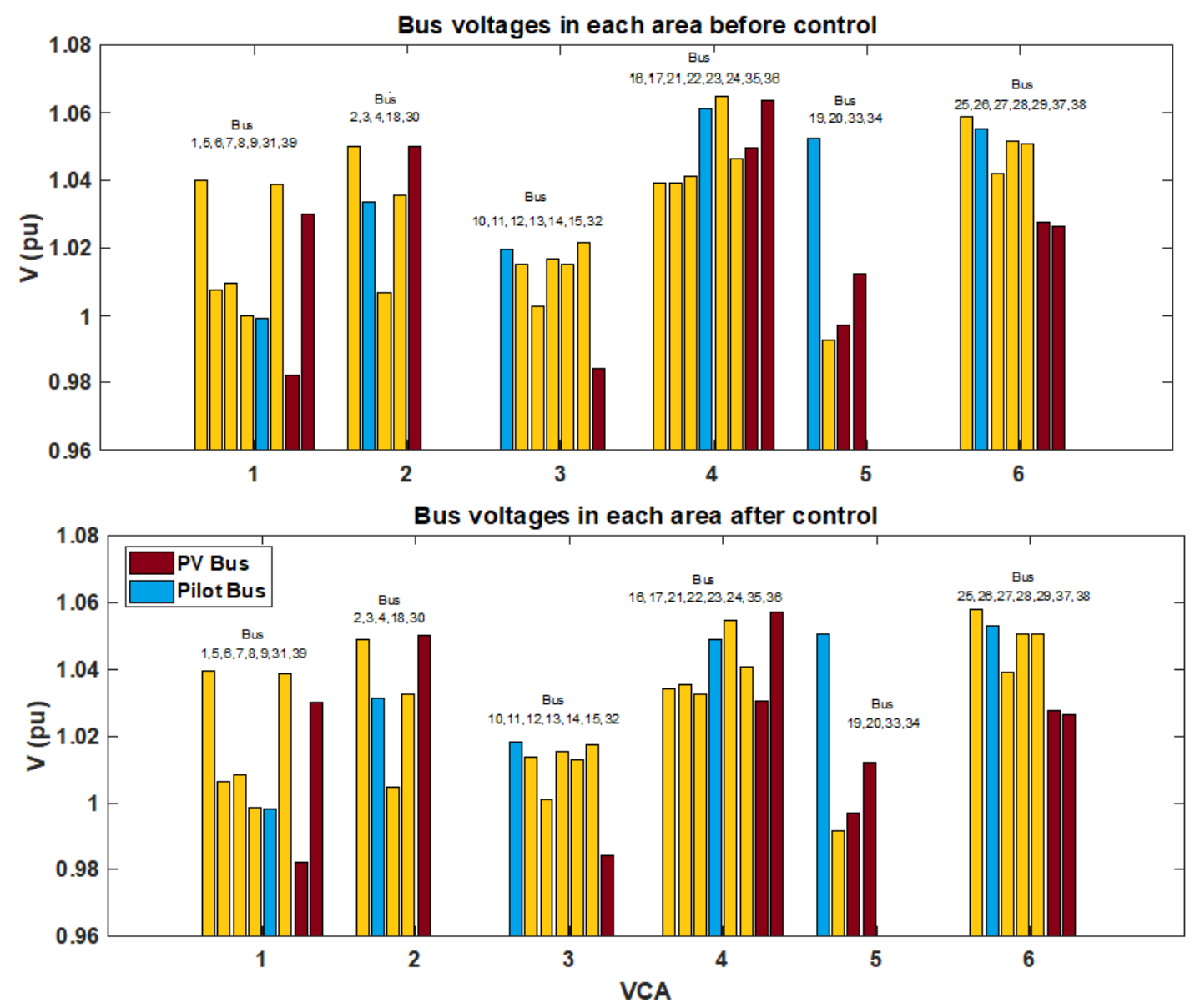

Fig. 4.27. Bus Voltages before and after Control (39-Bus Power System)

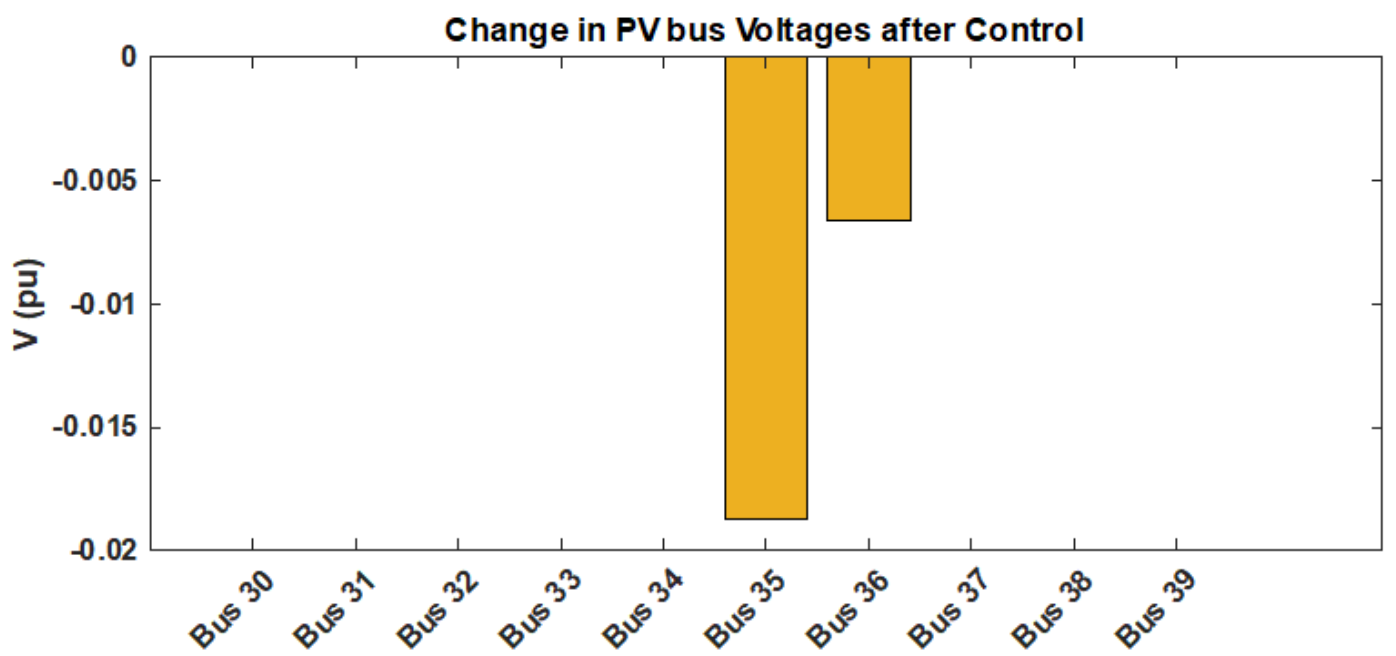

Fig. 4.28. Change in PV-Bus Voltages after Control (39-Bus Power System) 
VCA4 has two generator buses (Bus 35,36) and we know that its pilot bus (Bus 22) is more sensitive to Bus 35 compared to Bus 36. We can see in Fig. 28 that SVC of this area is changing voltage reference of Bus-35 more than Bus-36. So, sensitivity is inherent in this voltage control which is based on singular value decomposition (SVD).

\section{Scenario 2 - Multiple Perturbations:}

In this case, we added multiple disturbances into system (-150MVAR on bus 9 in VCA1, $+100 \mathrm{MVAR}$ on bus 18 in VCA2 and +200MVAR on bus 25 in VCA6). The voltages of buses before and after disturbances are shown in Fig. 4.29.

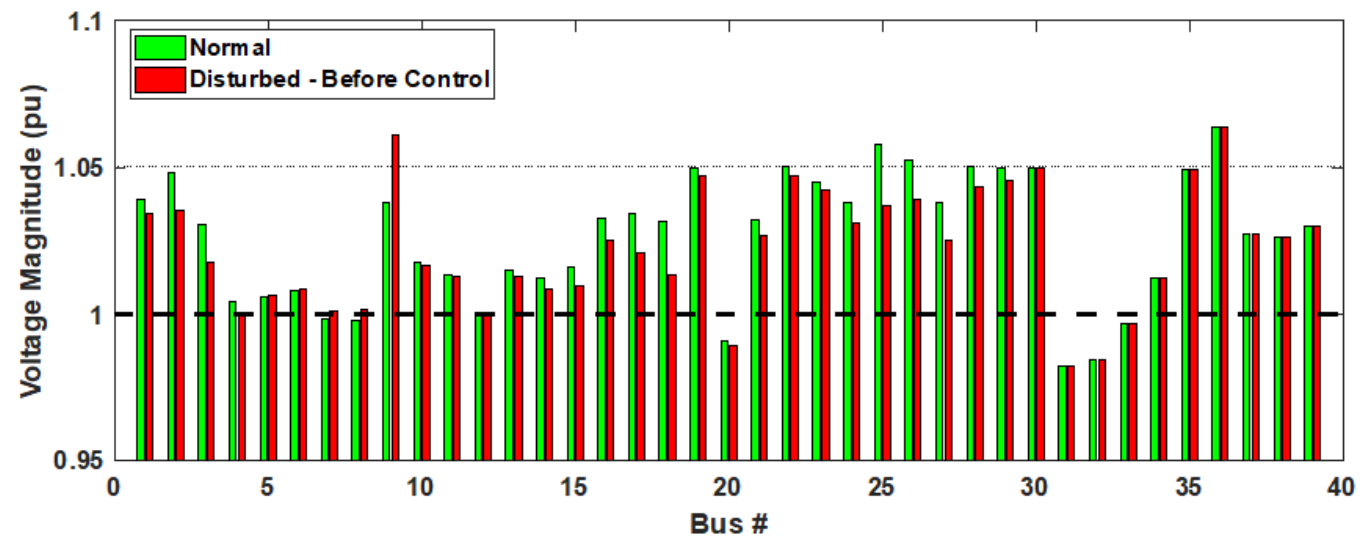

Fig. 4.29. Bus voltages before and after Perturbations (39-Bus Power System)

The changes in pilot bus voltages in all VCAs after disturbances are shown in Fig. 4.30. It can be seen from the figure that the pilot buses in VCA1,2 \& 6 have most variations because disturbances were added in these areas which indicates that intra-community sensitivity is higher compared to inter-community sensitivity. 


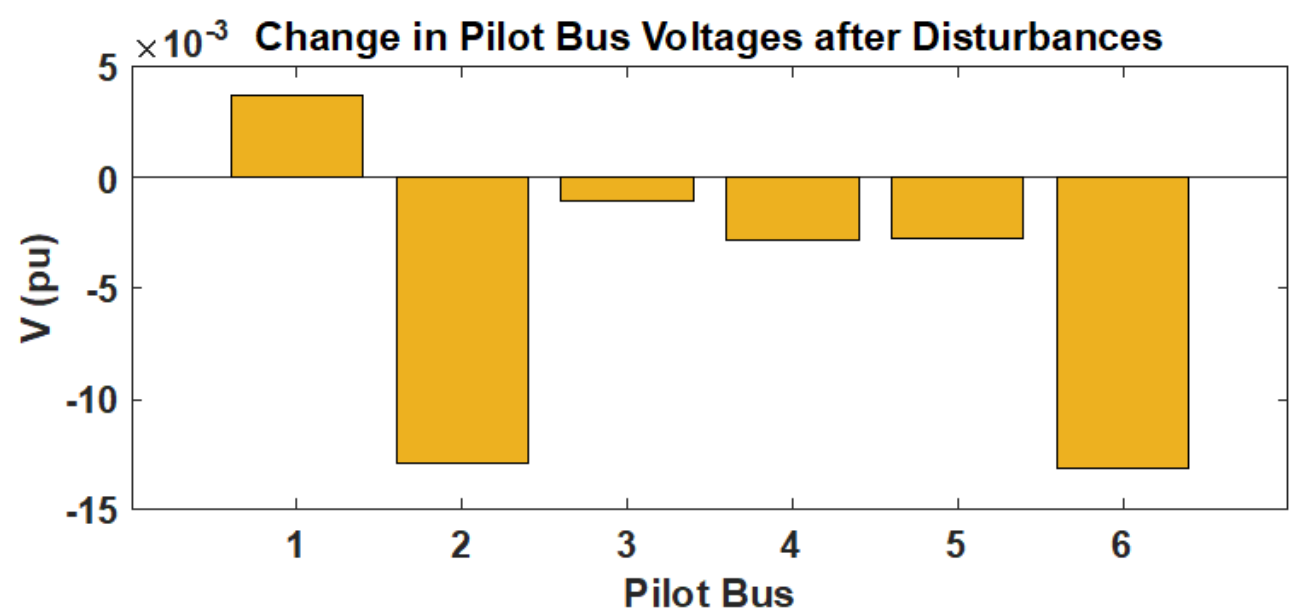

Fig. 4.30. Change in pilot bus voltages after perturbations (39-Bus Power System)

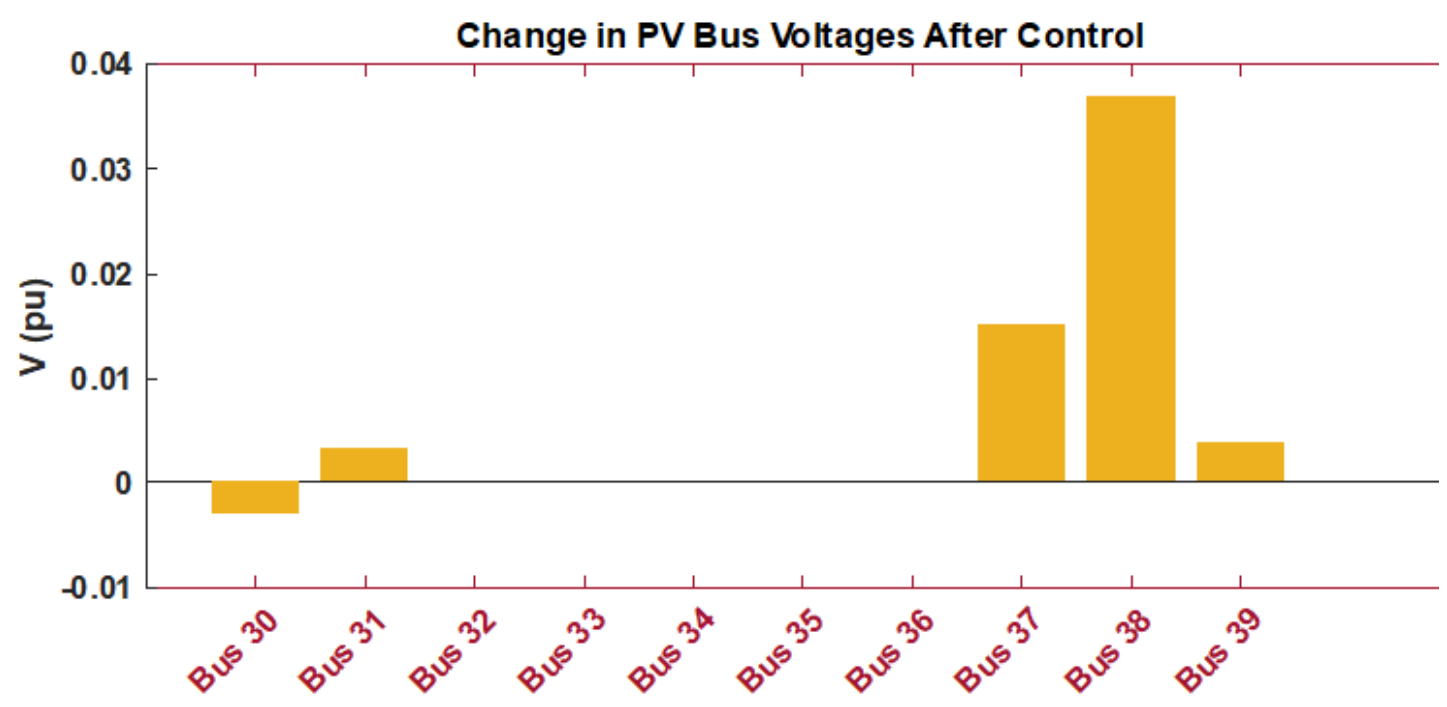

Fig. 4.31. Change in PV bus voltages after control (39-Bus Power System)

The PV bus voltages after controlling the pilot bus voltages are shown in Fig. 31. It can be seen from the figure that only generators of areas VCA1, VCA2 and VCA4 and responding to these disturbances which again proves decentralized operation of secondary voltage controllers. The voltage profile of the whole system before and after control is shown in Fig. 4.32 with the values given in Table 4.8. 


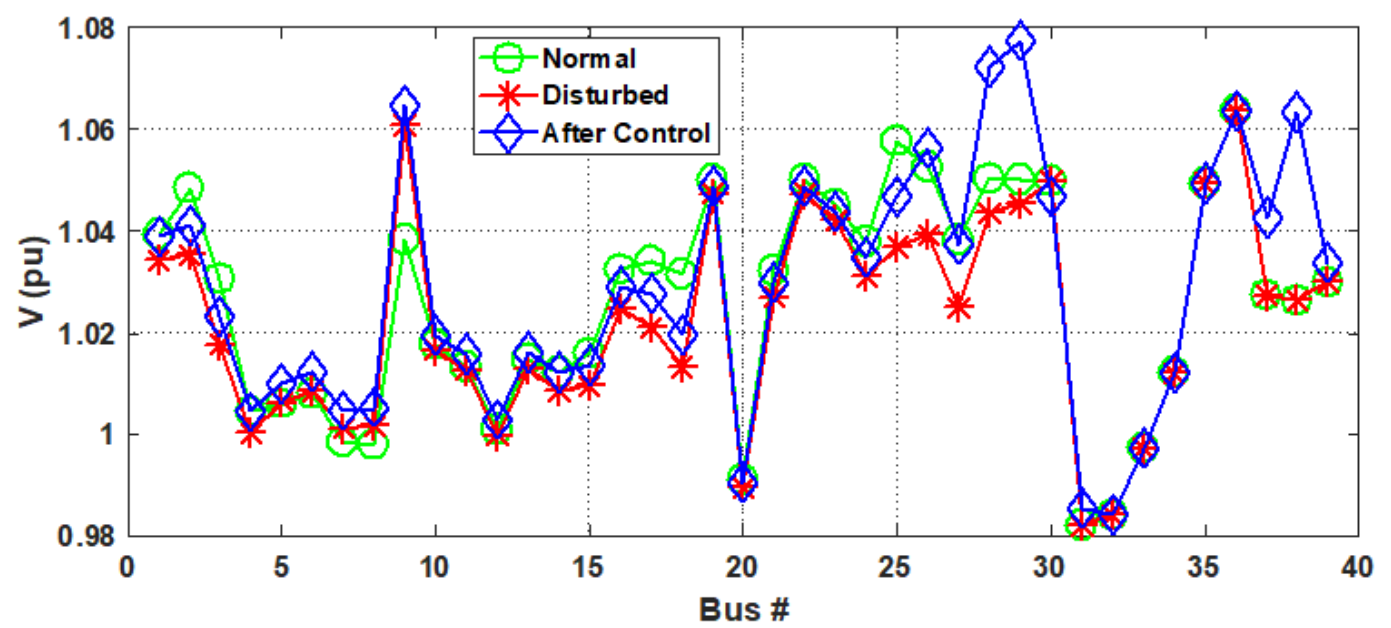

Fig. 4.32. Voltage Profile of the System (39-Bus Power System)

Table 4.8 Bus Voltages in Different Conditions (39-Bus Power System)

\begin{tabular}{|c|c|c|c|c|c|c|c|c|}
\hline \multirow[b]{2}{*}{ Bus } & \multicolumn{2}{|c|}{$V(p u)$} & \multirow[b]{2}{*}{ Bus } & \multicolumn{2}{|c|}{$V(p u)$} & \multirow[b]{2}{*}{ Bus } & \multicolumn{2}{|c|}{$V(p u)$} \\
\hline & Normal & $\begin{array}{c}\text { After } \\
\text { Control }\end{array}$ & & Normal & $\begin{array}{c}\text { After } \\
\text { Control }\end{array}$ & & Normal & $\begin{array}{c}\text { After } \\
\text { Control }\end{array}$ \\
\hline 1 & 1.039 & 1.039 & 14 & 1.012 & 1.012 & 27 & 1.038 & 1.037 \\
\hline 2 & 1.048 & 1.041 & 15 & 1.016 & 1.014 & 28 & 1.050 & 1.072 \\
\hline 3 & 1.031 & 1.023 & 16 & 1.033 & 1.029 & 29 & 1.050 & 1.077 \\
\hline 4 & 1.004 & 1.004 & 17 & 1.034 & 1.028 & 30 & 1.050 & 1.047 \\
\hline 5 & 1.006 & 1.010 & 18 & 1.032 & 1.020 & 31 & 0.982 & 0.985 \\
\hline 6 & 1.008 & 1.012 & 19 & 1.050 & 1.049 & 32 & 0.984 & 0.984 \\
\hline 7 & 0.998 & 1.005 & 20 & 0.991 & 0.990 & 33 & 0.997 & 0.997 \\
\hline 8 & 0.998 & 1.005 & 21 & 1.032 & 1.030 & 34 & 1.012 & 1.012 \\
\hline 9 & 1.038 & 1.065 & 22 & 1.050 & 1.049 & 35 & 1.049 & 1.049 \\
\hline 10 & 1.018 & 1.020 & 23 & 1.045 & 1.044 & 36 & 1.064 & 1.064 \\
\hline 11 & 1.013 & 1.016 & 24 & 1.038 & 1.035 & 37 & 1.028 & 1.043 \\
\hline 12 & 1.001 & 1.003 & 25 & 1.058 & 1.047 & 38 & 1.027 & 1.063 \\
\hline 13 & 1.015 & 1.016 & 26 & 1.053 & 1.056 & 39 & 1.030 & 1.034 \\
\hline
\end{tabular}

\subsubsection{Comparison between $S e n-V C$ and $O p t-V C$ :}

We compared the performance of optimal voltage control $(O p t-V C)$ presented here with sensitivity based voltage control $(S e n-V C)$ which uses sensitivities of pilot bus voltages on $P V$ bus voltages (i.e. changing only that $P V$ bus voltage to which pilot bus is 


\section{CHAPTER 4. OPTIMAL VOLTAGE CONTROL USING SVD}

most sensitive). We compared the energy function for change in input (i.e. $\Delta \boldsymbol{V}_{\boldsymbol{P V}}^{\boldsymbol{T}} \boldsymbol{\Delta} \boldsymbol{V}_{\boldsymbol{P V}}$ ) for same change in pilot bus voltage $\left(\boldsymbol{\Delta} \boldsymbol{V}_{\text {pilot }}\right)$ for the case (i.e. single disturbance on bus 23$)$ shown in Fig. 4.25. We define change in input energy function ( $\mathrm{L}_{2}$ norm of inputs) as in (4.18).

$$
\Delta \mathrm{E}_{\text {input }}=\Delta V_{P V}^{T} \Delta V_{P V}
$$

We also define change in output energy function as

$$
\Delta \mathrm{E}_{\text {output }}=\Delta V_{\text {pilot }}^{2}
$$

Then we calculate the energy gain $\left(\mathbf{G}_{\boldsymbol{E}}\right)$ viz defined in (4.20)

$$
\mathrm{G}_{E}=\frac{\Delta \mathrm{E}_{\text {output }}}{\Delta \mathrm{E}_{\text {input }}}
$$

Gain in energy $\left(\mathrm{G}_{\mathrm{E}}\right)$ given in (4.20) gives us the proportion of input energy being put in for control of pilot bus voltage which is used in changing pilot bus voltage because we know that this input energy would also change voltages of buses other than pilot bus.

For the case being discussed here (i.e. single disturbance on bus 23),

$$
\begin{gathered}
\Delta \mathrm{E}_{\text {output }}=\Delta V_{\text {pilot }}^{2}=(-.0123)^{2}=0.00015129 \\
\Delta \mathrm{E}_{\text {input }}(O p t-V C)=\Delta V_{35}^{2}+\Delta V_{36}^{2}=0.00039458 \\
\Delta \mathrm{E}_{\text {input }}(\text { Sen }-V C)=\Delta V_{35}^{2}=0.00044944 \\
\mathrm{G}_{E}(O p t-V C)=\frac{0.00015129}{0.00039458}=0.3834=38.3 \% \\
\mathrm{G}_{E}(\text { Sen }-V C)=\frac{0.00015129}{0.00044944}=0.3366=33.6 \%
\end{gathered}
$$


CHAPTER 4. OPTIMAL VOLTAGE CONTROL USING SVD

It's evident from the results that our approach (Opt-VC) is giving better performance compared to the approach based on sensitivity (i.e. Sen-VC) as shown graphically in Fig. 4.33 .

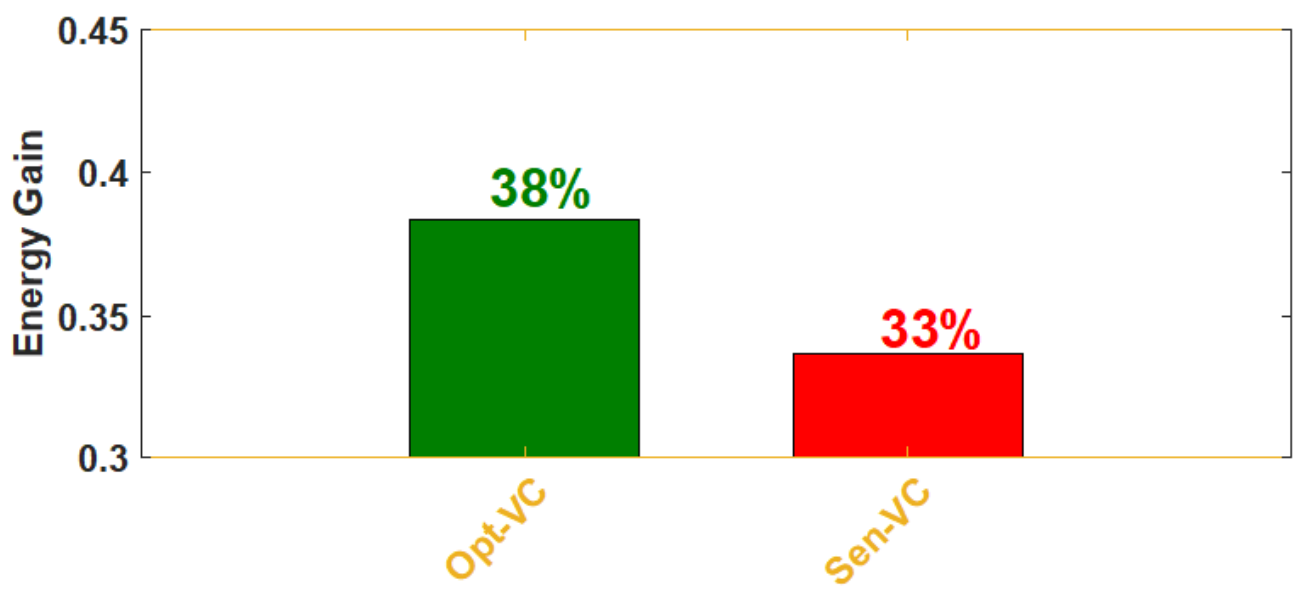

Fig. 4.33. Comparison of Energy Gain between Opt-VC and Sen-VC (39-Bus Power System) 


\section{Chapter 5}

\section{Conclusion and Future Work}

In this thesis, an optimal secondary voltage control (SVC) approach is presented. The presented voltage control technique has three steps:

1. Division of a power system into voltage control areas (VCAs)

2. Selection of pilot bus in each voltage control area

3. Control of pilot bus voltage

Initially, a large power system is decomposed into small voltage control areas. Each area has enough reactive power supply to balance its reactive power demand to be able to maintain its voltage profile within normal limits. A fast community detection algorithm was used for the identification of voltage control areas which detects dense and sparser areas in a network based on $V-Q$ sensitivity matrix. The advantage of the algorithm is that it not only divides a system into areas but also gives the optimal number of voltage control areas a network should be divided into, using a measure called modified modularity.

After identifying VCAs, a pilot bus is selected in each VCA as a representative of the voltage profile of its whole VCA. Electrical distance based approach was used to select pilot buses which is one of the most commonly used approaches for pilot points selection in literature. This approach selects pilot buses such that they give a good observability of the voltage profile of their whole area. 


\section{CHAPTER 5. CONCLUSION AND FUTURE WORK}

The final step in secondary voltage control (SVC) is to keep the voltages of these pilot buses constant before and after disturbances. Singular value decomposition of $V-Q$ sensitivity matrix was used to give a good controllability of the voltages of these pilot buses. The objective is to put in minimum input (i.e. change in generator bus voltages) to bring a required change in output (i.e. change in pilot bus voltage).

The presented approach is tested on two standard IEEE test power systems namely 9Bus and 39-Bus systems and satisfactory results were obtained. A computational time comparison is also performed between the fast community detection algorithm, presented in this thesis, and original-GN algorithm and it is shown that the algorithm presented requires much less time to detect VCAs especially for large power systems (i.e. $4 \%$ timereduction for 9 -Bus system and $73 \%$ time-reduction for 39-Bus system). Finally, through simulation results, it is shown that the presented optimal voltage control $(O p t-V C)$ approach gives a better approach compared to sensitivity based voltage control (Sen-VC) in terms of required input energy to put into the system to bring required change in output.

As discussed in chapter 2, there are different approaches in literature for network partitioning, pilot points selection and voltage control. Although, a comparison of the presented voltage control approach has been discussed in chapter 3 and chapter 4 but a detailed comparative study of the presented approach with existing secondary voltage approaches would be performed in future work of this research. Apart from this, there are many other factors which can be considered to improve the performance of the presented approach. The pilot points selection approach, for example, based on electrical distances, used in this thesis, gives a good observability of the voltage profile of the whole area but it performance in terms of controllability is not as effective as other approaches. The factors which are usually considered while selecting pilot buses are Observability, Controllability, Robustness and Sensitivity.

By considering these criteria, we believe that the performance of presented voltage control can be further improved if some advanced pilot bus selection approaches are used e.g. pilot points selection using modes of sensitivity of $V-Q$ sensitivity. These things are under consideration for future work of this research. 


\section{Appendix}

\section{Fast Decoupled Load Flow (FDLF) Jacobian:}

The relation between node current injections and node voltages in a power network is described as in (a).

$$
I_{\text {bus }}=\boldsymbol{Y}_{\text {bus }} \boldsymbol{V}_{\text {bus }}
$$

where $\mathbf{I}_{\text {bus }}$ is the vector of the injected bus currents and $\mathbf{V}_{\text {bus }}$ is the vector of the bus voltages measured w.r.t reference bus. Y bus is called the bus admittance matrix and its diagonal and non-diagonal elements are calculated as in (b) and (c) respectively, Saadat [6].

$$
\begin{aligned}
& Y_{i i}=\sum_{j=0}^{n} y_{i j} \quad j \neq i \\
& Y_{i j}=Y_{j i}=-y_{i j}
\end{aligned}
$$

$y_{i j}$ is the admittance connected between node $i$ and node $j$. The susceptance matrix $(\mathbf{B})$ is calculated from the admittance matrix as given in (d). Using this susceptance matrix (B), we can calculate B" as given by (3.3a) in chapter 3 .

$$
\boldsymbol{B}=\operatorname{imag}\left(\boldsymbol{Y}_{\text {bus }}\right)
$$

\section{MATLAB Code for Optimal Network Partitioning to detect voltage control areas:}

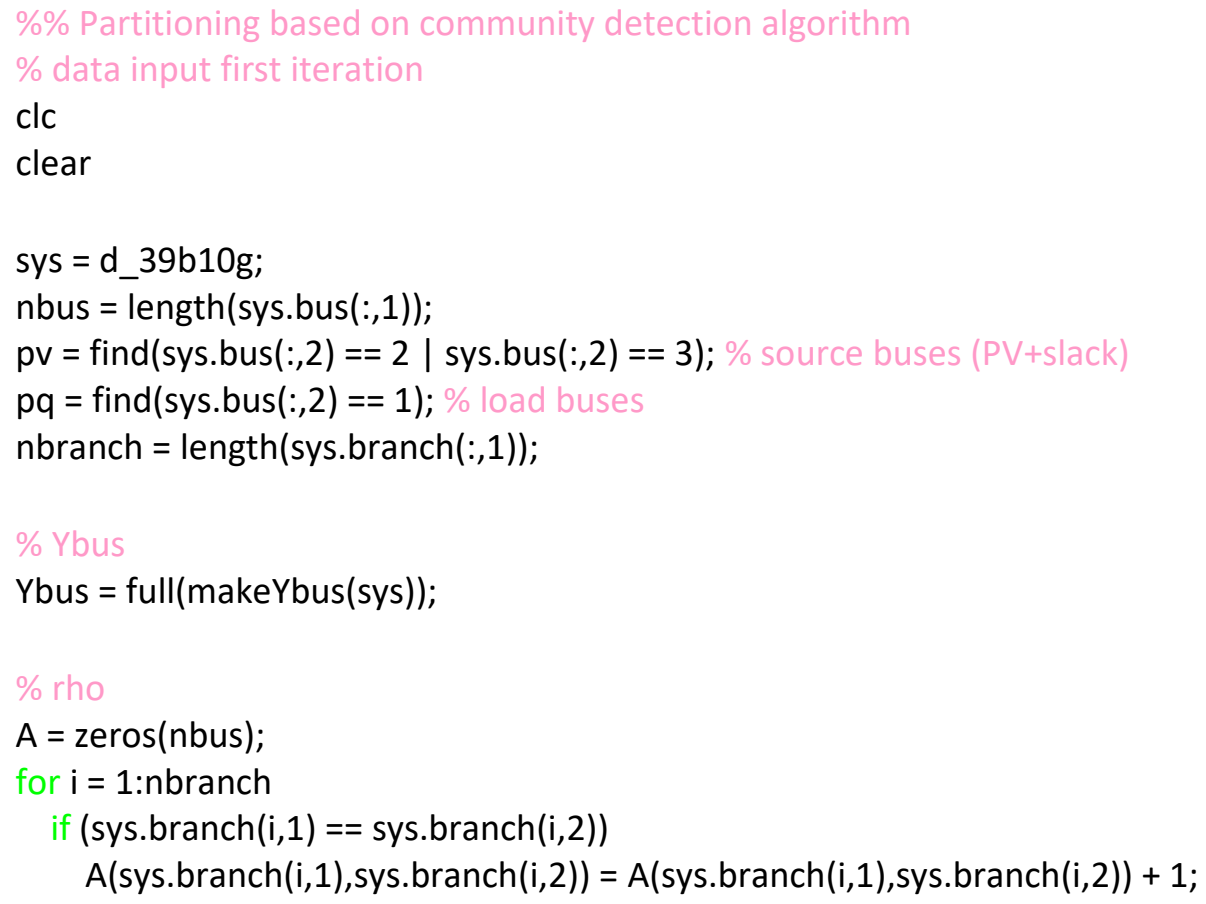




\section{APPENDIX. MATLAB PROGRAM FOR SVC}

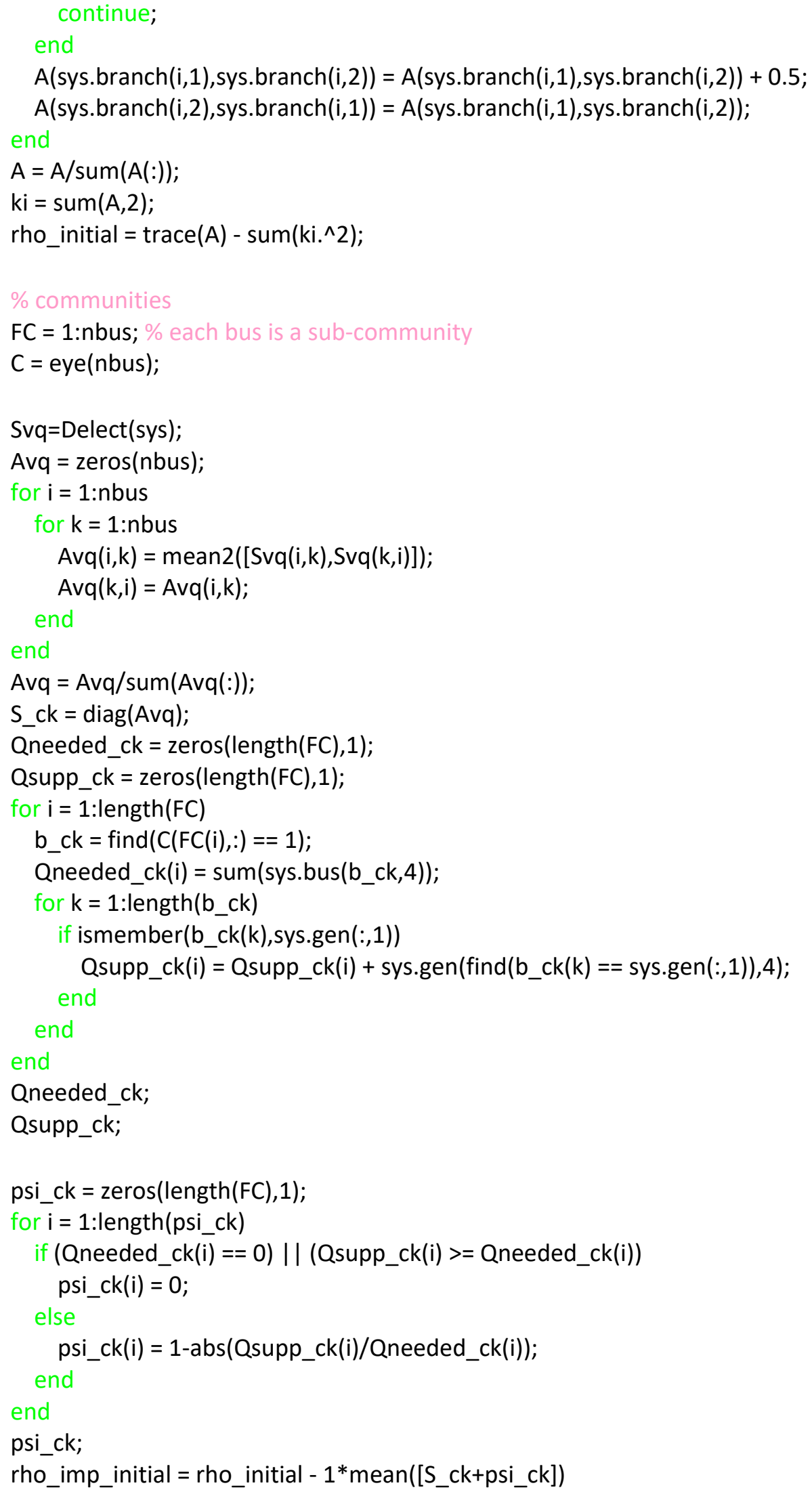




\section{APPENDIX. MATLAB PROGRAM FOR SVC}

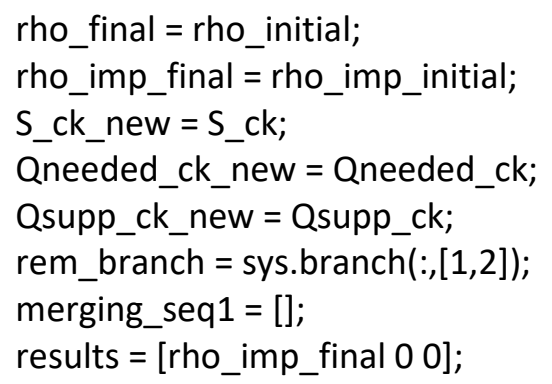

\section{$\% \%$ Start Merging}

\section{while (1)}

rho_new $=[]$;

rho_imp_new = [];

for $\mathrm{nbr}=1$ :nbranch

temp_C $=\mathrm{C}$;

temp_FC $=F C$;

temp_S_ck = S_ck_new;

temp_Qneeded_ck = Qneeded_ck_new;

temp_Qsupp_ck = Qsupp_ck_new;

temp_psi_ck = zeros(nbus,1);

temp_branch =rem_branch;

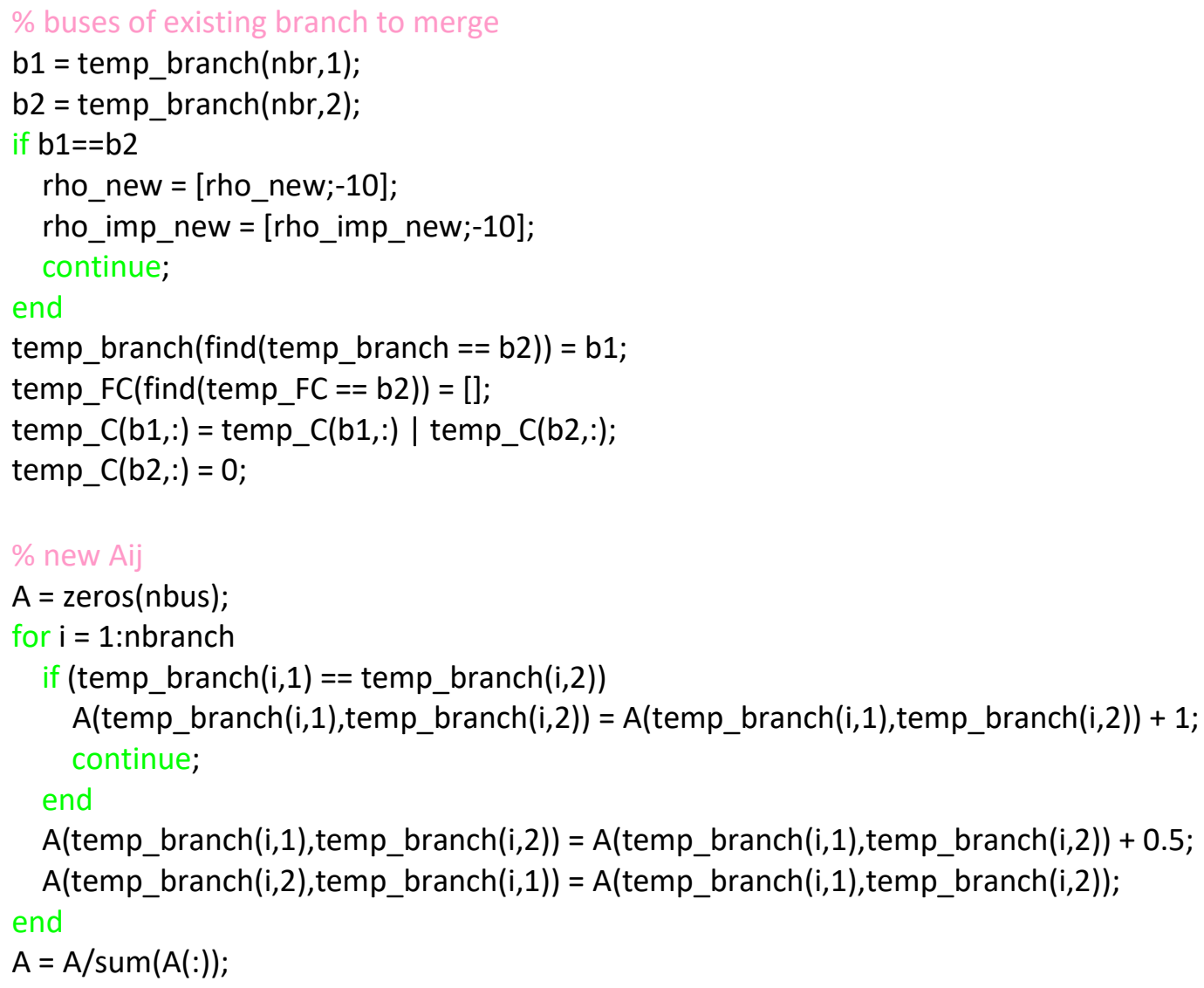




\section{APPENDIX. MATLAB PROGRAM FOR SVC}

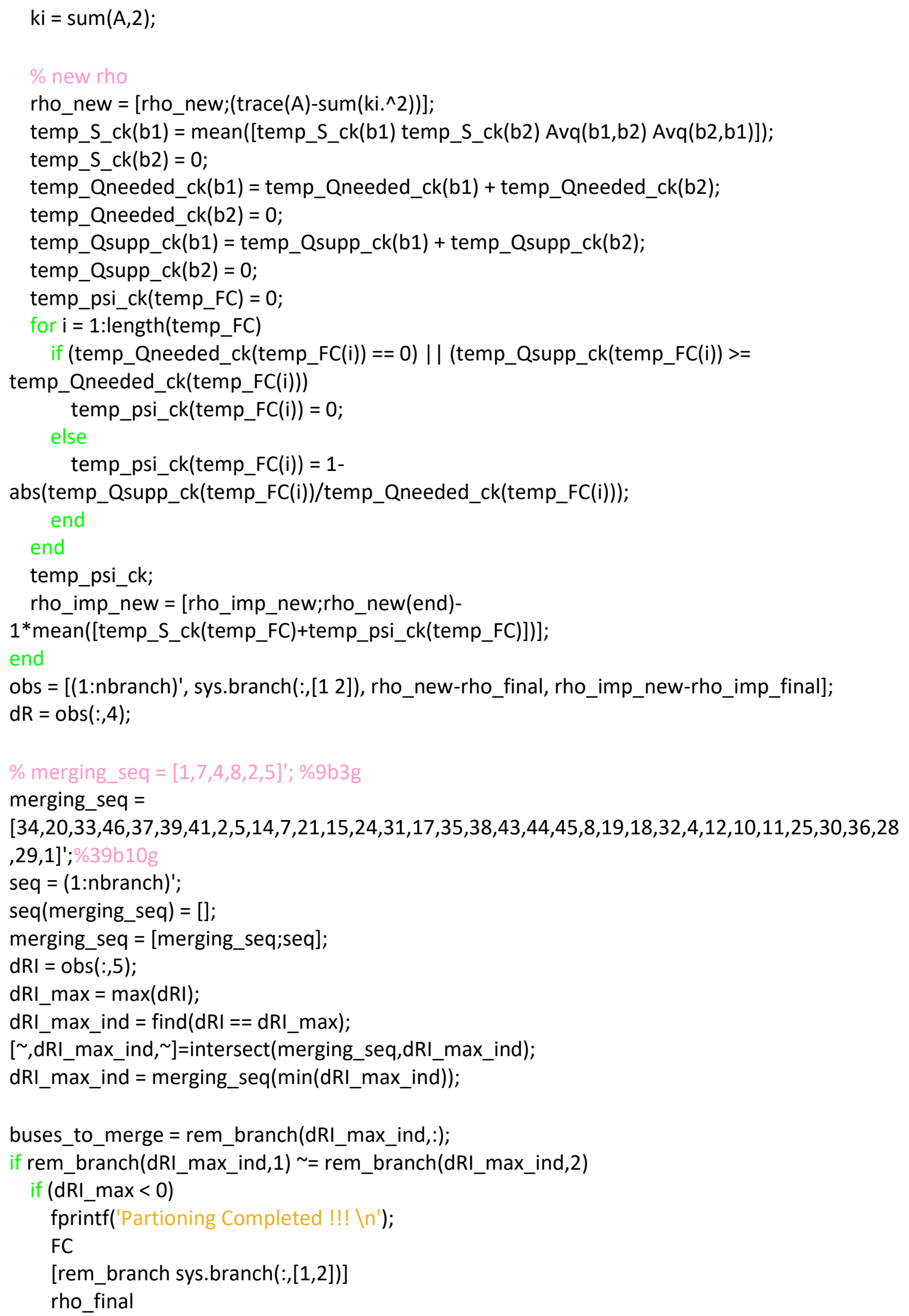




\section{APPENDIX. MATLAB PROGRAM FOR SVC}

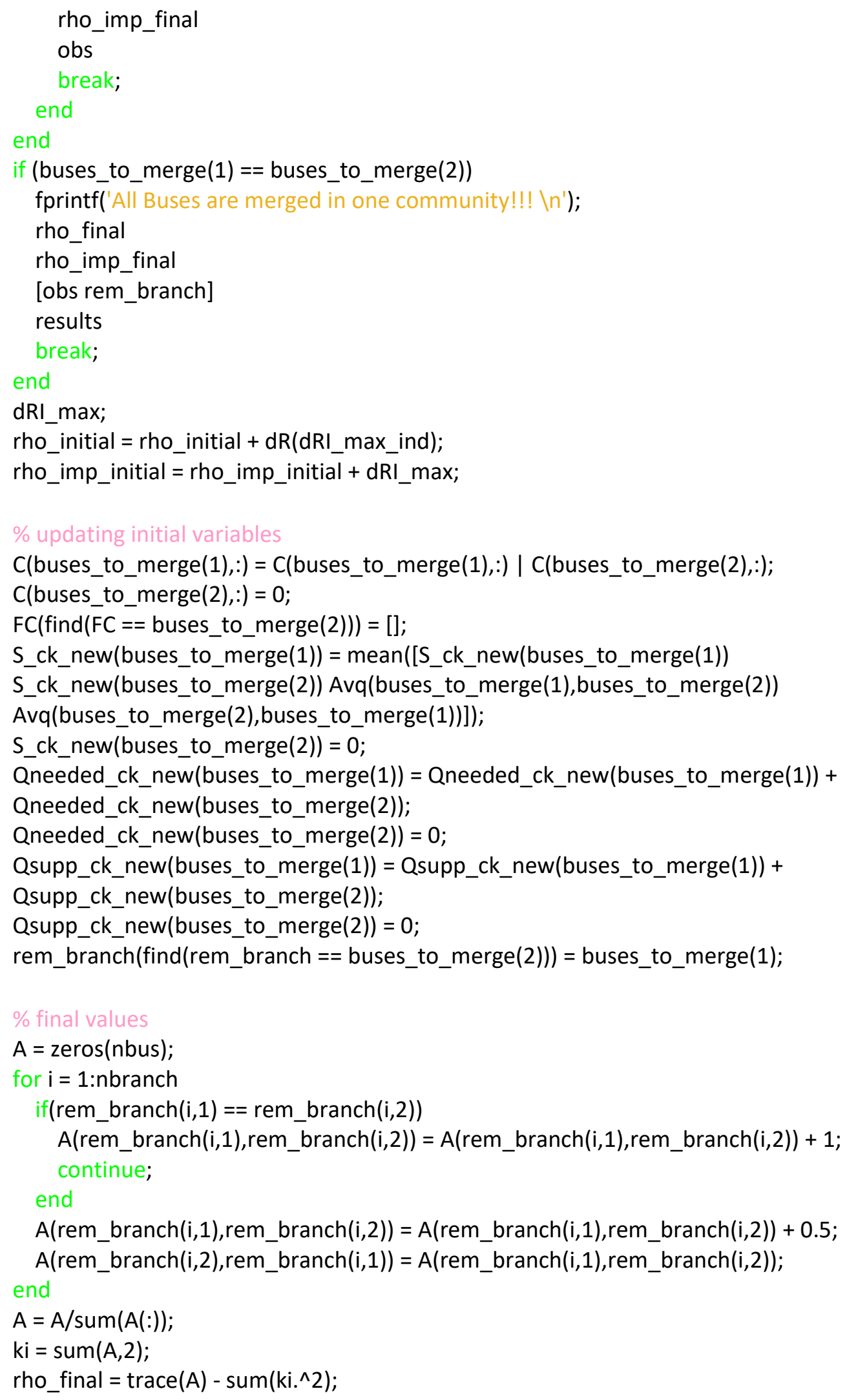




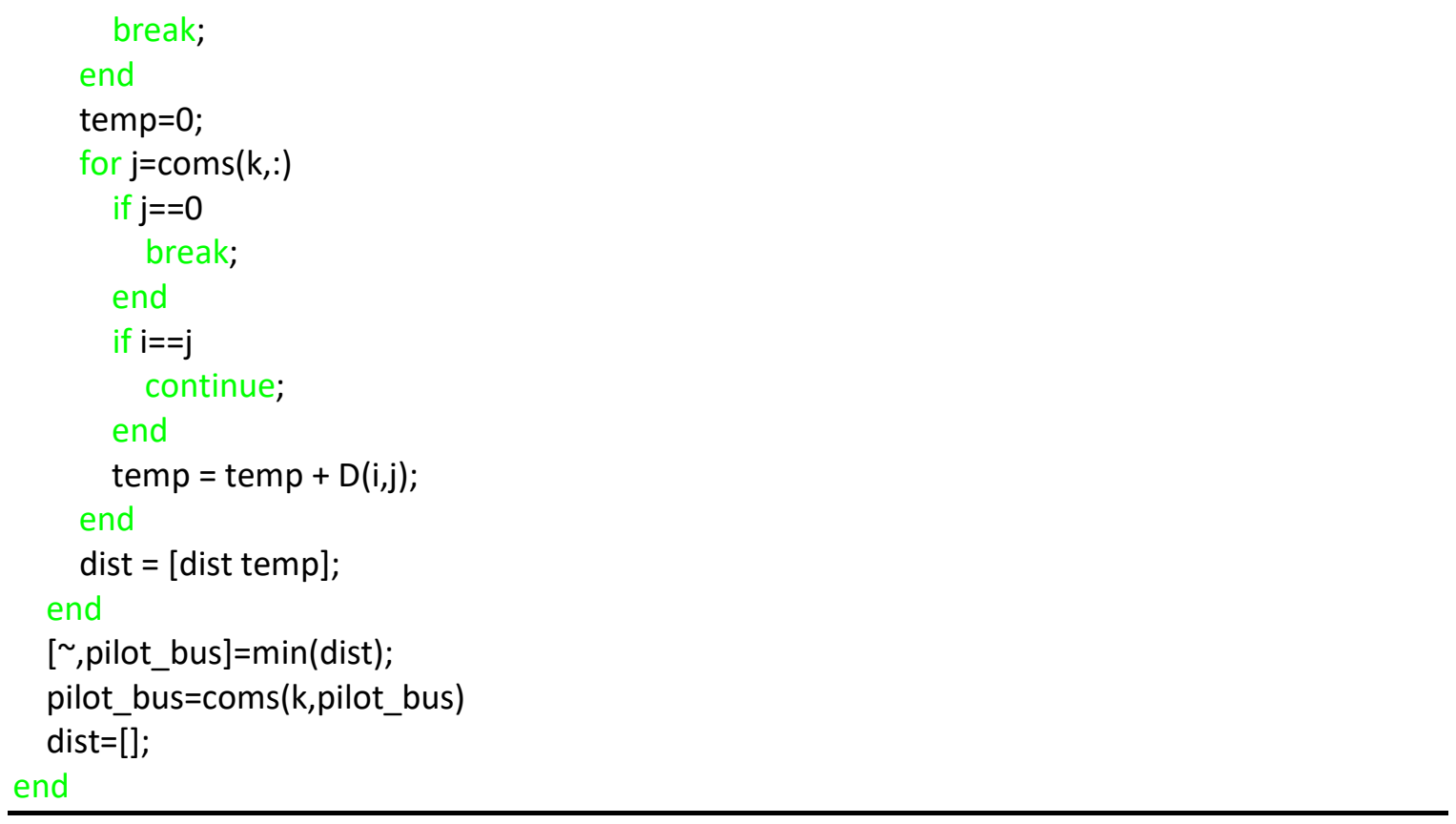

\section{SVC for 9-Bus Power System}

clc

clear

sys = case9;

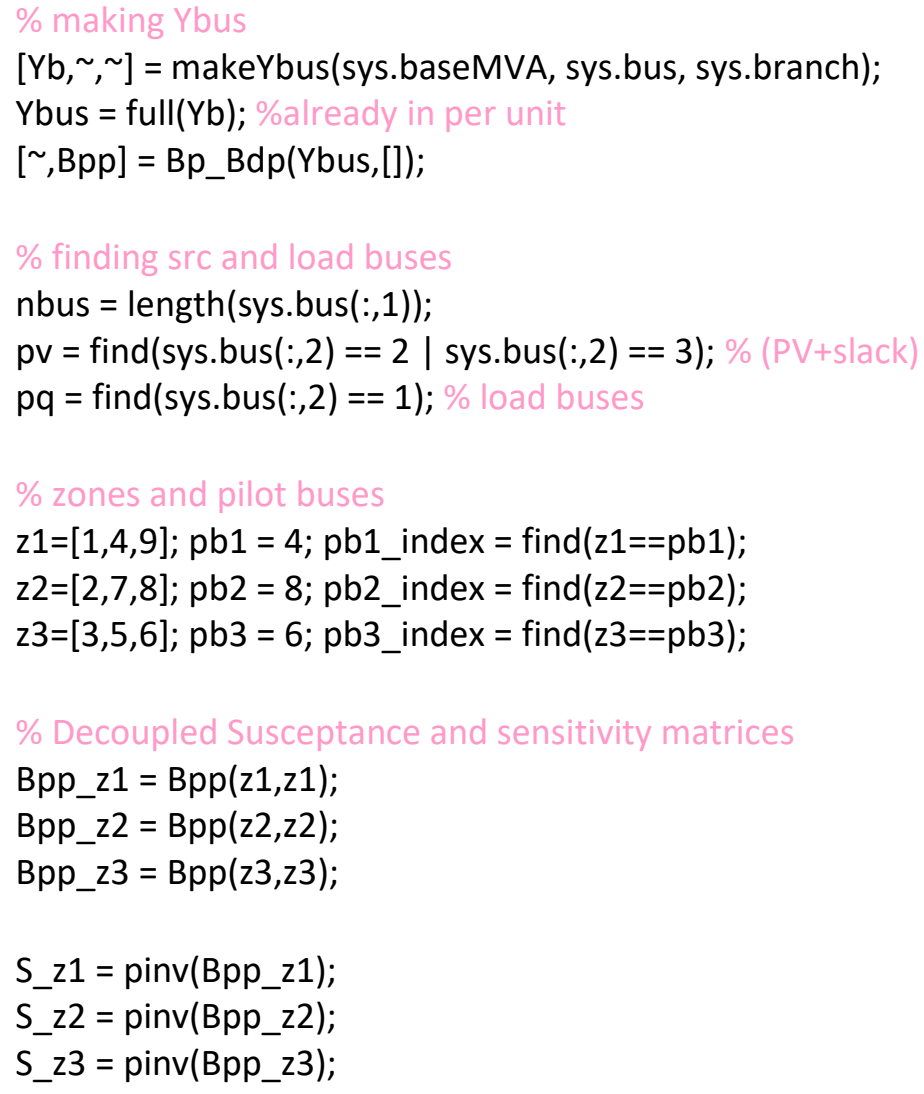




\section{APPENDIX. MATLAB PROGRAM FOR SVC}

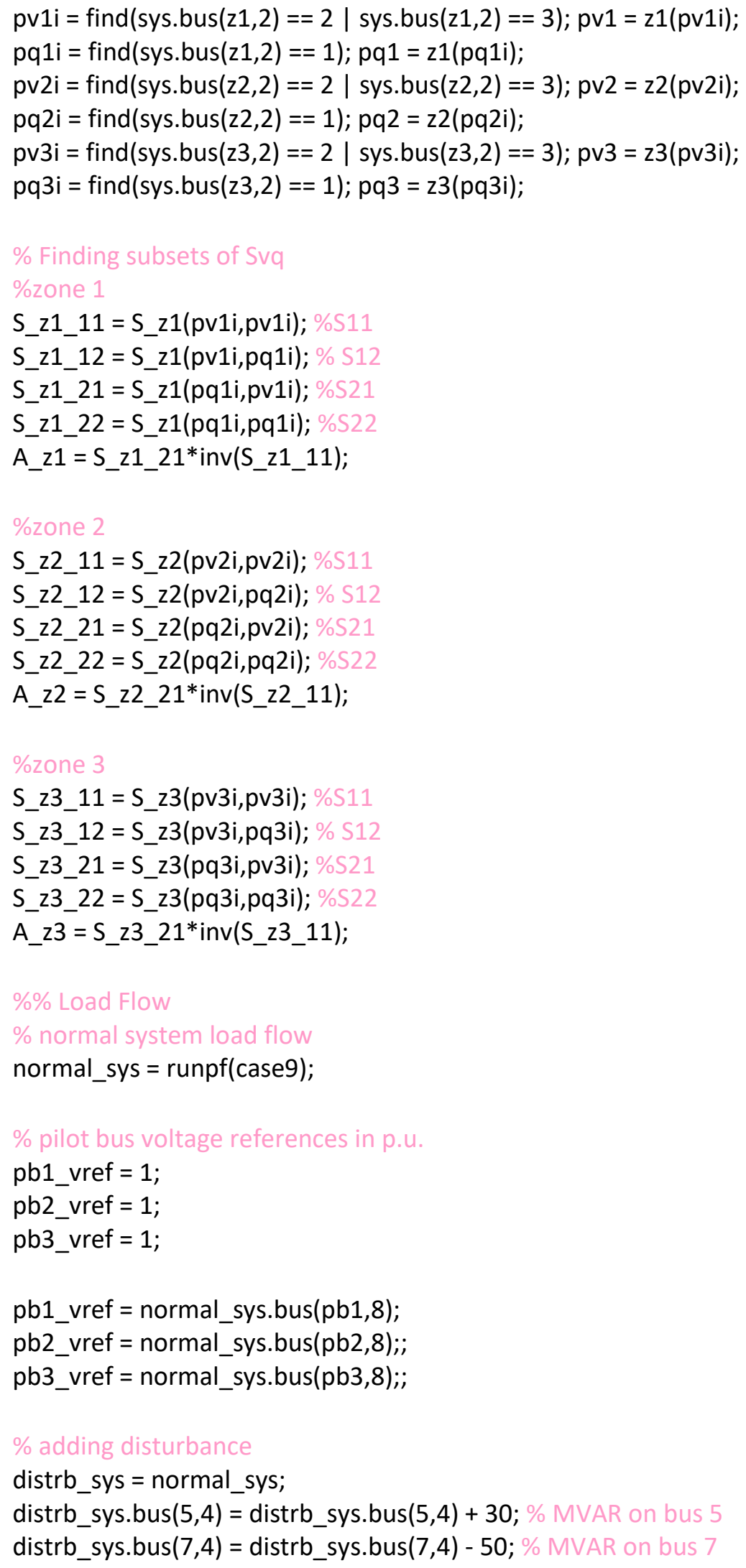

\section{$\% \%$ Load Flow}

$\%$ normal system load flow

normal_sys=runpf(case9);

$\%$ pilot bus voltage references in p.u.

pb1_vref $=1$;

pb2_vref = 1;

pb3_vref $=1$;

pb1_vref = normal_sys.bus $(p b 1,8)$;

pb2_vref $=$ normal_sys.bus $(\mathrm{pb} 2,8) ; ;$

pb3_vref = normal_sys.bus $(p b 3,8) ;$;

\section{$\%$ adding disturbance}

distrb_sys = normal_sys;

distrb_sys.bus $(5,4)=$ distrb_sys.bus $(5,4)+30 ; \%$ MVAR on bus 5

distrb_sys.bus $(7,4)=$ distrb_sys.bus $(7,4)-50 ; \%$ MVAR on bus 7 


\section{APPENDIX. MATLAB PROGRAM FOR SVC}

distrb_sys.bus $(9,4)=$ distrb_sys.bus $(9,4)+30 ; \%$ MVAR on bus 9

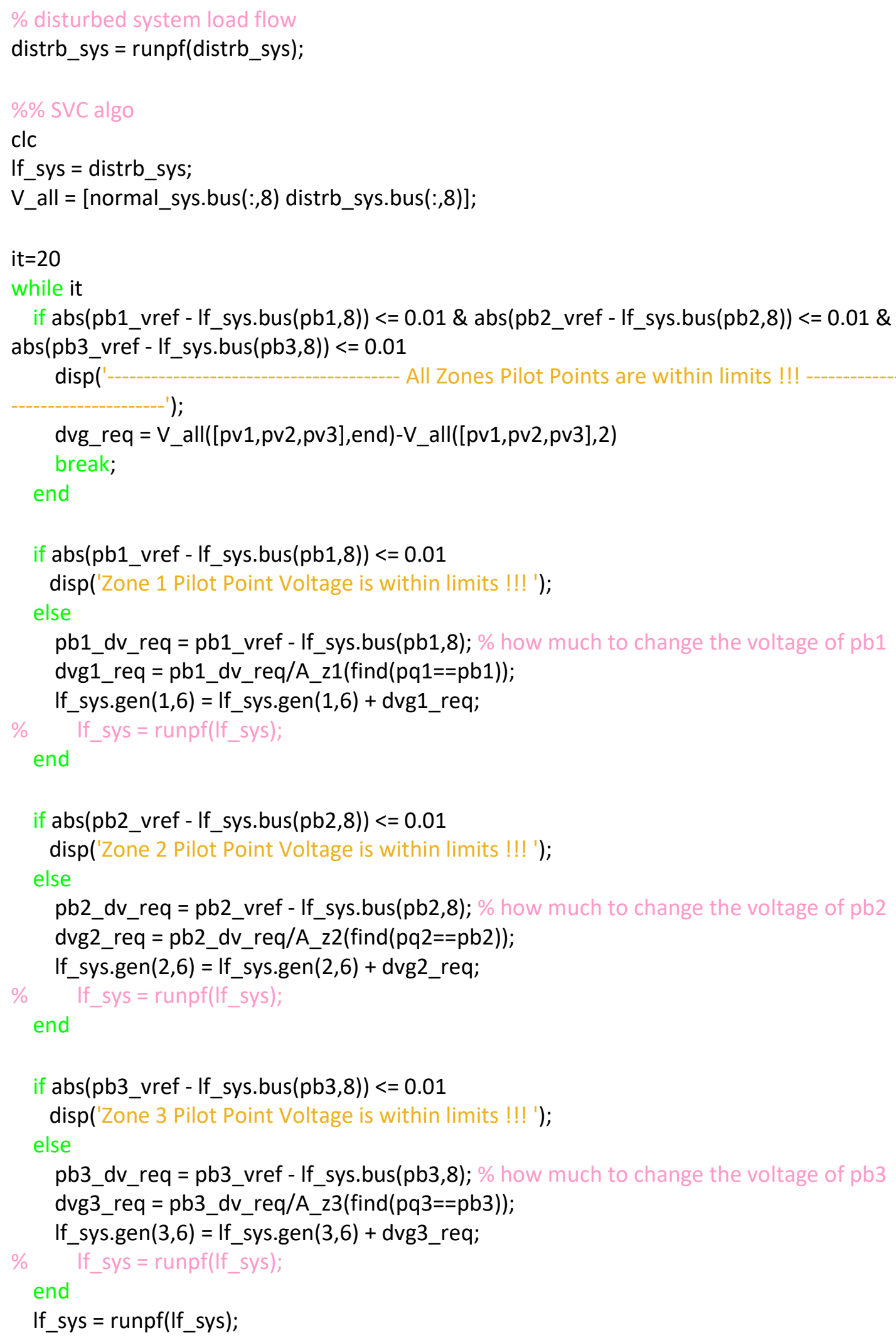




\title{
APPENDIX. MATLAB PROGRAM FOR SVC
}

\author{
it $=$ it -1 \\ V_all = [V_all If_sys.bus(:,8)]; \\ end \\ V_all \\ return
}

\section{SVC for 39-Bus Power System}

clc

clear

sys = case39;

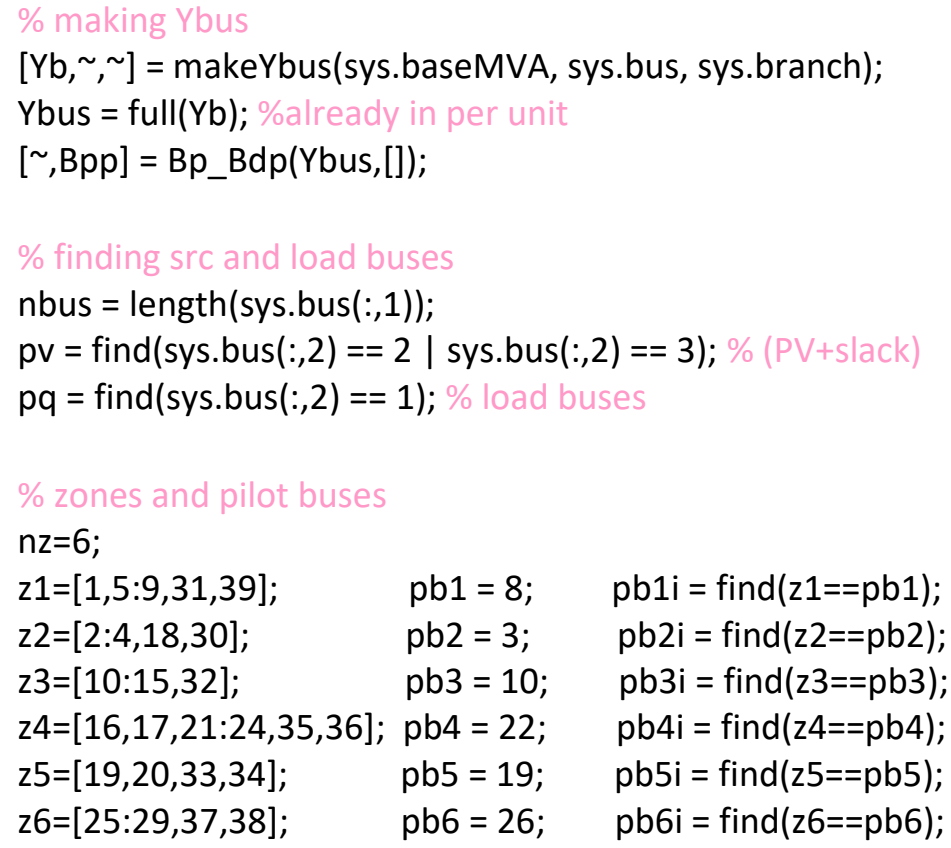




\section{APPENDIX. MATLAB PROGRAM FOR SVC}

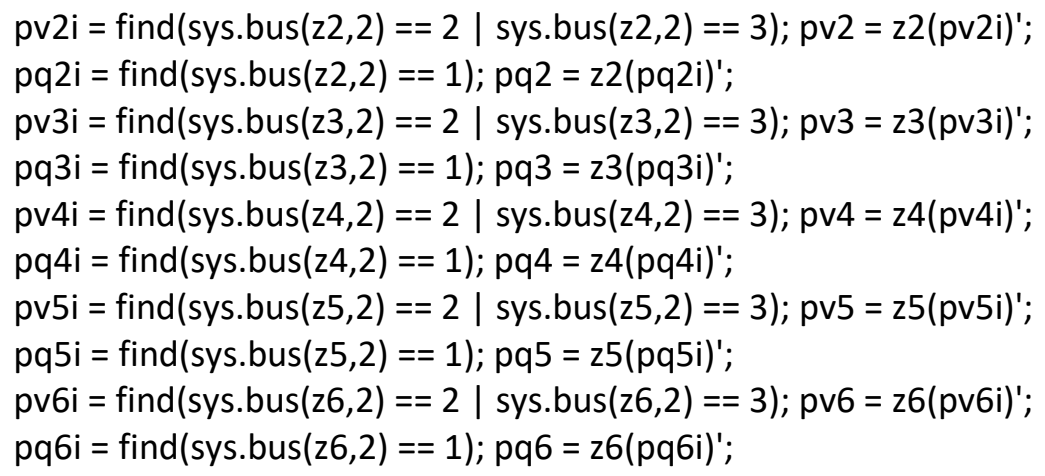

\section{\%zone 5}

S_z5_11 = S_z5(pv5i,pv5i);

S_z5_12 = S_z5(pv5i,pq5i); \% S12

S_z5_21 = S_z5(pq5i,pv5i);

S_z5_22 = S_z5(pq5i,pq5i); \%S22

A_z5 = S_z5_21*inv(S_z5_11); 


\section{APPENDIX. MATLAB PROGRAM FOR SVC}

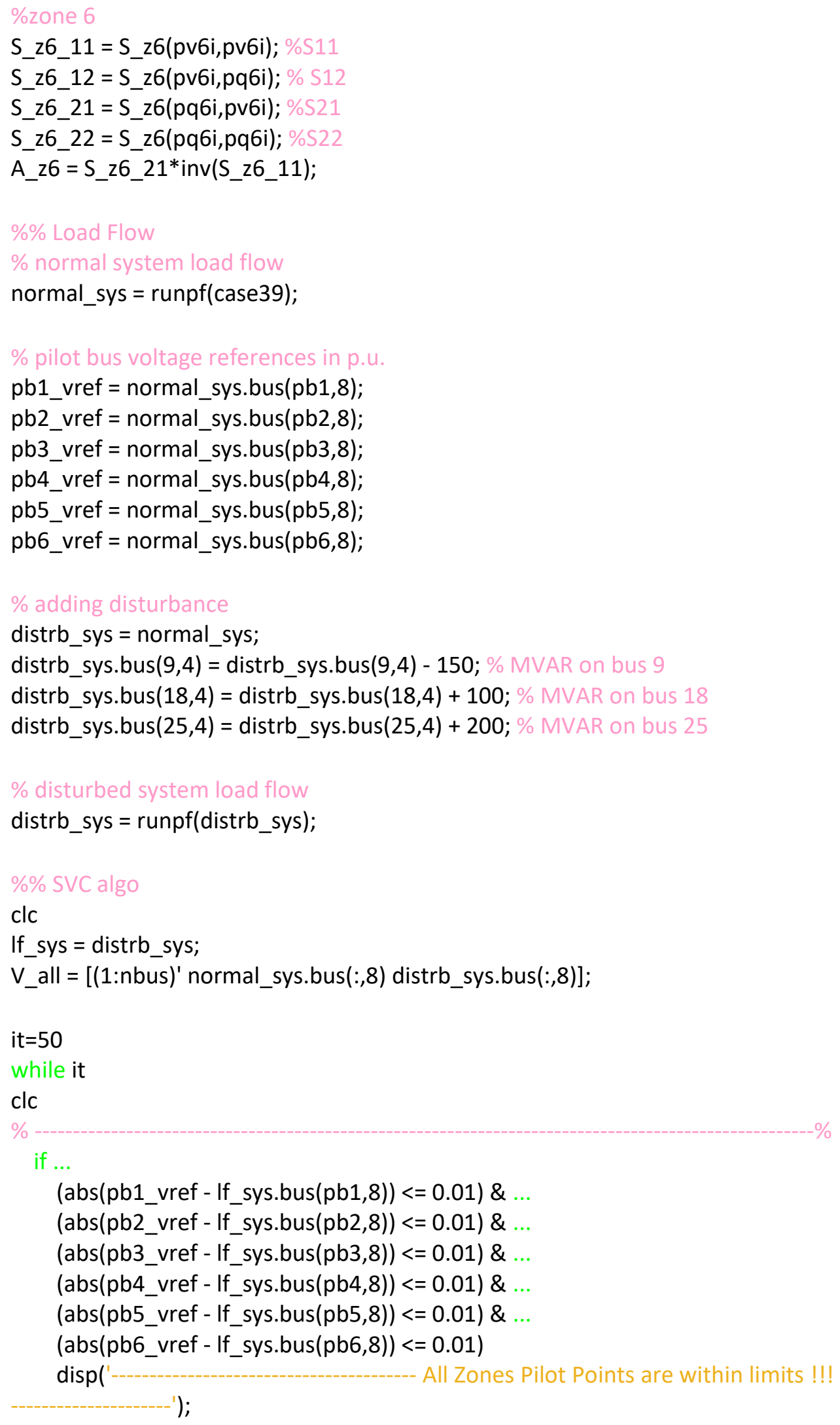




\section{APPENDIX. MATLAB PROGRAM FOR SVC}

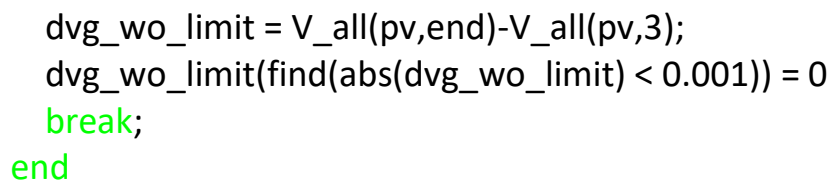




\section{APPENDIX. MATLAB PROGRAM FOR SVC}

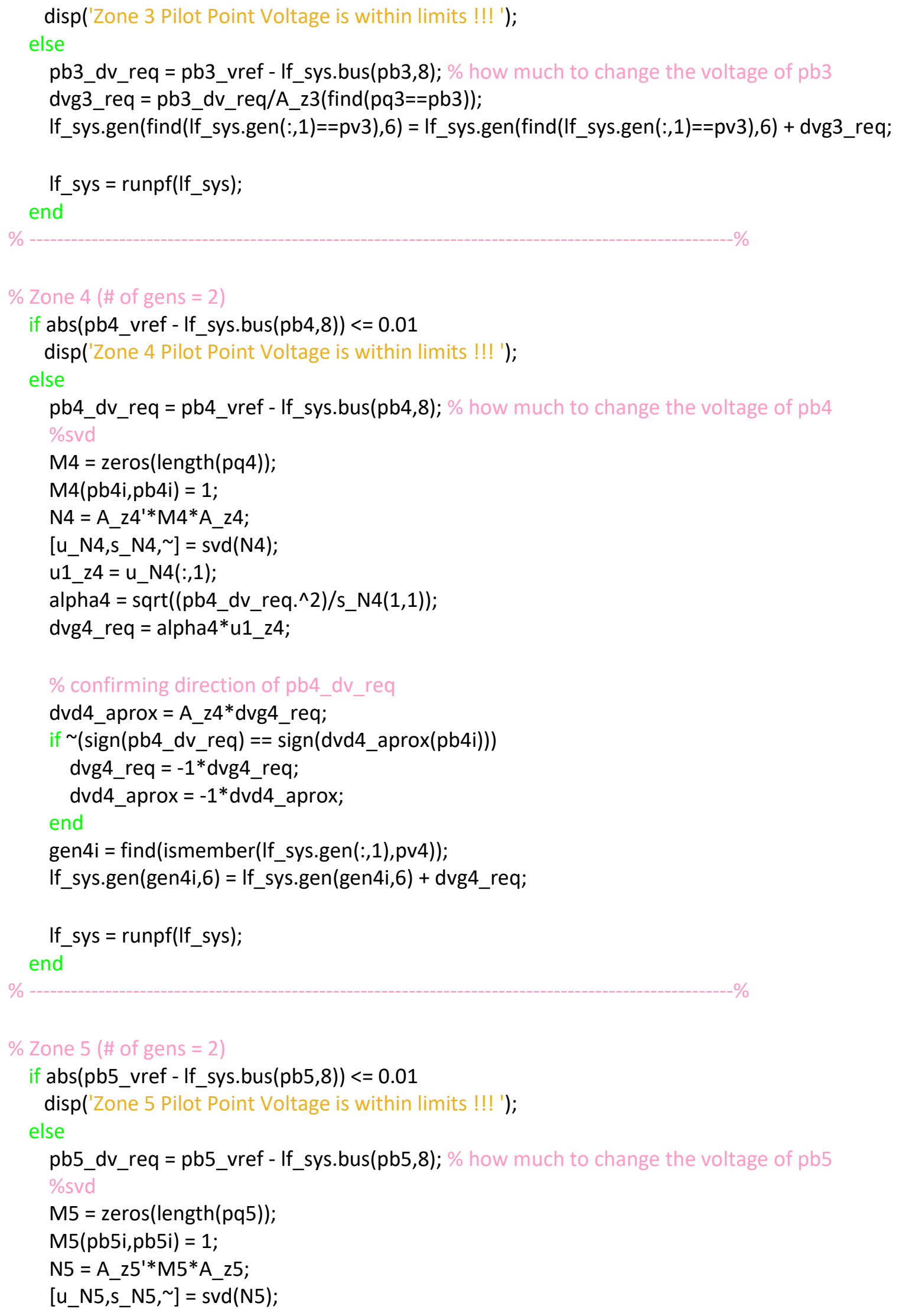




\section{APPENDIX. MATLAB PROGRAM FOR SVC}

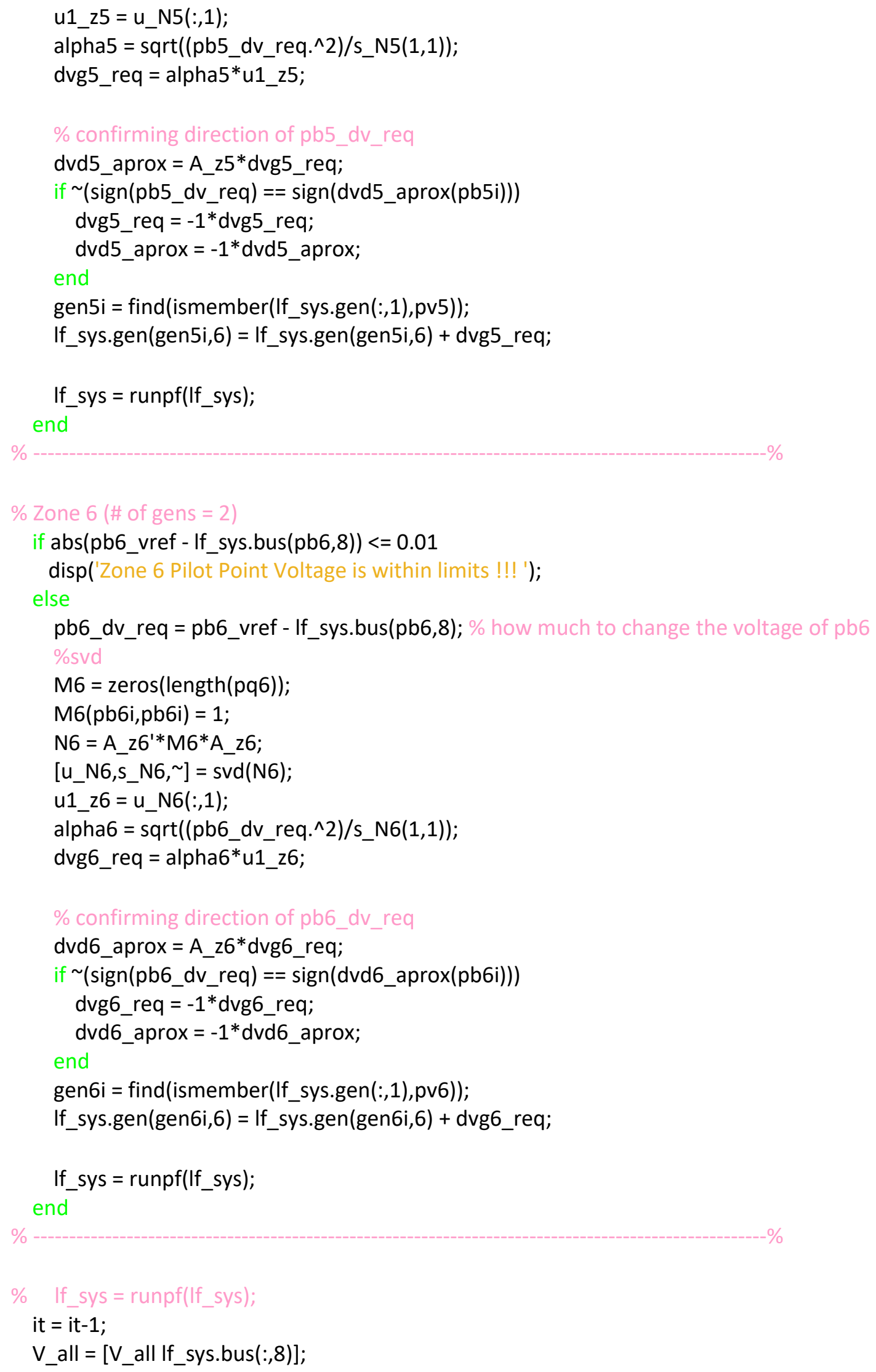




\title{
APPENDIX. MATLAB PROGRAM FOR SVC
}

\author{
V_all([pb1,pb2,pb3,pb4,pb5,pb6],:) \\ end \\ $\% \%$ max iteration check \\ if $(i t==0)$ \\ display('max. iter. reached. soln not converged'); \\ return \\ end
}

$\% \%$ after control

$\%$ pv_all = [pv1;pv2;pv3;pv4;pv5];

$\%\left[\sim\right.$,geni] = ismember $\left(p v \_\right.$all,distrb_sys.gen $\left.(:, 1)\right)$;

\section{$\% \mathrm{w} / \mathrm{o}$ limits}

distrb_sys_wo_limit = distrb_sys;

distrb_sys_wo_limit.gen(:,6) = distrb_sys_wo_limit.gen(:,6) + dvg_wo_limit;

distrb_sys_wo_limit $=$ runpf(distrb_sys_wo_limit);

V_all = [V_all distrb_sys_wo_limit.bus(:,8)];

\section{$\% \mathrm{w} /$ limits}

dvg_w_limit = dvg_wo_limit;

distrb_sys_w_limit $=$ distrb_sys;

lower_limit_viol = abs(distrb_sys_w_limit.gen(:,6)+ dvg_w_limit $)<0.9$;

Ilv_ind = find (lower_limit_viol ==1);

dvg_w_limit(llv_ind $)=0$;

distrb_sys_w_limit.gen $\left(I l v \_i n d, 6\right)=0.9$;

upper_limit_viol = abs(distrb_sys_w_limit.gen(:,6) + dvg_w_limit $)>1.1$;

ulv_ind = find(upper_limit_viol ==1);

dvg_w_limit(ulv_ind) =0;

distrb_sys_w_limit.gen $\left(u l v \_i n d, 6\right)=1.1$;

distrb_sys_w_limit.gen(:,6) = distrb_sys_w_limit.gen(:,6) + dvg_w_limit ;

distrb_sys_w_limit = runpf(distrb_sys_w_limit);

V_all = [V_all distrb_sys_w_limit.bus(:;8)];

dvg_w_limit $=$ V_all(pv,end) $-\mathbf{V} \_$all $(p v, 3) ; \%$ actual dvg after applying limits

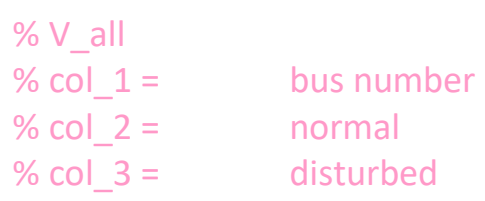


APPENDIX. MATLAB PROGRAM FOR SVC

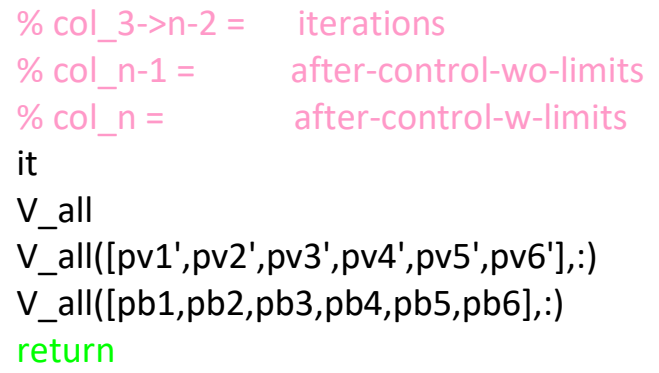




\section{References}

[1] S. Corsi, Voltage Control and Protection in Electrical Power Systems - From System Components to Wide Area Control. London, UK: Springer, 2015.

[2] B. M. Weedy, Electic Power Systems, 5th ed. New York: Wiley, 2012.

[3] C. W. Taylor, Power System Voltage Stability. New York: McGraw-Hill, 1994.

[4] F. Saccomanno, Electric Power Systems Analysis and Control. New York: WileyIEEE Press, 2003.

[5] P. Kundur, Power System Stability and Control. New York: McGraw-Hill, 1994.

[6] H. Saadat, Power System Analysis, 2nd ed. McGraw-Hill WCB, 2002.

[7] Y. Zhu et al., "Study on Reactive Power and Voltage Control in Large Grid Based on Sensitivity Analysis", Proc., Power and Energy Engineering Conference (APPEEC), IEEE 2012.

[8] R. Schlueter, I.-P. Hu, M. Chang, J. Lo, and A. Costi, "Methods for determining proximity to voltage collapse," IEEE Transactions on Power Systems, vol. 6, no. I, pp. 285-292, Feb 1991.

[9] R. Schlueter, "A voltage stability security assessment method," IEEE Transactions on Power Systems, vol. 13, no. 4, pp. 1423-1438, Nov 1998.

[10] C. Aumuller and T. Saha, "Determination of power system coherent bus groups by novel sensitivity-based method for voltage stability assessment," IEEE Transactions on Power Systems, vol. 18, no. 3, pp. 1157-1164, Aug 2003.

[11] P. Lagonotte, J.-c. Sabonnadiere, J. Leost, and J. Paul, "Structural analysis of the electrical system: application to secondary voltage control in france," IEEE Transactions on Power Systems, vol. 4, no. 2, pp. 479-486, May 1989.

[12] J. Zhong, E. Nobile, A. Bose, and K. Bhattacharya, "Localized reactive power markets using the concept of voltage control areas," IEEE Transactions on Power Systems, vol. 19, no. 3, pp. 1555-1561, Aug 2004.

[13] E. Nobile and A. Bose, "A new scheme for voltage control in a competitive ancillary service market," Proceedings of PSCC'02, 14th Power System Computation Conference, June 2002.

[14] R. Maharjan and S. Kamalasadan, "A New Approach for Voltage Control Area Identification Based on Reactive Power Sensitivities", North America Power Symposium (NAPS), 2015. 
[15] T. Iqbal, A. Feliachi, "Discovering Community Structures in Power System Networks using Voltage - Reactive Power Sensitivity," $49^{\text {th }}$ North American Power Symposium (NAPS), Sep. 2017.

[16] A. D. Banadaki, T. Iqbal, A. Feliachi, "Voltage Control using Singular Value Decomposition of Fast Decoupled Load Flow Jacobian," IEEE Power and Energy Society General Meeting (Poster), July 2017.

[17] T. Iqbal, A. D. Banadaki, A. Feliachi, "Optimal Voltage Control using Singular Value Decomposition of Fast Decoupled Load Flow Jacobian," 49 ${ }^{\text {th }}$ North American Power Symposium (NAPS), Sep. 2017.

[18] M. Moursi, G. Joos, C. Abbey, "A Secondary Voltage Control Strategy for Transmission Level Interconnection of Wind Generation," IEEE Transactions on Power Electronics, vol. 23, May 2008.

[19] B. M. Weedy, Electic Power Systems, 5th ed. New York: Wiley, 2012.

[20] O. A. Mousavi, R. Cherkaoui, "Literature Survey on Fundamental Issues of Voltage and Reactive Power Control," EPF Lausanne Power System Group, ETH, June 2011.

[21] Y. G. Rebours, D. S. Kirschen, "A Survey of Frequency and Voltage Control Ancillary Services-Part I: Technical Features," IEEE Transactions on Power Systems, Feb. 2007.

[22] J. L. Sancha, J. L. Fernandez, A. Cortes, J. T. Abarca, "Secondary Voltage Control: Analysis, Solutions and Simulation Results for the Spanish Transmission System," IEEE Transactions on Power Systems, vol. 11, no. 2, May 1996.

[23] N. A. Daher, I. Mougharbel, M. Saad, H. Y. Kanaan, "Comparative Study of Partitioning Methods Used for Secondary Voltage Control in Distributed Power Networks," IEEE International Conference on Smart Energy Grid Engineering (SEGE), 2013.

[24] P. O. L. Gatta, J. A. P. Filho, J. L. R. Pereira, "Comparison Among Methodologies for Identification of Pilot Buses and its Impact on the Steady State Secondary Voltage Control," 11th IEEE/IAS International Conference on Industry Applications, 2014.

[25] H. U. Banna, Z. Yu, D. Shi, Z. Wang, D. Su, C. Xu, S.K. Solanki, J. Solanki, "Online Coherence Identification Using Dynamic Time Warping for Controlled Islanding", Journal of Modern Power System and Clean Energy, under review [https://arxiv.org/abs/1708.06424v2]

[26] P. Lagonotte, J. Sabonnadiere, J. Leost, J. Paul, "Structural Analysis of the Electrical System: Application to Secondary Voltage Control in France," IEEE Transactions on Power Systems, vol. 4, pp. 479-486, May 1989.

[27] M. Shaaban, "Improved Regional Coordination of Generation Voltage Control," 2nd International Power and Energy Conference (PECon), pp. 175 - 179, December 2008. 
[28] J. Zhong, E. Nobile, A. Bose, "Localized Reactive Power Markets Using the Concept of Voltage Control Areas," IEEE Transactions on power systems, vol. 19, August 2004.

[29] S. Satsangi, A. Saini, A. Saraswat, "Voltage Control Areas for Reactive Power Management using Clustering Approach in Deregulated Power System," $2^{\text {nd }}$ International Conference on Sustainable Energy and Intelligent System (SEISCON 2011), July 2011.

[30] H. Mehrjerdi, S. Lefebvre, "Eliminating Voltage Violations in Power Systems using Secondary Voltage Control and Decentralized Neural Network," IEEE PES GM, 2013.

[31] J. A. Hartigan, M. A. Wong, "Algorithm AS 136: A k-means Clustering Algorithm," Applied statistics, pp. 100-108, 1979.

[32] G. Grigoras, B. Neagu, F. Scarlatache, R. C. Ciobanu, "Identification of Pilot Nodes for Secondary Voltage Control using K-means Clustering Algorithm," IEEE 26 ${ }^{\text {th }}$ International Symposium on Industrial Electronics (ISIE), 2017.

[33] J. Mezquita, D. Asber, S. Lefebvre, M. Saad, P. J. Lagacé, "Power Network Partitioning with a Fuzzy C-Means," Proceedings of the IASTED International Conference on Power and Energy Systems and Applications, PESA 2011, pp. 82-87, 2011.

[34] R. K. Pavão, "Evaluation of a Secondary Voltage Control Strategy using Selection of Pilot Bars and Control Areas through Fuzzy Logic," Pontífica Universidade Católica of Rio Grande do Sul. M.Sc. Dissertation. 2006.

[35] H. Mori, O. Matsuzaki, "A Rule-Based Tabu Search Technique for Power System Decomposition," IEEE Power Engineering Society Summer Meeting, vol.4, July 2000.

[36] S. Satsangi, A. Saini, A. Saraswat, "Voltage Control Areas for Reactive Power Management using Clustering Approach in Deregulated Power System," $2^{\text {nd }}$ International Conference on Sustainable Energy and Intelligent System (SEISCON 2011), July 2011.

[37] J. Mezquita, D. Asber, S. Lefebvre, M. Saad, "Power Network Partitioning with a Self-Organizing Map and Pilot Bus Selection with a Genetic Algorithm," GREPCI, 2012.

[38] S. Blumsack, P. Hines, M. Patel, C. Barrows, E. Cotilla Sanchez, "Defining Power Network Zones from Measures of Electrical Distance," IEEE PES GM, July 2009.

[39] B. Zhao, Z. Xu, C. Xu, "Network Partition Based Zonal Voltage Control for Distribution Networks with Distributed PV Systems," IEEE Transactions on Smart Grid, 2017.

[40] M. E. J. Newman, M. Girvan, "Finding and Evaluating Community Structures in Networks," Physics Review, 2004. 
[41] T. Iqbal, A. Feliachi, "Discovering Community Structures in Power System Networks using Voltage - Reactive Power Sensitivity," $49^{\text {th }}$ North American Power Symposium (NAPS), Sep. 2017.

[42] P. Lagonotte, J. C. Sabonnadiere, J. Y. Leost, and J. P. Paulm, "Structural Analysis of the Electrical System: Application to the Secondary Voltage Control in France," IEEE Transactions on Power Systems, May 1989.

[43] V. Arcidiacono, "Automatic Voltage and Reactive Power Control in Transmission Systems," IFAC Symposium on Power Systems, Florence, 1983.

[44] A. Conejo, T. Gbmez, J. I. de la Fuente, "Pilot- Bus Selection for Secondary Voltage Control," ETEP, vol. 3, no. 5, Sept/Oct. 1993.

[45] T. Amraee, A. Soroudi, and A.M. Ranjbar, "Probabilistic Determination of Pilot Points for Zonal Voltage Control," in IET Generation, Transmission and Distribution, 2012.

[46] L. Gatta, O. Paula, J. A. P. Filho, J. L. R. Pereira., "Comparison Among Methodologies for Identification of Pilot Buses and its Impact on the Steady State Secondary Voltage Control," in IEEE International Conference on Industry Applications, 2014.

[47] A. Conejo, J. I. De La Fuente. S. Goransson, "Comparison of Alternative Algorithms to Select Pilot Buses for Secondary Voltage Control in Electric Power Networks," Electrotechnical Conference 1994.

[48] A. D. Banadaki, T. Iqbal, A. Feliachi, "Voltage Control using Singular Value Decomposition of Fast Decoupled Load Flow Jacobian," IEEE Power and Energy Society General Meeting (Poster), July 2017.

[49] T. Iqbal, A. D. Banadaki, A. Feliachi, "Optimal Voltage Control using Singular Value Decomposition of Fast Decoupled Load Flow Jacobian," 49 $9^{\text {th }}$ North American Power Symposium (NAPS), Sep. 2017.

[50] H. U. Banna, D. Tiwari, S. K. Solanki, J. Solanki, "Load Variance Minimization using Coordinated PHEV Charging," 2016 North American Power Symposium, 1820 September 2016, Denver CO

[51] H. U. Banna, A. Luna, S. Ying, H. Ghorbani, P. Rodriguez, "Impacts of Wind Energy In-Feed on Power System Small Signal Stability," 3rd International Conference on Renewable Energy Research and Applications, Milwakuee, USA 1922 Oct 2014

[52] H. U. Banna, I. Faquir, A. Iqbal," Economic Analysis of a Small Hybrid Power System," IEEE Power Generation Systems and Renewable Energy Technologies, June10-11 2015, Islamabad, Pakistan

[53] H. U. Banna, A. Luna, P. Rodriguez, A. Cabrera, H. Ghorbani, S. Ying, "Performance Analysis of Conventional PSS and Fuzzy Controller for Damping Power System Oscillations," $3^{\text {rd }}$ International Conference on Renewable Energy Research and Applications, Milwakuee, USA 19-22 Oct 2014

[54] H. U. Banna, T. Iqbal, "Control System Design to Automate 100KV Impulse Generator," International Journal of Scientific and Engineering Research 2014 
[55] M. Girvan and M.E.J. Newman, "Community Structure in Social Biological Networks", Proc. Natl. Acad. Sci., Vol.99, No. 12, 2002.

[56] M.E.J. Newman and Girvan M, "Finding and Evaluating Community Structures in Networks", Physics Review, 2004.

[57] S. Gupta, R.M. Anderson, and R.M. May, "Networks of Sexual Contacts: Implications for the Pattern of Spread of HIV", AIDS 807, 1989.

[58] M.E.J. Newman, "Mixing Patterns in Networks", Physics Review, 2003.

[59] M.E.J. Newman, "Detecting Community Structures in Networks", The European Physical Journal B, Vol. 38, Issue 2, 2004.

[60] B. Zhao, Z. Xu, C. Xu, C. Wang and F. Lin, "Network Partition Based Zonal Voltage Control for Distribution Networks with Distributed PV Systems", IEEE Transactions on Smart Grid, 2017.

[61] M.E.J. Newman, "Fast Algorithm for Detecting Community Structure in Networks", Physics Review, 2004.

[62] B. Gao, G. K. Morison, P. Kundur, "Voltage Stability Evaluation using Modal Analysis", IEEE Transactions on power systems, vol. 7, no. 4, pp. 1529-1542, Nov 1992.

[63] P. Lagonotte, J. Sabonnadiere, J. Leost, and J. Paul, "Structural Analysis of the Electrical System: Application to Secondary Voltage Control in France", IEEE Transactions on power systems, vol. 4, pp. 479-486, May 1989.

[64] A. D. Nivine, M. Imad, "Pilot Buses Selection based on Reduced Jacobian Matrix", IEEE International Conference on Smart Energy Grid Engineering (SEGE), Aug 2015.

[65] R. D. Zimmerman, C. E. Murillo-Sánchez, and R. J. Thomas, "MATPOWER: Steady-State Operations, Planning and Analysis Tools for Power Systems Research and Education," Power Systems, IEEE Transactions on, vol. 26, no. 1, pp. 12-19, Feb. 2011.

[66] C. E. Murillo-Sánchez, R. D. Zimmerman, C. L. Anderson, and R. J. Thomas, "Secure Planning and Operations of Systems with Stochastic Sources, Energy Storage and Active Demand," Smart Grid, IEEE Transactions on, vol. 4, no. 4, pp. 2220-2229, Dec. 2013.

[67] T. Iqbal, A. Feliachi, "Discovering Community Structures in Power System Networks using Voltage - Reactive Power Sensitivity," 49 ${ }^{\text {th }}$ North American Power Symposium (NAPS), Sep. 2017.

[68] D. C. Lay, "Eigenvalues and Eigenvectors", in Linear Algebra and its Applications, 4th ed. Boston, MA: Pearson Education, 2012.

[69] G. Strang, "Eigenvalues and Eigenvectors", in Introduction to Linear Algebra, 5th ed. Welleslay MA: Welleslay-Cambridge Press, 2016.

[70] S. Tilekar. (2016, July 26). on physical significance of eigenvalues [Online], Available: $\quad$ https://www.quora.com/What-is-the-physical-significance-ofeigenvalues.

[71] D. C. Lay, "Symmetric Matrices and Quadratic Forms", in Linear Algebra and its Applications, 4th ed. Boston, MA: Pearson Education, 2012.

[72] G. Strang, "The Singular Value Decomposition (SVD)", in Introduction to Linear Algebra, 5th ed. Welleslay MA: Welleslay-Cambridge Press, 2016. 
[73] A. D. Banadaki, T. Iqbal, A. Feliachi, "Voltage Control using Singular Value Decomposition of Fast Decoupled Load Flow Jacobian," IEEE Power and Energy Society General Meeting (Poster), July 2017.

[74] T. Iqbal, A. D. Banadaki, A. Feliachi, "Optimal Voltage Control using Singular Value Decomposition of Fast Decoupled Load Flow Jacobian," 49th North American Power Symposium (NAPS), Sep. 2017.

[75] R. D. Zimmerman, C. E. Murillo-Sánchez, and R. J. Thomas, "MATPOWER: Steady-State Operations, Planning and Analysis Tools for Power Systems Research and Education," IEEE Transactions on Power Systems, vol. 26, no. 1, pp. 12-19, Feb. 2011.

[76] R. D. Zimmerman, C. E. Murillo-Sánchez, and R. J. Thomas, "MATPOWER's Extensible Optimal Power Flow Architecture," Power and Energy Society General Meeting, 2009 IEEE, pp. 1-7, July 26-30, 2009. 